\title{
Observable implications of geometrical and dynamical aspects of freeze-out in heavy ion collisions
}

\author{
Fabrice Retière ${ }^{1}$ and Michael Annan Lisa ${ }^{2}$ \\ ${ }^{1}$ Lawrence Berkeley National Laboratory, Berkeley, CA 94720 \\ ${ }^{2}$ Physics Department, The Ohio State University, Columbus, OH 43210
}

(Dated: November 2, 2018)

\begin{abstract}
Using an analytical parameterization of hadronic freeze-out in relativistic heavy ion collisions, we present a detailed study of the connections between features of the freeze-out configuration and physical observables. We focus especially on anisotropic freeze-out configurations (expected in general for collisions at finite impact parameter), azimuthally-sensitive HBT interferometry, and final-state interactions between non-identical particles. Model calculations are compared with data taken in the first year of running at RHIC; while not perfect, good agreement is found, raising the hope that a consistent understanding of the full freeze-out scenario at RHIC is possible, an important first step towards understanding the physics of the system prior to freeze-out.
\end{abstract}

PACS numbers: 25.75.Ld, 25.75.Gz, 24.10.Nz, 25.75.-q

\section{INTRODUCTION}

The first data from collisions between heavy nuclei at the Relativistic Heavy Ion Collider (RHIC) have generated intense theoretical efforts to understand the hot, dense matter generated in the early stage of the collision [1]. Testing these theoretical ideas relies on comparison to experimental observables. Leptonic [2] or electromagnetic [3] observables are believed to probe directly the early, dense stage of the collision. Most of the early data from RHIC, however, have been on hadronic observables. Measurements of hadrons at high transverse momentum $\left(p_{T}\right)$ [4] have generated much excitement, as they may provide useful probes of the dense medium produced at RHIC [5]. However, the medium itself decays largely into the soft $\left(\right.$ low- $\left.p_{T}\right)$ hadronic sector.

Soft hadronic observables measure directly the final "freeze-out" stage of the collision, when hadrons decouple from the bulk and free-stream to the detectors. Freeze-out may correspond to a complex configuration in the combined coordinate-momentum space, with collective components (often called "flow") generating spacemomentum correlations, as well as geometrical and dynamical (flow) anisotropies. A detailed experimentaldriven understanding of the freeze-out configuration is the crucial first step in understanding the system's prior evolution and the physics of hot colored matter.

In this paper, we explore in detail an analytic parameterization of the freeze-out configuration, which includes non-trivial correlations between coordinate- and momentum-space variables. We discuss the connections between the physical parameters of the model and observable quantities. If the model, with correct choice of physical model parameters, can adequately reproduce several independent measured quantities, then it might be claimed that this "crucial first step," mentioned above, has been performed.

A consistent reproduction of all low- $p_{T}$ observations at RHIC is not achieved in most physical models which aim to describe the evolution of the collision. In particular, it is difficult to reproduce momentum-space measurements while simultaneously describing the freezeout coordinate-space distribution probed by two-particle intensity interferometry measurements [6] (also known as Hanburry-Brown-Twiss or HBT 7] measurements). Hadronic cascade models predict a too weak momentum azimuthal anisotropy and too large source sizes [8]. Hydrodynamic transport models describe successfully transverse mass spectra and elliptic flow but fail at describing pion source radii [9]; some hydrodynamic models have successfully reproduced pion source radii [10], but only with different model parameters than those used to reproduce spectra and elliptic flow 11]. Similarly, sophisticated hybrid transport models (e.g. AMPT 12]) require different model parameters 13 to reproduce data on elliptic flow [15] and HBT [16]. Good reproduction of observed values has been acheived in models which adjust parameters to fit data within a given freeze-out scenario, such as in the Buda-Lund hydro approach [17]. The work presented here falls into this latter category.

The parameterization used in this paper ("blast-wave parameterization") is similar in form to the freezeout configuration obtained from hydrodynamic calculations [18], but we treat the physical parameters of the configuration (e.g. temperature) as free parameters. Our main goal is simply to quantify the driving physical parameters of freeze-out at RHIC, and the dependence of observables on these parameters.

Further motivation for exploring freeze-out configurations of the type discussed here, is that they implicitly assume a "bulk" system which may be described by global parameters (temperature, flow strength, etc). Discussions of a "new phase of matter" and its "Equation of State" are only sensible if indeed such assumptions hold. Comparison of blast-wave calculations with several independent measurements, then, is a crucial consistency check of these assumptions (though, of course, a successful comparison still would not constitute a proof of their validity). 
In transport models, whether the constituents are hadrons [19, 20], partons 21, 22], or fluid elements 9, 23, 24, 25], if they re-interact substantially, pressure gradients are generated, leading to collective velocity fields ("flow"), pushing the matter away from the hot center of the collision and into the surrounding vacuum. Evidence of collective flow, generated by final state re-interaction of collision products, has been based largely on interpretations of transverse mass spectra and transverse momentum azimuthal anisotropy 9]. However, this scenario has been challenged by new measurements of $\mathrm{p}$ - $\mathrm{Au}$ collisions [26] and new theoretical interpretations 27]. Indeed, so-called initial state effects such as random walk of the incoming nucleons 26] or Color Glass Condensate phenomena 27] may offer an alternative explanation of the measured spectra and anisotropies in transverse momentum. This ambiguity apparently threatens the concept that a bulk system has been created at all. However, it is important to recall that collective expansion, if it exists, would manifest itself not only in momentum-space observables, but would also generate space-momentum correlations, which can be measured via two-particle correlations.

The possible validity of any scenario may only be claimed if a single set of model parameters allows a successful description of all measured observables. Here, we study, in the context of a bulk collective flow scenario, transverse momentum spectra, momentum-space anisotropy ("elliptic flow"), HBT interferometry, and correlations between non-identical particles.

Similar studies have been reported previously [25, 28, 29, 30, 32]. New aspects in our study include: consideration of a more general (azimuthally-anisotropic) freezeout configuration, applicable to non-zero impact parameters; model studies of azimuthally-sensitive HBT interferometry and correlations between non-identical particles; and a multi-observable global fit to several pieces of published RHIC data.

This paper is organized as follows: In Section II, we describe the blast-wave parameterization. In Section III, we investigate in detail the sensitivity of several observables $\left(p_{T}\right.$ spectra, elliptic flow, pion HBT radii, and average space-time separation between different particle types) on the physical parameters of the blast-wave parameterization. In Section IV, we perform fits to published data measured at $\mathrm{RHIC}$ for $\mathrm{Au}+\mathrm{Au}$ collisions at $\sqrt{s_{N N}}=130 \mathrm{GeV}$, and, based on these fits, describe how as-yet unpublished analyses (azimuthally-sensitive HBT interferometry, and correlations between non-identical particles) are expected to look. The reader primarily interested in the quality of the fit to the data and the resulting parameters may want to skip past the details of Section III. In Section V, we summarize and conclude on the relevance of the blast-wave parameterization at RHIC.

\section{THE BLAST-WAVE PARAMETERIZATION}

\section{A. Geneology and Motivation}

More than a quarter of a century ago, Westfall et al 33 introduced the nuclear fireball model to explain midrapidity proton spectra. The idea was that the overlapping nucleons of the target and projectile combined to create a hot source with velocity between that of the target and projectile. Protons emitted from this source were expected to be emitted isotropically with a thermal energy distribution.

Soon thereafter, Bondorf, Garpman, and Zimanyi 34] derived a non-relativistic expression for the energy spectra of particles emitted from a thermal exploding source. The radial flow in their (spherical) source results in energy spectra increasingly different than those from a purely thermal (non-flowing) source, as the particle mass increases. Siemens and Rasmussen 35 then generalized the formula with relativistic kinematics, further simplifying by assuming a single expanding radial shell.

While a spherically-expanding source may be expected to approximate the fireball created in lower-energy collisions, at higher energies stronger longitudinal flow may lead to a cylindrical geometry. A decade ago, Schnedermann et al 36] introduced a simple functional form for the phasespace density at kinetic freezeout, which approximated hydrodynamical results assuming boostinvariant longitudinal flow [37], and successfully used it to fit $p_{T}$ spectra with only two parameters: a kinetic temperature, and a radial flow strength. The coordinate space geometry was an infinitely long solid cylinder (and so should approximate the situation for $b=0$ collisions at midrapidity); the transverse radial flow strength necessarily vanished along the central axis, and is assumed maximum at the radial edge. Most hydrodynamic calculations yield a transverse rapidity flow field linear in the radial coordinate 24 .

Huovinen et al [18] generalized this parameterization to account for the transversely anisotropic flow field which arises in non-central collisions, and which generates an elliptic flow signal similar to that seen in measurements 38]. This added one more parameter- the difference between the flow strength in, and out of, the reaction plane. The spatial geometry remained cylindrical, though it was assumed to be a cylindrical shell, not a solid cylinder.

The measured elliptical flow systematics as a function of $p_{T}$ and mass are fairly well-fit with the Huovinen parameterization [38]. However, better fits were achieved when the STAR Collaboration generalized the model even further, adding a fourth parameter designed to account for the anisotropic shape of the source in coordinate space [38]. A shell geometry was still assumed.

To calculate the spatial homogeneity lengths probed by two-particle correlation measurements [6], we must revert from the unrealistic shell geometry to a solid emission region (infinite series of elliptical shells). Furthermore, 
additional parameters corresponding to the source size, emission time, and emission duration must be included, increasing the number of parameters [42]. A similar generalization has been studied by Wiedemann [4]. Finally, in this paper, we explore the effects of a "hard-edge" versus a smooth spatial density profile; similar studies have recently been done by Tomášik et al [45] and Peitzmann [30] for the more restricted case of a transverselyisotropic source. This brings to eight the total number of parameters which we study.

Although the blast-wave functional form was motivated by its similarity to the freeze-out configuration of a real dynamical model (i.e. hydrodynamical solutions), it is not necessarily true that the hydrodynamical freezeout configuration corresponds to the parameter set that best describes the data. In this sense, the blast-wave model presented here remains only a parameterization. With eight freely-tunable parameters, it is clearly a toy model with little predictive power. However, the goal is to see whether a consistent description of the data from the soft sector at RHIC is possible within a simple boostinvariant model with transverse collective flow. If this turns out to be the case, then it is worthwhile considering that the parameter values indeed characterize the size, shape, timescales, temperature, and flow strengths of the freeze-out configuration. A consistent parameterization in terms of such physical quantities represents a true step forward, and provides valuable feedback to theorists constructing physical models of the collision.

\section{B. Parameters and Quantities in the blast-wave}

The eight parameters of the blast-wave parameterization described in this paper are $T, \rho_{0}, \rho_{2}, R_{y}, R_{x}, a_{s}, \tau_{0}$, and $\Delta \tau$; their physical meaning is given below.

The freeze-out distribution is infinite in the beam $(z)$ direction, and elliptical in the transverse $(x-y)$ plane. (The $x-z$ plane is the reaction plane.) The transverse shape is controlled by the radii $R_{y}$ and $R_{x}$, and the spatial weighting of source elements is given by

$$
\Omega\left(r, \phi_{s}\right)=\Omega(\tilde{r})=\frac{1}{1+e^{(\tilde{r}-1) / a_{s}}}
$$

where a fixed value of the "normalized elliptical radius"

$$
\tilde{r}\left(r, \phi_{s}\right) \equiv \sqrt{\frac{\left(r \cos \left(\phi_{s}\right)\right)^{2}}{R_{x}^{2}}+\frac{\left(r \sin \left(\phi_{s}\right)\right)^{2}}{R_{y}^{2}}}
$$

corresponds to a given elliptical sub-shell within the solid volume of the freeze-out distribution.

The parameter $a_{s}$ corresponds to a surface diffuseness of the emission source. As shown in Figure 11 a hard edge ("box profile") may be assumed by setting $a_{s}=0$, while the density profile approximates a Gaussian shape for $a_{s} \approx 0.3$.

It should be noted that the weighting function $\Omega\left(r, \phi_{s}\right)$ is not, in general, the source density distri-

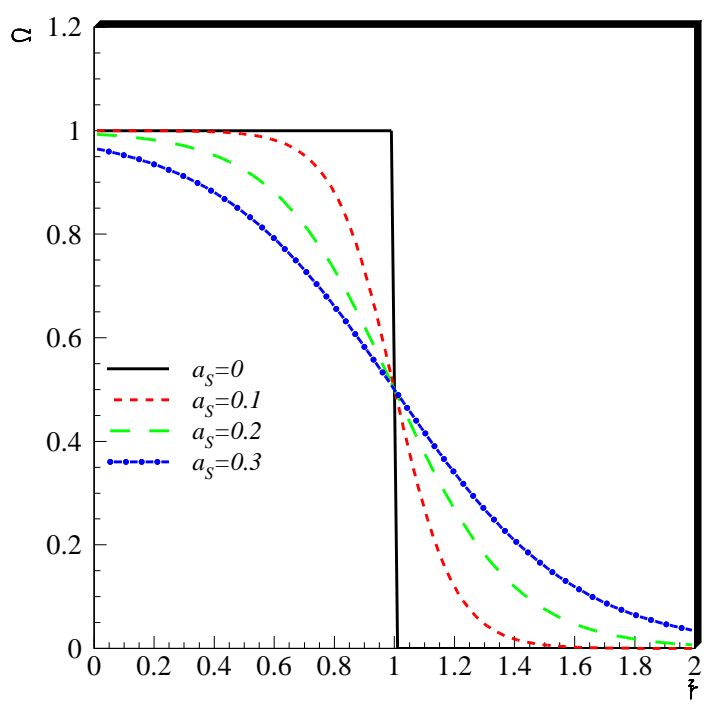

FIG. 1: (Color online) The source weighting function $\Omega$, as a function of the normalized elliptical radius $\tilde{r}$ for several values of the surface diffuseness parameter $a_{s}$.

bution. In particular, as we discuss especially in Sections IIC and IIID non-zero collective flow induces space-momentum correlations which dominate the spatial source density distributions. Only for a system without flow ( $\rho_{0}=\rho_{2}=0$, see below), the source distribution is given by $\Omega$, so that, e.g., for $a_{s}=0$, there is a uniform density of sources $\left(\frac{d^{2} N}{d x d y}=\right.$ const $)$ inside the ellipse defined by $R_{y}$ and $R_{x}$, and no sources outside.

The momentum spectrum of particles emitted from a source element at $(x, y, z)$ is given by a fixed temperature $T$ describing the thermal kinetic motion, boosted by a transverse rapidity $\rho(x, y)$. This is common in models of this type. However, unlike transversely isotropic parameterizations, the azimuthal direction of the boost (denoted $\left.\phi_{b}\right)$ is not necessarily identical to the spatial azimuthal angle $\phi_{s}=\tan ^{-1}(y / x)$. Instead, in our model, the boost is perpendicular to the elliptical sub-shell on which the source element is found; see Figure 2 We believe this to be a more natural extension of an "outward" boost for non-isotropic source distributions than that used by Heinz and Wong [31], who used an anisotropic shape but always assumed radial boost direction $\left(\phi_{b}=\phi_{s}\right)$. It may be shown that for our model

$$
\tan \left(\phi_{s}\right)=\left(\frac{R_{y}}{R_{x}}\right)^{2} \tan \left(\phi_{b}\right)
$$

Hydrodynamical calculations for central collisions (i.e. azimuthally isotropic freezeout distribution) suggest that the flow rapidity boost depends linearly on the freeze-out radius 24]. We assume a similar scenario, but in our more generalized parameterization, the boost strength depends linearly on the normalized elliptical radius $\tilde{r}$ de- 


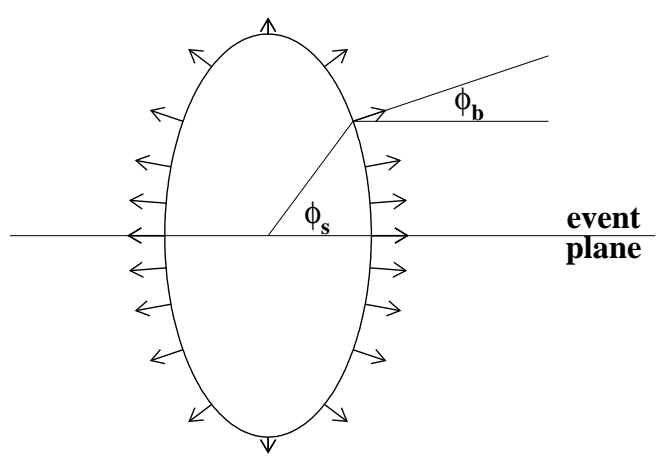

FIG. 2: Schematic illustration of an elliptical sub-shell of the source. Here, the source is extended out of the reaction plane $\left(R_{y}>R_{x}\right)$. Arrows represent the direction and magnitude of the flow boost. In this example, $\rho_{2}>0$ (see Equation 4 ).

fined in Equation 2] Thus, in the absence of an azimuthal dependence of the flow (to be introduced shortly), all source elements on the outer edge of the source boost with the same (maximum) transverse rapidity $\rho_{0}$ in an "outward" direction.

In non-central collisions, the strength of the flow boost itself may depend on azimuthal angle, as suggested by Huovinen et al [18]. As those authors did, we incorporate this via a parameter $\rho_{2}$, which characterizes the strength of the second-order oscillation of the transverse rapidity as a function of $\phi_{b}$. Hence, the flow rapidity is given by

$$
\rho\left(r, \phi_{s}\right)=\tilde{r}\left(\rho_{0}+\rho_{2} \cos \left(2 \phi_{b}\right)\right) .
$$

We note that source anisotropy enters into our parameterization in two independent ways, and each contributes to, e.g., elliptic flow. Setting $\rho_{2}>0$ means the boost is stronger in-plane than out-of-plane, contributing to positive elliptic flow. However, even if $\rho_{2}=0$ (but $\rho_{0} \neq 0$ ), setting $R_{y}>R_{x}$ still generates positive elliptic flow, since this means there are more sources emitting in-plane than out-of-plane (see Figure 2). The STAR Collaboration found that both types of anisotropy were required to fit their elliptic flow data [38]. In generalizing the circular transverse geometry parameterization of Huovinen et al [18] (in which $\phi_{b}=\phi_{s}$ ), they added a parameter $s_{2}$ and weighted source elements with a given $\phi_{b}$ as

$$
\frac{d N}{d \phi_{b}}=\frac{d N}{d \phi_{s}} \sim\left(1+2 s_{2} \cos \left(2 \phi_{s}\right)\right)
$$

Thus, a positive value of $s_{2}=\left\langle\cos \left(2 \phi_{b}\right)\right\rangle$ corresponded to more source elements emitting in-plane, similar to setting $R_{y}>R_{x}$ in our parameterization.

To facilitate comparison of fits with the STAR model and with ours, we relate the $s_{2}$ of STAR to the geometric anisotropy of our parameterization. In the case of isotropic boost $\left(\rho_{2}=0\right)$ [6],

$$
s_{2}=\left\langle\cos \left(2 \phi_{b}\right)\right\rangle=\frac{1}{2} \frac{\left(\frac{R_{y}}{R_{x}}\right)^{2}-1}{\left(\frac{R_{y}}{R_{x}}\right)^{2}+1} .
$$

If $\rho_{2} \neq 0$, anisotropies in the space-momentum correlations lead to a significantly more complicated expression.

Finally, since our model is based on a longitudinally boost-invariant assumption, it is sensible that the freezeout occurs with a given distribution in longitudinal proper time $\tau=\sqrt{t^{2}-z^{2}}$. We assume a Gaussian distribution peaked at $\tau_{0}$ and with a width $\Delta \tau$

$$
\frac{d N}{d \tau} \sim \exp \left(-\frac{\left(\tau-\tau_{0}\right)^{2}}{2 \Delta \tau^{2}}\right)
$$

We note that although the source emits particles over a finite duration in proper time $\tau$, we assume that none of the source parameters changes with $\tau$. This is obviously an oversimplification valid only for small $\Delta \tau$; with time, one may expect the flow field to evolve (increase or decrease), and it is natural to expect the transverse sizes $R_{x}$ and $R_{y}$ to change (grow or fall) with time. However, calculation of the time dependence of these parameters requires a true dynamical model and is outside the scope and spirit of the present work.

\section{The Emission Function}

Our emission function is essentially a generalization of azimuthally-isotropic emission functions used by previous authors [53, 55, 56, 57], and here we follow closely [55]:

$$
\begin{aligned}
S(x, K)= & m_{T} \cosh (\eta-Y) \Omega\left(r, \phi_{s}\right) e^{\frac{-\left(\tau-\tau_{0}\right)^{2}}{2 \Delta \tau^{2}}} \times \\
& \frac{1}{e^{K \cdot u / T} \pm 1} \\
= & m_{T} \cosh (\eta-Y) \Omega\left(r, \phi_{s}\right) e^{\frac{-\left(\tau-\tau_{0}\right)^{2}}{2 \Delta \tau^{2}}} \times \\
& \sum_{n=1}^{\infty}(\mp 1)^{n+1} e^{-n K \cdot u / T} .
\end{aligned}
$$

Where the upper (lower) sign is for fermions (bosons). Often, only the first term in the sum in Equation 8 is used, resulting in a Boltzmann distribution for all particles. Below, we show that there is a small change to observables when truncating after the second term, and negligible effect when including further terms. The Boltzmann factor $\exp (-K \cdot u / T)$ arises from our assumption of local thermal equilibrium within a source moving with four-velocity $u_{\mu}(x)$. We assume longitudinal boost invariance by setting the longitudinal flow velocity $v_{L}=z / t(z=\tau \sinh \eta$ and $t=$ $\tau \cosh \eta)$, so that the longitudinal flow rapidity $\eta_{\text {flow }}=$ $\frac{1}{2} \ln \left[\left(1+v_{L}\right) /\left(1-v_{L}\right)\right]$ is identical 37] to the space-time 
rapidity $\eta=\frac{1}{2} \ln [(t+z) /(t-z)]$. Thus, in cylindrical coordinates

$$
\begin{array}{r}
u_{\mu}(x)=\left(\cosh \eta \cosh \rho\left(r, \phi_{s}\right), \sinh \rho\left(r, \phi_{s}\right) \cos \phi_{b},\right. \\
\left.\sinh \rho\left(r, \phi_{s}\right) \sin \phi_{b}, \sinh \eta \cosh \rho\left(r, \phi_{s}\right)\right)
\end{array}
$$

and

$$
K_{\mu}=\left(m_{T} \cosh Y, p_{T} \cos \phi_{p}, p_{T} \sin \phi_{p}, m_{T} \sinh Y\right),
$$

where the transverse momentum $\left(p_{T}\right)$, transverse mass $\left(m_{T}\right)$, rapidity $(Y)$, and azimuthal angle $\left(\phi_{p}\right)$ refer to the momentum of the emitted particle, not the source element. (Note that three azimuthal angles- $\phi_{s}, \phi_{b}$, and $\phi_{p}-$ are relevant to this discussion.) Thus

$$
\begin{array}{r}
K_{\mu} u^{\mu}=m_{T} \cosh \rho\left(r, \phi_{s}\right) \cosh (\eta-Y)- \\
p_{T} \sinh \rho\left(r, \phi_{s}\right) \cos \left(\phi_{b}-\phi_{p}\right),
\end{array}
$$

and the emission function (Equation 8) may be rewritten as

$$
\begin{aligned}
S(x, K)= & S\left(r, \phi_{s}, \tau, \eta\right) \\
= & m_{T} \cosh (\eta-Y) \Omega\left(r, \phi_{s}\right) e^{\frac{-\left(\tau-\tau_{0}\right)^{2}}{2 \Delta \tau^{2}}} \times \\
& \sum_{n=1}^{\infty}(\mp 1)^{n+1} e^{n \alpha \cos \left(\phi_{b}-\phi_{p}\right)} e^{-n \beta \cosh (\eta-Y)} .
\end{aligned}
$$

where we define

$$
\begin{array}{r}
\alpha \equiv \frac{p_{T}}{T} \sinh \rho\left(r, \phi_{s}\right) \\
\beta \equiv \frac{m_{T}}{T} \cosh \rho\left(r, \phi_{s}\right) .
\end{array}
$$

Exploiting the boost invariance and infinite longitudinal extension of our source, and focusing on observables at midrapidity and using the longitudinally co-moving system (LCMS) for HBT measurements, we may simplify Equation 12 by setting $Y=0$.

\section{Calculating Observables}

All observables which we will calculate are related to integrals of the emission function (12) over phasespace $d^{4} x=d x d y d z d t=\tau d \tau d \eta r d r d \phi_{s}$, weighted with some quantity $B(x, K)$. In all cases, the integrals over $\tau$ and $\eta$ may be done analytically, though the result depends on whether $B(x, K)$ itself depends on $\tau$ and $\eta$.

In particular, if $B(x, K)=$ $B^{\prime}\left(r, \phi_{s}, K\right) \tau^{i} \sinh ^{j} \eta \cosh ^{k} \eta$ then the integrals of interest are [58]

$$
\begin{gathered}
\int_{0}^{2 \pi} d \phi_{s} \int_{0}^{\infty} r d r \int_{-\infty}^{\infty} d \eta \int_{-\infty}^{\infty} \tau d \tau S(x, K) B(x, K) \\
=m_{T} H_{i} \cdot\left\{B^{\prime}\right\}_{j, k}(K)
\end{gathered}
$$

where the $\tau$ and $\eta$ integrals are denoted

$$
\begin{aligned}
& H_{i} \equiv \int_{-\infty}^{\infty} d \tau \tau^{i+1} e^{\frac{-\left(\tau-\tau_{0}\right)^{2}}{2 \Delta \tau^{2}}} \\
& H_{0}=\sqrt{2 \pi} \cdot \Delta \tau \cdot \tau_{0} \\
& H_{1}=\sqrt{2 \pi} \cdot \Delta \tau \cdot\left(\Delta \tau^{2}+\tau_{0}^{2}\right) \\
& H_{2}=\sqrt{2 \pi} \cdot \Delta \tau \cdot \tau_{0}\left(3 \Delta \tau^{2}+\tau_{0}^{2}\right)
\end{aligned}
$$

and

$$
\begin{aligned}
G_{j, k}(x, K) & \equiv \int_{-\infty}^{\infty} d \eta e^{-\beta \cosh \eta} \sinh ^{j} \eta \cosh ^{k+1} \eta \\
G_{0,0}(x, K) & =2 \mathrm{~K}_{1}(\beta) \\
G_{0,1}(x, K) & =2\left[\frac{\mathrm{K}_{1}(\beta)}{\beta}+\mathrm{K}_{0}(\beta)\right] \\
G_{1,0}(x, K) & =G_{1,1}(x, K)=0 \\
G_{0,2}(x, K) & =2\left[\frac{\mathrm{K}_{2}(\beta)}{\beta}+\mathrm{K}_{1}(\beta)\right] \\
G_{2,0}(x, K) & =2 \frac{\mathrm{K}_{2}(\beta)}{\beta} .
\end{aligned}
$$

$\beta$ was defined in (14), and $K_{n}$ are the modified Bessel functions. For the above, we define the notation

$$
\begin{aligned}
& \left\{B^{\prime}\right\}_{j, k}(K) \equiv \sum_{n=1}^{\infty}\left\{(\mp 1)^{n+1} \int_{0}^{2 \pi} d \phi_{s} \int_{0}^{\infty} r d r[\right. \\
& \left.\left.G_{j, k}(x, n K) B^{\prime}(x, K) \times e^{n \alpha \cos \left(\phi_{b}-\phi_{p}\right)} \Omega\left(r, \phi_{s}\right)\right]\right\}
\end{aligned}
$$

for the remaining integrals, which we perform numerically. (Note that $G_{i, j}(x, n K)$ retains dependence on $r$ and $\phi_{s}$ due to its dependence on $\beta$, as defined in Equation 14 and so cannot move outside the integrals in Equation 18)

\section{CALCULATION OF HADRONIC OBSERVABLES}

In this Section, we discuss how hadronic observables are calculated from the parameterized source and illustrate the sensitivity of these observables to the various parameters presented in Section IB

With several observables depending on several parameters, it is not feasible to explore the entire numerical parameter space. Instead, we anticipate the results of the next Section, in which we fit our model to existing data, and vary the parameters by "reasonable" amounts about values similar to those which fit the data. The default parameter values used in several of the calculations in this Section are listed in Table \ 


\begin{tabular}{|c|c|c|}
\hline parameter & round source & non-round source \\
\hline$\rho_{2}$ & 0 & 0.05 \\
\hline$R_{x}(\mathrm{fm})$ & 12.04 & 11 \\
\hline$R_{y}(\mathrm{fm})$ & 12.04 & 13 \\
\hline$T(\mathrm{GeV})$ & & 0.1 \\
\hline$\rho_{0}$ & & 0.9 \\
\hline$\tau_{0}(\mathrm{fm} / \mathrm{c})$ & & 9 \\
\hline$\Delta \tau(\mathrm{fm} / \mathrm{c})$ & & 2 \\
\hline$a_{s}$ & & 0 \\
\hline
\end{tabular}

TABLE I: Default parameter values for most calculations in Section III Note that $\rho_{2}, R_{x}$ and $R_{y}$ default values depend on whether we are discussing an azimuthally isotropic ("round") or anisotropic ("non-round") source. One might expect such sources from central and peripheral collisions, respectively.

\section{A. $p_{T}$ spectra}

In the notation of Section IID the (azimuthallyintegrated) $p_{T}$ spectrum is calculated as

$$
\begin{aligned}
\frac{d N}{p_{T} d p_{T}} & =\int d \phi_{p} \int d^{4} x S(x, K) \\
& \propto m_{T} \int d \phi_{p}\{1\}_{0,0}(K) .
\end{aligned}
$$

In this paper, we focus only on the shapes, not the normalizations, of the spectra.

We note that spectra calculated in the blast-wave model scale neither with $m_{T}$ nor $p_{T}$, as both quantities enter the expression through $\alpha$ and $\beta$ (Equations (13) and (14)). This breaking of $m_{T}$-scaling is a well-known consequence of finite transverse flow $[36](\rho \neq 0$ in our model).

According to Equation $19 m_{T}$ spectra calculated in the blast-wave model are insensitive to the time parameters $\tau_{0}$ and $\Delta \tau$. The spectral shapes are furthermore insensitive to the spatial scale (i.e. $R_{y}$ ) of the source, though, as we see below, there is some small sensitivity to the spatial shape (i.e. $\frac{R_{y}}{R_{x}}$ ).

First, we study the importance of using quantum (as opposed to classical) statistics in the source function. Figure 3 shows $p_{T}$ spectra for pions and protons, treated as bosons and fermions, respectively. Model parameters were set to the "non-round" values listed in Table【 The sum in Equation 8 (and Equations 12 and 18) is over $n=1 \ldots N$; curves are shown for $N=1,2,3,4$. For parameter values in the range we study here, proton spectra are essentially independent of $N$. For $N>1$, the pion spectra are likewise robust against the value of $N$, though in the classical limit $(N=1)$, there is relatively lower yield at low $p_{T}$. (Note that all spectra are arbitrarily normalized to unity at $p_{T}=0$.) Calculations below use the truncation $N=2$.

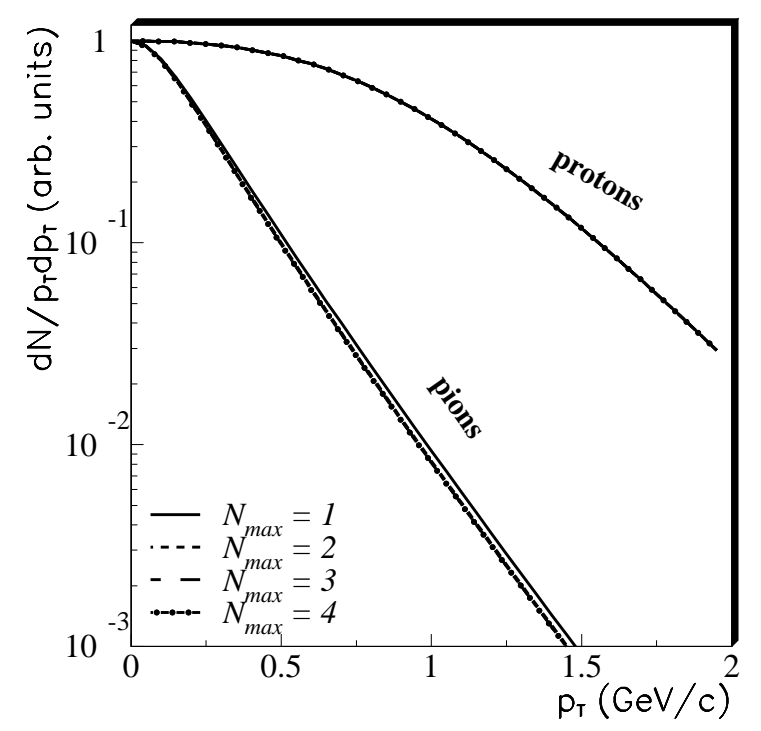

FIG. 3: Transverse momentum spectra for protons (upper curves) and pions (lower curves), as calculated by Equation [19] for several values of $N$, the maximum value of $n$ taken in the summation of Equation 8 see text for details. Parameters values correspond to the "non-round" source of Table 【 All spectra are arbitrarily normalized to unity at $p_{T}=0$.

\section{Spectra from Central Collisions}

Focusing first on central collisions (so that the flow anisotropy parameter $\rho_{2}=0$ and $R_{x}=R_{y}$ ), then, we need only consider the spectra sensitivity to the temperature and radial flow parameters $\rho_{0}$ and $T$, and to the surface diffusion $a_{s}$.

Fixing the transverse spatial density distribution to a box profile $\left(a_{s}=0\right)$, the evolution of spectral shapes for pions and protons are shown in Figures 4 and [5] as the temperature and radial flow parameter, respectively, are varied about nominal values of $T=100 \mathrm{MeV}$ and $\rho_{0}=0.9$. As has been noted previously [36], at low $p_{T}$, temperature variations affect the lighter pions more strongly, while variations in the collective flow boost produce a stronger effect on the heavier particles.

Next, we consider the effect of a finite surface diffuseness parameter $\left(a_{s} \neq 0\right)$, i.e., using the smoother spatial density distributions of Figure 1 Since we assume a transverse flow profile which increases linearly with radius (c.f. Equation 4), one trivial effect is that, for a fixed $\rho_{0}$, increasing $a_{s}$ will produce a larger average flow boost $\langle\rho\rangle$. The effect of increasing transverse flow was already explored directly in Figure [5 so we avoid this trivial effect here, and explore the effect of varying $a_{s}$, while keeping $\langle\rho\rangle$ constant [30].

The average transverse flow boost $\langle\rho\rangle=F\left(a_{s}\right) \cdot \rho_{0}$ 


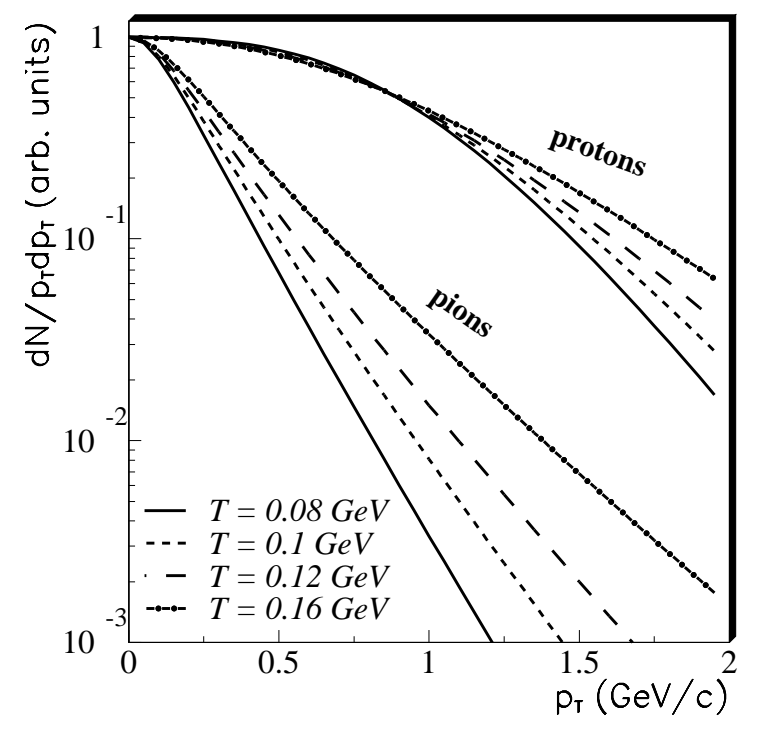

FIG. 4: Transverse momentum spectra for protons (upper curves) and pions (lower curves), as calculated by Equation 19 for several values of the temperature parameter $T$. Other parameters follow the "round" source defaults of Table I All spectra are arbitrarily normalized to unity at $p_{T}=0$.

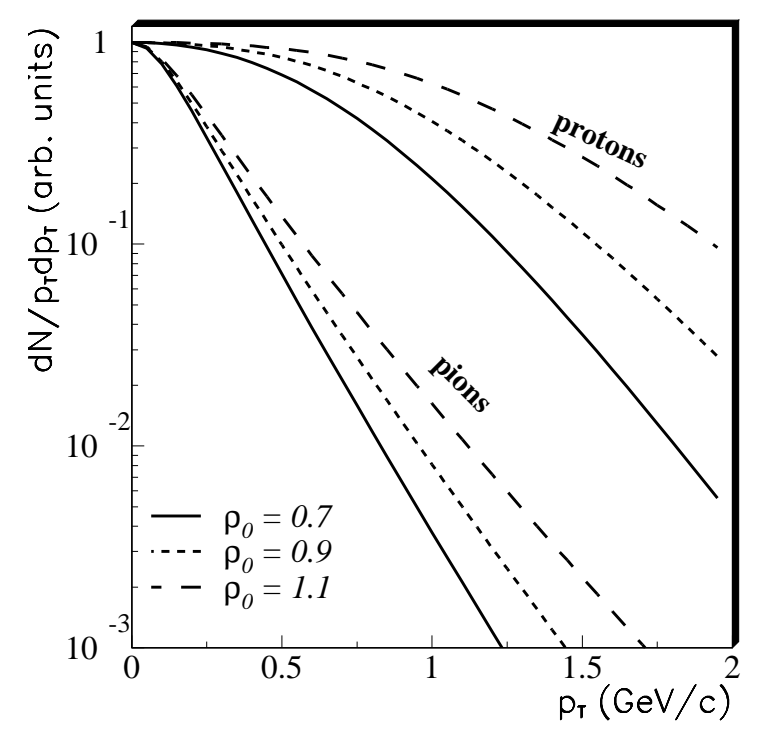

FIG. 5: Transverse momentum spectra for protons (upper curves) and pions (lower curves), as calculated by Equation [19] for several values of the radial flow parameter $\rho_{0}$. Other parameters follow the "round" source defaults of Table 【 All spectra are arbitrarily normalized to unity at $p_{T}=0$.

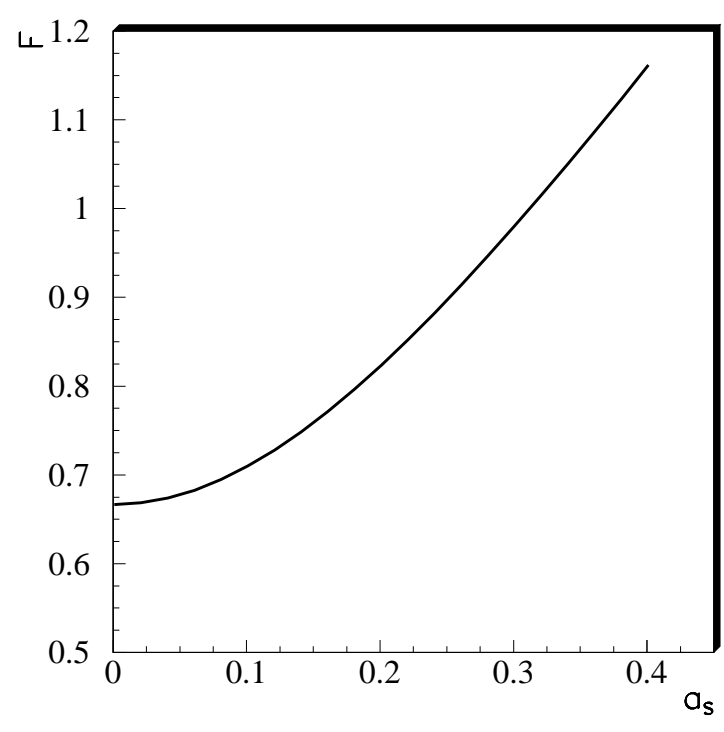

FIG. 6: The geometric constant of proportionality $F$ between the average transverse flow boost $\langle\rho\rangle$ and the blast-wave parameter $\rho_{0}$, as a function of the surface diffuseness $a_{s}$.

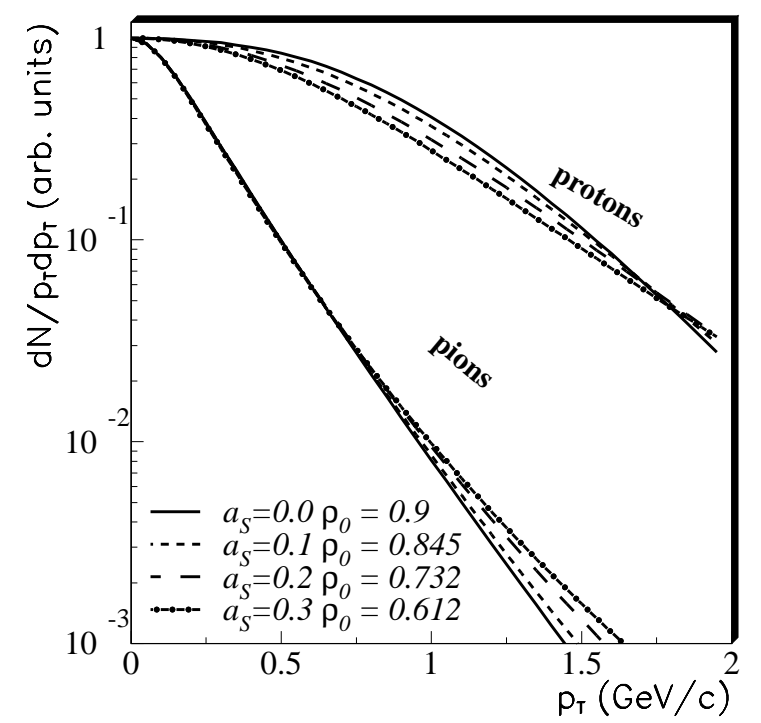

FIG. 7: Transverse momentum spectra for protons (upper curves) and pions (lower curves), as calculated by Equation 19] for several values of the surface diffuseness parameter $a_{s}$. The radial flow strength $\rho_{0}$ is co-varied; see text for details. Other parameters follow the "round" source defaults of Table【 All spectra are arbitrarily normalized to unity at $p_{T}=0$.

where the geometric proportionality constant

$$
F\left(a_{s}\right)=\frac{\int_{0}^{\infty} d x \frac{x^{2}}{1+\exp \left((x-1) / a_{s}\right)}}{\int_{0}^{\infty} d x \frac{x}{1+\exp \left((x-1) / a_{s}\right)}}
$$

is independent of $\rho_{0}$ or $R=R_{x}=R_{y}$. For the box profile, 
$F\left(a_{s}=0\right)=\frac{2}{3}$. Figure [6 shows this geometric factor as a function of the surface diffuseness.

Figure 7 shows the pion and proton spectra for various values of $a_{s}$. The radial flow strength $\rho_{0}$ was covaried with $a_{s}$ so that the average transverse flow boost was $\langle\rho\rangle=0.6$. To a first approximation, the spectral shapes depend only on the temperature $T$ and the average transverse flow boost $\langle\rho\rangle$. The residual dependence on the surface diffuseness parameter $a_{s}$ arises from the fact that while the average boost rapidity has been held constant, the spread of boost rapidities increases with increasing $a_{s}$ [30]. Thus, we observe qualitatively similar variations in the spectral shapes when $a_{s}$ increases (Figure 17), as when $T$ increases (Figure 4). The variations are not quantitatively identical since in the present case, the velocity spread is not thermal, and the particle velocity spread evolves differently with mass, depending on whether it arises from a boost spread or a thermal spread.

\section{Dependence of spectral shapes on source anisotropy}

Azimuthally-integrated $p_{T}$ spectra are often presented as a function of event centrality. For $b \neq 0$ collisions, the emitting source may have anisotropic structure $\left(R_{x} \neq R_{y}\right.$ and $\rho_{2} \neq 0$ in the present model). Thus, it is interesting to explore possible effects of these anisotropies.

For an azimuthally isotropic flow field $\left(\rho_{2}=0\right)$, the spectral shapes are insensitive to spatial anisotropies in the source (i.e. $R_{x} \neq R_{y}$ ). This is because the spectral shapes are determined by the distribution of boost velocities [30], which is unchanged by a shape change in our parameterization, if $\rho_{2}=0$.

For an azimuthally-symmetric spatial source $\left(R_{x}=\right.$ $R_{y}$ ), a very small variation in the $\phi_{p}$-integrated spectral shapes is observed when $\rho_{2}$ is changed from a value of 0.0 to 0.15 , as seen in Figure 8 This, again, is due to the slightly increased spread in boost velocities; source elements emitting in-plane boost a bit more, and out-ofplane a bit less. This effect becomes stronger in the presence of an out-of-plane spatial anisotropy $\left(R_{y} / R_{x}>1\right)$ as shown in Figure 9 In any case, the effects of "reasonable" source anisotropy on the shapes of azimuthallyintegrated spectra are very small.

Thus, we conclude that azimuthally-integrated $p_{T}$ spectra are largely insensitive to "reasonable" source anisotropies (see Section $\$ \nabla$ for "reasonable" ranges) and probe mainly the thermal motion $(T)$ and average transverse flow boost $(\langle\rho\rangle)$ of the source.

\section{B. Elliptic flow versus mass and $p_{T}$}

In the notation of Section ID the elliptic flow parameter $v_{2}$ is calculated as

$$
v_{2}\left(p_{T}, m\right)=\frac{\int_{0}^{2 \pi} d \phi_{p}\left\{\cos \left(2 \phi_{p}\right)\right\}_{0,0}(K)}{\int_{0}^{2 \pi} d \phi_{p}\{1\}_{0,0}(K)}
$$

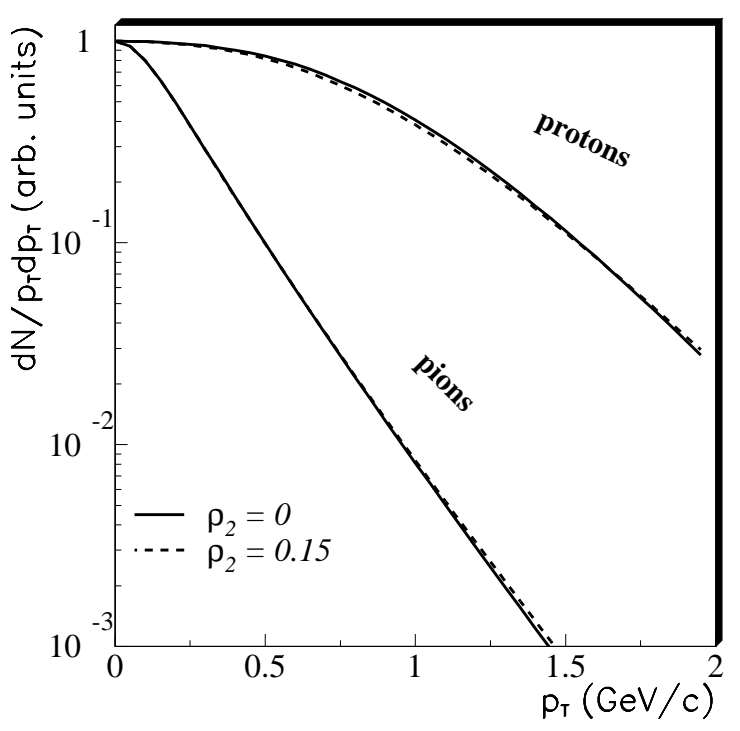

FIG. 8: Transverse momentum spectra for protons (upper curves) and pions (lower curves), as calculated by Equation 19 for an azimuthally symmetric flow field $\left(\rho_{2}=0\right)$ and an asymmetric field $\left(\rho_{2}=0.15\right)$. Other parameters follow the "round" source defaults of Table【 All spectra are arbitrarily normalized to unity at $p_{T}=0$.

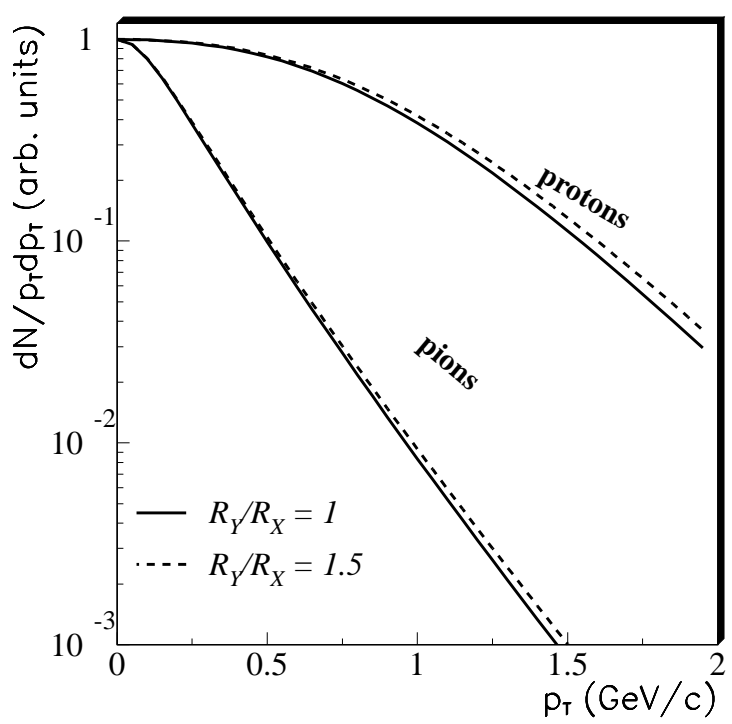

FIG. 9: Transverse momentum spectra for protons (upper curves) and pions (lower curves), as calculated by Equation 19 for an azimuthally anisotropic flow field $\left(\rho_{2}=0.15\right)$, and a spatially isotropic $\left(R_{y} / R_{x}=1\right)$ and anisotropic $\left(R_{y} / R_{x}=1.5\right)$ spatial distribution. Other parameters follow the "round" source defaults of Table凹

A finite $v_{2}$ arises from azimuthal anisotropies in the source $\left(R_{x} \neq R_{y}\right.$ and/or $\left.\rho_{2} \neq 0\right)$. As discussed below, however, the parameters $\rho_{0}$ and $T$ strongly affect its value 


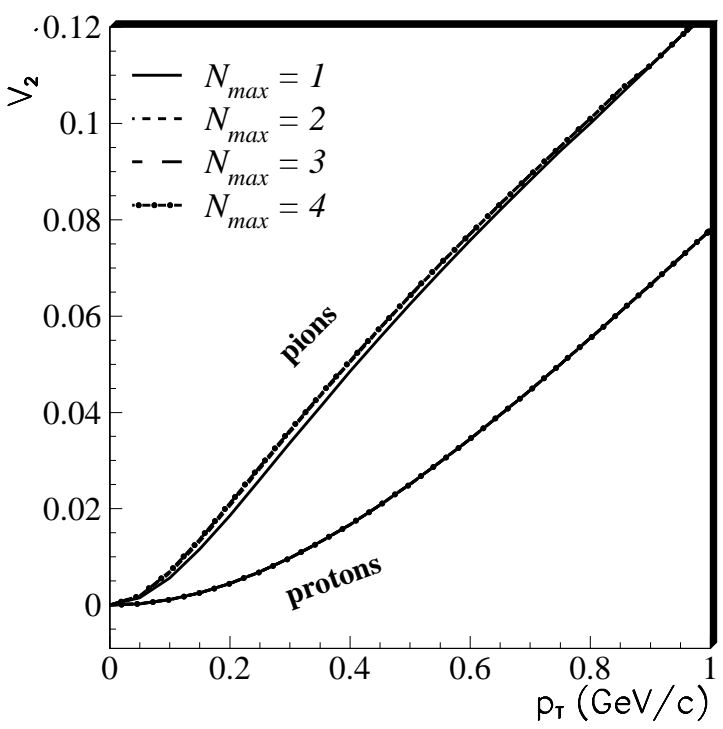

FIG. 10: Elliptic flow parameter $v_{2}$ for pions (upper curves) and protons (lower curves), as a function of transverse momentum, as calculated by Equation 21] for several values of $N$, the maximum value of $n$ taken in the summation of Equation 8 see text for details. Model parameters follow the "nonround" source defaults of Table $\square$

and evolution with $p_{T}$ and mass. In the present parameterization, $v_{2}$ is not sensitive to the overall spatial scale of the source $\left(R_{y}\right)$ or the time parameters $\tau_{0}$ and $\Delta \tau$.

The $v_{2}$ parameter depends non-trivially on both $p_{T}$ and particle mass [18, 38, 39, 40]. In this section, we explore the evolution of pion and proton $v_{2}$, as we vary the model parameters from nominal "non-round" values for non-central collisions (cf【).

As with the $p_{T}$ spectra of Section $11 \mathrm{~A}$ we first check the importance of quantum statistics. Figure [10 shows $v_{2}$ for pions and protons, for different values of $N$, where the sum in Equation 8 (and Equations 12 and 18) runs over $n=1 \ldots N$. Again, we find only a small difference for the pions between $N=1$ (classical limit) and $N=2$, beyond which $v_{2}$ is robust against further increases in $N$. Calculations here use $N=2$.

Figure [1] shows the evolution of $v_{2}$ as the temperature parameter $(T)$ is varied. For both particle types shown, the increased thermal smearing in momentum space, as $T$ is increased, leads to a reduced momentumspace anisotropy. The effect of the thermal smearing is greater for the lighter pions.

Less intuitive is the evolution of $v_{2}$ as the flow field $\left(\rho_{0}\right.$ or $\left.\rho_{2}\right)$ is varied. In Figures 12 and 13 the average transverse flow parameter $\rho_{0}$ is varied for an azimuthally isotropic $\left(R_{y} / R_{x}=1\right)$ and anisotropic $\left(R_{y} / R_{x}=13 / 11\right)$ shape, respectively. For the isotropic spatial distribution, we find that $v_{2}$ decreases as $\rho_{0}$ increases, for all $p_{T}$ and for both particle types. This is due to the decreasing relative amplitude of the oscillation in the flow field. Not

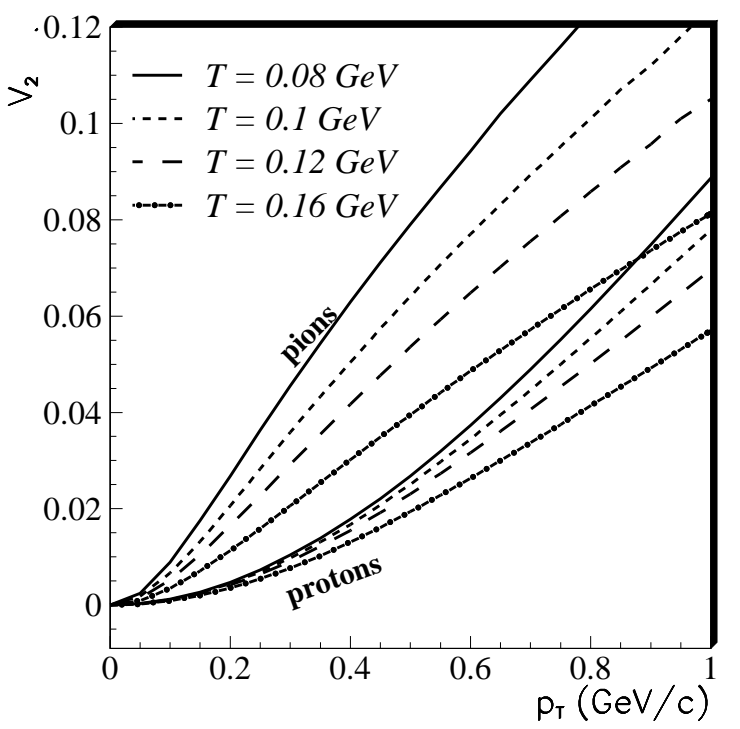

FIG. 11: Elliptic flow parameter $v_{2}$ for pions (upper curves) and protons (lower curves), as a function of transverse momentum, as calculated by Equation 21] for various values of the temperature parameter $T$. Other parameters follow the "non-round" source defaults of Table \

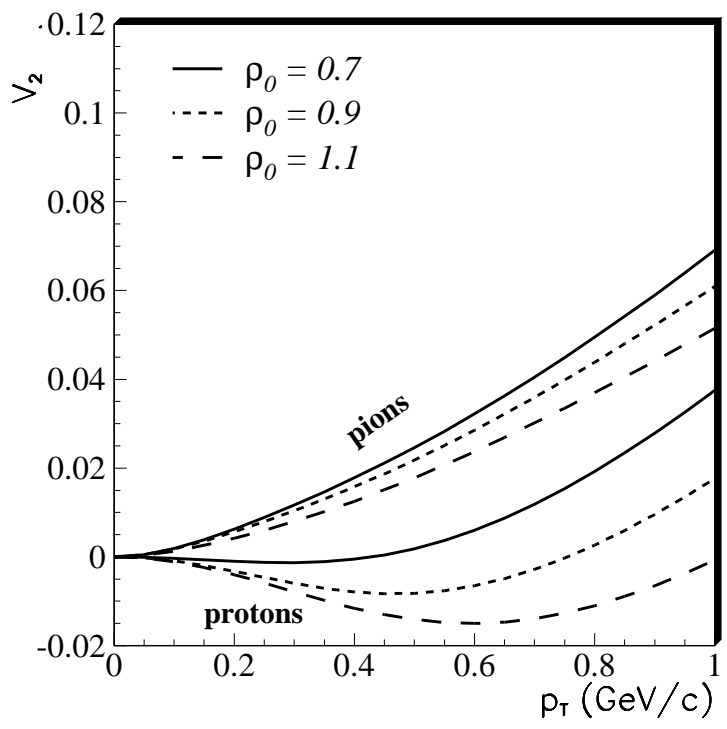

FIG. 12: Elliptic flow parameter $v_{2}$ for pions (upper curves) and protons (lower curves), as a function of transverse momentum, as calculated by Equation 21 for various values of the transverse flow parameter $\rho_{0}$. The source spatial distribution is assumed azimuthally-anisotropic $\left(R_{y}=R_{x}\right)$, but other parameters follow the "non-round" source defaults of Table【

surprisingly, the effect is larger for the heavier protons.

Indeed, it has been pointed out 18, 40, 41] that high radial flow (large $\rho_{0}$ ) can lead to negative values of $v_{2}$ 


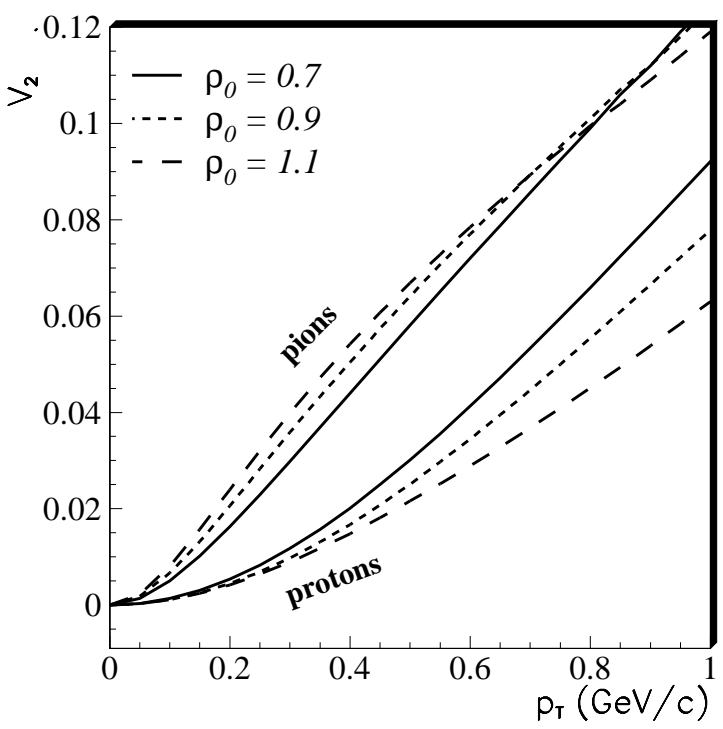

FIG. 13: Elliptic flow parameter $v_{2}$ for pions (upper curves) and protons (lower curves), as a function of transverse momentum, as calculated by Equation 21] for various values of the transverse flow parameter $\rho_{0}$. Other parameters follow the "non-round" source defaults of Table \

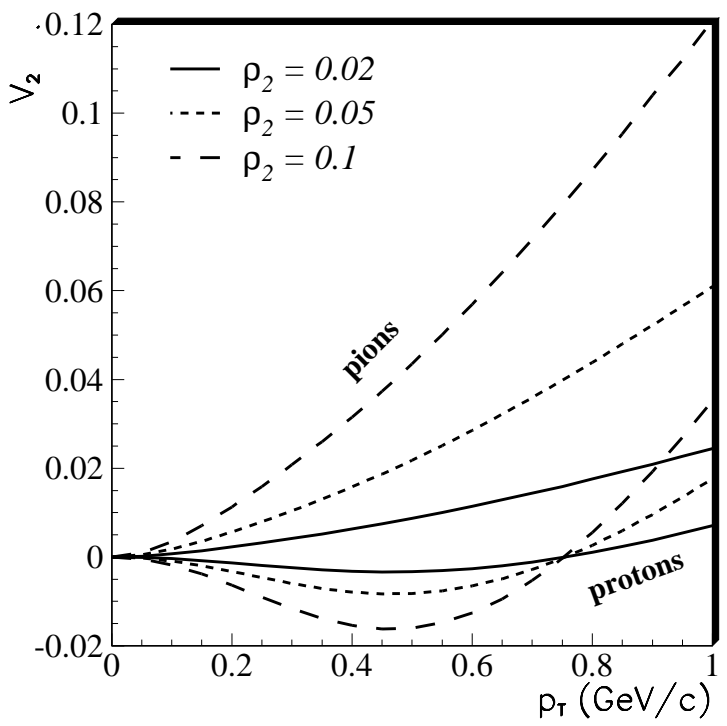

FIG. 14: Elliptic flow parameter $v_{2}$ for pions (upper curves) and protons (lower curves), as a function of transverse momentum, as calculated by Equation 21 for various values of the modulation in the transverse flow $\rho_{2}$. Other parameters follow the "round" (note: $R_{y}=R_{x}$ ) source defaults of Table

for heavy particles; this is clearly true for the protons in Figure 12 This negative $v_{2}$ reflects the depletion of the low- $p_{T}$ particle yield when most source elements are highly boosted transversely; this is also the origin of the

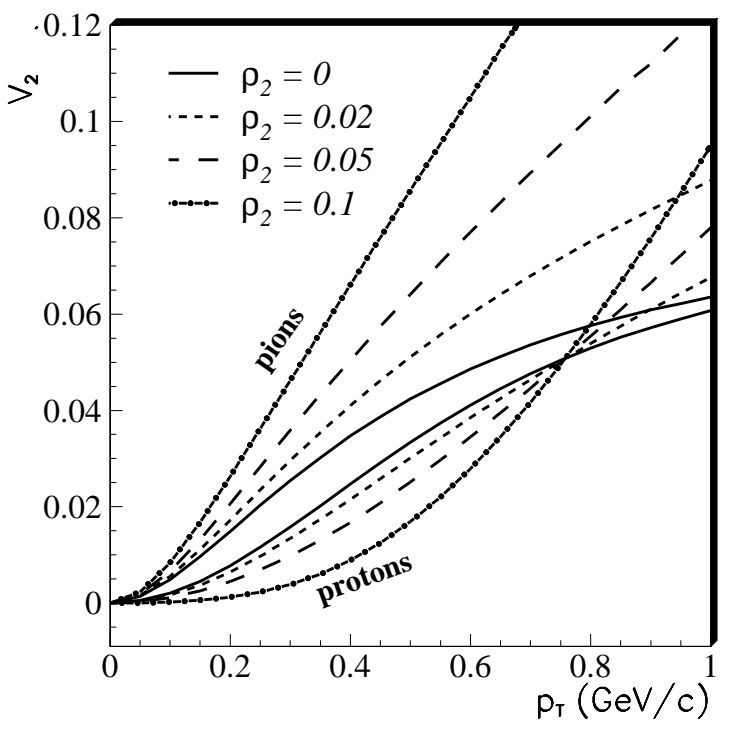

FIG. 15: Elliptic flow parameter $v_{2}$ for pions (upper curves) and protons (lower curves), as a function of transverse momentum, as calculated by Equation 21] for various values of the modulation in the transverse flow $\rho_{2}$. Other parameters follow the "non-round" source defaults of Table \

"shoulder arm" in the $p_{T}$ spectra (c.f. Section 【IIA). This depletion is larger in-plane (since the boost is higher, i.e. $\rho_{2}>0$ ), leading to negative $v_{2}$. At higher $p_{T}$, and/or lower flow strengths, the competing effect is dominant: the larger in-plane boost leads to more particles emitted in-plane, and $v_{2}>0$.

For a slightly anisotropic shape $\left(R_{y} / R_{x}=13 / 11\right.$; Figure 131), the $v_{2}$ parameter increases significantly for both protons and pions, due to the larger number of source elements boosted in-plane (c.f. Figure 2). The finite spatial asymmetry leads to an effect that tends to oppose the reduction of $v_{2}$ with increasing $\rho_{0}$, discussed above, and, for pions at low $p_{T}$, even reverses it in this case. Hence, at low $p_{T}$, increasing $\rho_{0}$ increases (decreases) $v_{2}$ for pions (protons).

We observe similar trends when $\rho_{0}$ is held fixed, and $\rho_{2}$ is varied. Figure [14 shows $v_{2}$ evolution for a spatially azimuthally-symmetric source $\left(R_{y} / R_{x}=1\right)$ and Figure 15 for a slightly asymmetric source $\left(R_{y} / R_{x}=13 / 11\right)$.

As mentioned above, a finite value of $v_{2}$ may be obtained for an azimuthally-symmetric flow field, if the spatial shape is asymmetric. Figure [16] shows the proton and pion $v_{2}$ for $\rho_{2}=0$ and $\rho_{0}=0.9$, for various values of $R_{y} / R_{x}$. The larger number of sources emitting inplane results in more particles measured in-plane (thus $\left.v_{2}>0\right)$. This momentum-space asymmetry saturates at $p_{T} \sim 1.0 \mathrm{GeV} / \mathrm{c}$ (for this set of parameters) and is relatively independent of particle mass. (We note that the $p_{T}$-scale in Figure [16] is larger than the other figures, in order to show the $v_{2}$ saturation effect.) This effect is similar to those previously discussed by Hou- 


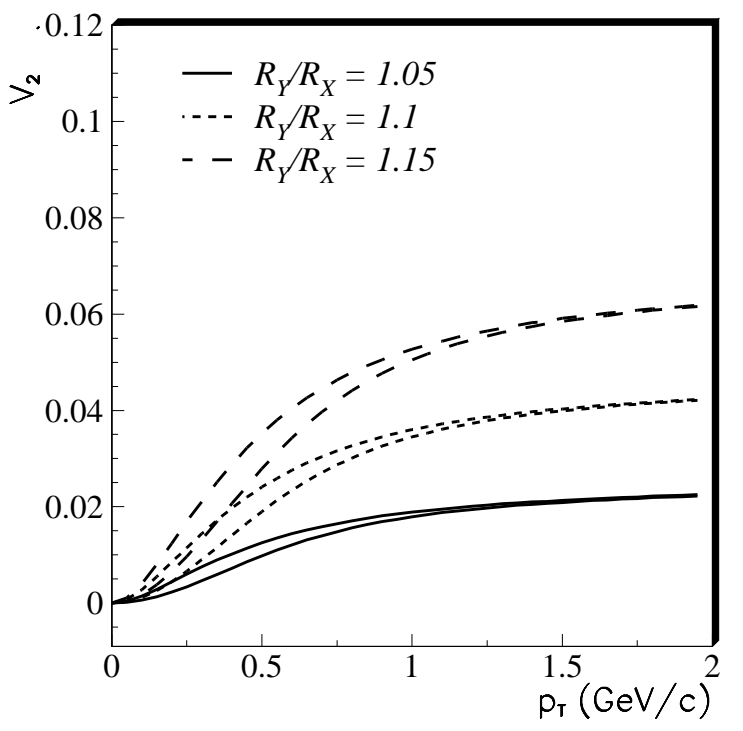

FIG. 16: Elliptic flow parameter $v_{2}$ for pions (upper curves) and protons (lower curves), as a function of transverse momentum, as calculated by Equation 21] for various values of the spatial anisotropy $\left(R_{y} / R_{x}\right)$. Elliptic flow parameter $v_{2}$ for pions (upper curves) and protons (lower curves), as a function of transverse momentum, as calculated by Equation 21 for various values of the modulation in the transverse flow $\rho_{2}$. Other parameters follow the "round" (note: $\rho_{2}=0$ ) source defaults of Table $\square$

vinen, et al, 87] and Shuryak [88]. However, we stress the non-zero $v_{2}$ parameter does not indicate "elliptic flow without transverse flow," as suggested in 87]. If transverse flow is turned off $\left(\rho_{0}=0\right)$, the spectra are thermal and isotropic, and $v_{2}=0$ for all $p_{T}$; space-momentum correlations (induced by $\rho \neq 0$ in our model) are required for a finite $v_{2}$. In the Houvinen model [87] it is the implementation of the Cooper-Frye freeze-out procedure (which creates an infinitely opaque source) which produces the space-momentum correlations, effectively generating "flow." Shuryak 88] implemented a parameter $\kappa$ which controlled the opaqueness; $v_{2}$ was found to increase with $\kappa$ until the source was essentially infinitely opaque.

Finally, we again explore the effect of "softening" the spatial source distribution $\Omega\left(r, \phi_{s}\right)$. Similar to the discussion surrounding Figure 7 it is clear that the most meaningful comparison comes from keeping the average value of the transverse boost (and its anisotropy) constant, not keeping the parameters $\rho_{0}$ and $\rho_{2}$ constant, as we vary $a_{s}$. Similar to the discussion of the $p_{T}$ spectra, we find in Figure 17 that $v_{2}$ is largely insensitive to the surface diffuseness parameter $a_{s}$.

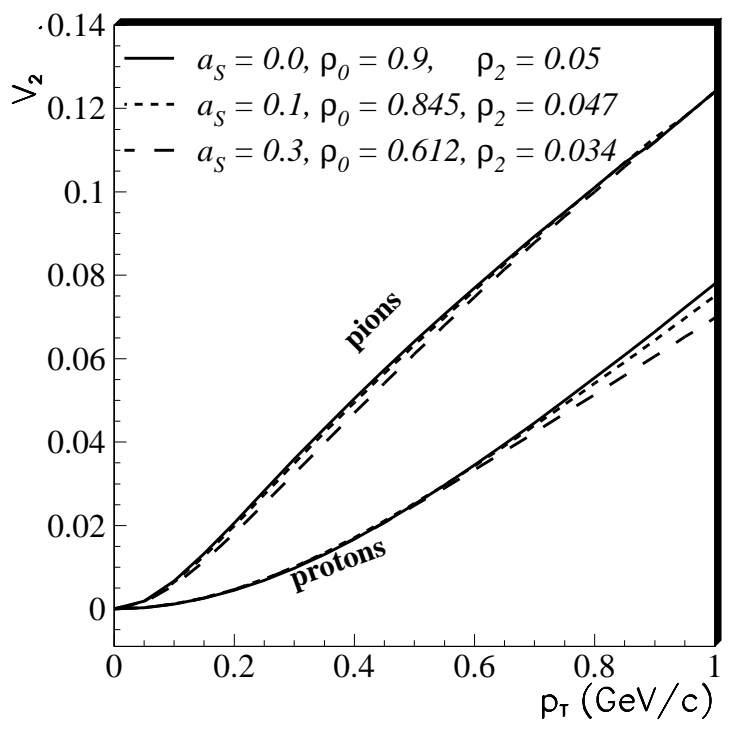

FIG. 17: Elliptic flow parameter $v_{2}$ for pions (upper curves) and protons (lower curves), as a function of transverse momentum, as calculated by Equation 21 for various values of the surface diffuseness parameter $a_{s}$. The values of $\rho_{0}$ and $\rho_{2}$ are co-varied with $a_{s}$ so that the average transverse flow and its average azimuthal modulation remain fixed; see text. Other parameters follow the "non-round" source defaults of Table I

\section{HBT radii versus $p_{T}$ and $\phi_{p}$}

The previous subsections have discussed momentumspace observables only, even though coordinate-space considerations came into play indirectly. The most direct experimental probes of the space-time structure of the freeze-out configuration are two-particle correlation functions in relative momentum 6, 69]. Here, we use the standard "out-side-long" coordinate system of Pratt and Bertsch 43], in which the long direction $\left(R_{l}\right)$ is parallel to the beam, the side direction $\left(R_{s}\right)$ is perpendicular to the beam and total pair momentum, and the out direction $\left(R_{o}\right)$ is perpendicular to the long and side directions.

For boost-invariant sources, the HBT radii $R_{o l}^{2}$ and $R_{s l}^{2}$ vanish by symmetry 48|. (For the more general case, see References [44, 47, 48, 49, 50].) Thus, we are left with four HBT radii, which are related to space-time variances 
as 6,44$]$

$$
\begin{aligned}
R_{s}^{2}= & \frac{1}{2}\left(\left\langle\tilde{x}^{2}\right\rangle+\left\langle\tilde{y}^{2}\right\rangle\right)-\frac{1}{2}\left(\left\langle\tilde{x}^{2}\right\rangle-\left\langle\tilde{y}^{2}\right\rangle\right) \cos \left(2 \phi_{p}\right) \\
& -\langle\tilde{x} \tilde{y}\rangle \sin \left(2 \phi_{p}\right) \\
R_{o}^{2}= & \frac{1}{2}\left(\left\langle\tilde{x}^{2}\right\rangle+\left\langle\tilde{y}^{2}\right\rangle\right)+\frac{1}{2}\left(\left\langle\tilde{x}^{2}\right\rangle-\left\langle\tilde{y}^{2}\right\rangle\right) \cos \left(2 \phi_{p}\right) \\
& +\langle\tilde{x} \tilde{y}\rangle \sin \left(2 \phi_{p}\right)-2 \beta_{\perp}\left(\langle\tilde{t} \tilde{x}\rangle \cos \phi_{p}+\langle\tilde{t} \tilde{y}\rangle \sin \phi_{p}\right) \\
& +\beta_{\perp}^{2}\left\langle\tilde{t}^{2}\right\rangle \\
R_{o s}^{2}= & \langle\tilde{x} \tilde{y}\rangle \cos \left(2 \phi_{p}\right)-\frac{1}{2}\left(\left\langle\tilde{x}^{2}\right\rangle-\left\langle\tilde{y}^{2}\right\rangle\right) \sin \left(2 \phi_{p}\right) \\
& +\beta_{\perp}\left(\langle\tilde{t} \tilde{x}\rangle \sin \phi_{p}-\langle\tilde{t} \tilde{y}\rangle \cos \phi_{p}\right), \\
R_{l}^{2}= & \left\langle\tilde{z}^{2}\right\rangle-2 \beta_{l}\langle\tilde{t} \tilde{z}\rangle+\beta_{l}^{2}\left\langle\tilde{t}^{2}\right\rangle,
\end{aligned}
$$

where

$$
\begin{array}{r}
\langle f(x)\rangle(K) \equiv \frac{\int d^{4} x f(x) S(x, K)}{\int d^{4} x S(x, K)} \\
\tilde{x}^{\mu} \equiv x^{\mu}-\left\langle x^{\mu}\right\rangle(K)
\end{array}
$$

We restrict our attention to correlations calculated in the Longitudinally Co-Moving System (LCMS), in which $Y=\beta_{l}=0$. In this case the last Equation 22 simplifies to

$$
R_{l}^{2}=\left\langle\tilde{z}^{2}\right\rangle
$$

In the notation of Section

$$
\overline{\{B\}_{j, k}} \equiv \frac{\{B\}_{j, k}}{\{1\}_{0,0}}
$$

the space-time correlations of interest are

$$
\begin{aligned}
\left\langle\tilde{x}^{2}\right\rangle & =\overline{\left\{x^{2}\right\}_{0,0}}-\overline{\{x\}_{0,0}}{ }^{2} \\
\left\langle\tilde{y}^{2}\right\rangle & =\overline{\left\{y^{2}\right\}_{0,0}}-\overline{\{y\}_{0,0}} \\
\langle\tilde{x} \tilde{y}\rangle & =\overline{\{x y\}_{0,0}}-\overline{\{x\}_{0,0}} \overline{\{y\}_{0,0}} \\
\langle\tilde{x} \tilde{t}\rangle & =\frac{\Delta \tau^{2}+\tau_{0}^{2}}{\tau_{0}} \cdot\left(\overline{\{x\}_{0,1}}-\overline{\{x\}_{0,0}} \overline{\{1\}_{0,1}}\right) \\
\langle\tilde{y} \tilde{t}\rangle & =\frac{\Delta \tau^{2}+\tau_{0}^{2}}{\tau_{0}} \cdot\left(\overline{\{y\}_{0,1}}-\overline{\{y\}_{0,0}} \overline{\{1\}_{0,1}}\right) \\
\left\langle\tilde{t}^{2}\right\rangle & =\left(3 \Delta \tau^{2}+\tau_{0}^{2}\right) \overline{\{1\}_{0,2}}-\left(\frac{\Delta \tau^{2}+\tau_{0}^{2}}{\tau_{0}}\right)^{2} \overline{\{1\}_{0,1}} \\
\left\langle\tilde{z}^{2}\right\rangle & =\left(3 \Delta \tau^{2}+\tau_{0}^{2}\right) \overline{\{1\}_{2,0}}
\end{aligned}
$$

We note that all quantities with space-time dimensions are explicitly shown in Equations [26] and in particular, all dependence on the timescale parameters $\tau_{0}$ and $\Delta \tau$.

The proper time of freeze-out is often estimated (e.g. 51]) by fitting the $m_{T}$-dependence of the measured $R_{l}$ radius, to a formula motivated by Sinyukov and collaborators 52, 53], and subsequently improved upon by others [54, 55]. They assumed boost-invariant longitudinal flow, but vanishing transverse flow $\left(\rho=0 \rightarrow \beta=\frac{m_{T}}{T}\right)$ and instantaneous freeze-out in proper time (i.e. $\Delta \tau=$ 0 ); they also simplified the formalism by using Boltzmann statistics (i.e. using only the first term in the summation in Equation 8). In this case, we find, in agreement with References 54, 55,

$$
R_{l}^{2}\left(m_{T}\right)=\tau_{0}^{2} \frac{T}{m_{T}} \times \frac{\mathrm{K}_{2}\left(\mathrm{~m}_{\mathrm{T}} / \mathrm{T}\right)}{\mathrm{K}_{1}\left(\mathrm{~m}_{\mathrm{T}} / \mathrm{T}\right)} .
$$

We note that the last term, which represents a correction to the original Sinyukov formula [52, 53], approaches unity for $\frac{m_{T}}{T} \rightarrow \infty$ but remains sizable $(\sim 1.5)$ for $\frac{m_{T}}{T} \sim 2$.

In general, the emission "homogeneity region" 59 (characterized by the correlation coefficients of Equation 26) and the HBT radii of Equation 22 depend on the pair momentum [6, 44]. For a boost-invariant system, this corresponds to dependences on $p_{T}$ and $\phi_{p}$. Figure 18 shows projections onto the transverse $(x-y)$ plane of the emission regions for pions with $p_{T}=0.3 \mathrm{GeV} / \mathrm{c}$ and $\phi_{p}=$ $0^{\circ}, 135^{\circ}$, for an anisotropic source with strong transverse flow. The flow generates strong space-momentum correlations, so that particles with higher $p_{T}$ tend to be emitted from the edge of the source, with $\phi_{p} \approx \phi_{s}$. Together with the finite extent of the overall source, this implies that, spatially, the emission region is often wider in the direction perpendicular to the particle motion (indicated by the arrows) than along the motion; this can have strong implications, e.g., for the difference between $R_{o}^{2}$ and $R_{s}^{2}$.

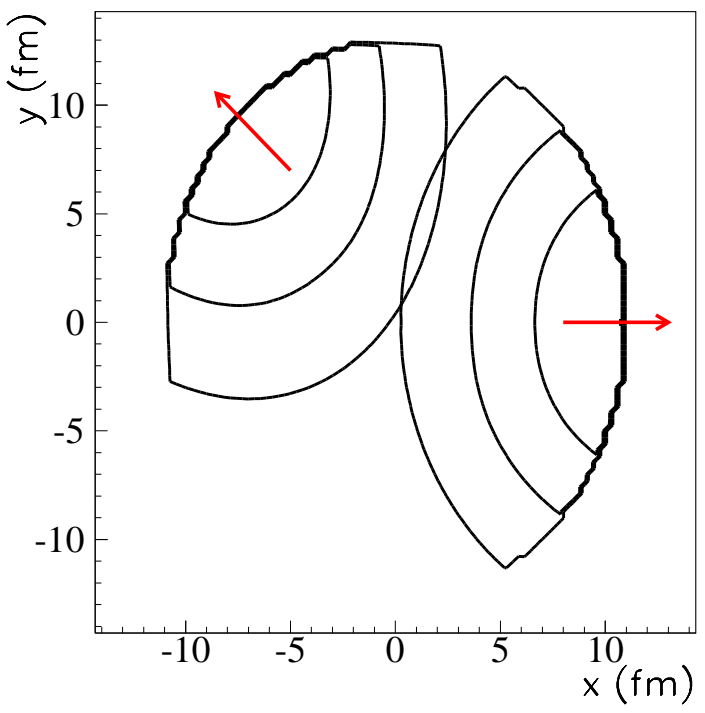

FIG. 18: (Color online) Emission probability contours, plotted on a linear scale, indicate emission zones for pions with $p_{T}=0.3 \mathrm{GeV} / \mathrm{c}$ at $\phi_{p}=0^{\circ}$ and $\phi_{p}=135^{\circ}$ (indicated by arrows), from a blast-wave source with "non-round" default parameters listed in Table

While the blast-wave parameterization provides direct access to the homogeneity region, the radii extracted from two-pion interferometry are compared to second order moments calculated as shown in equation [26] Such 

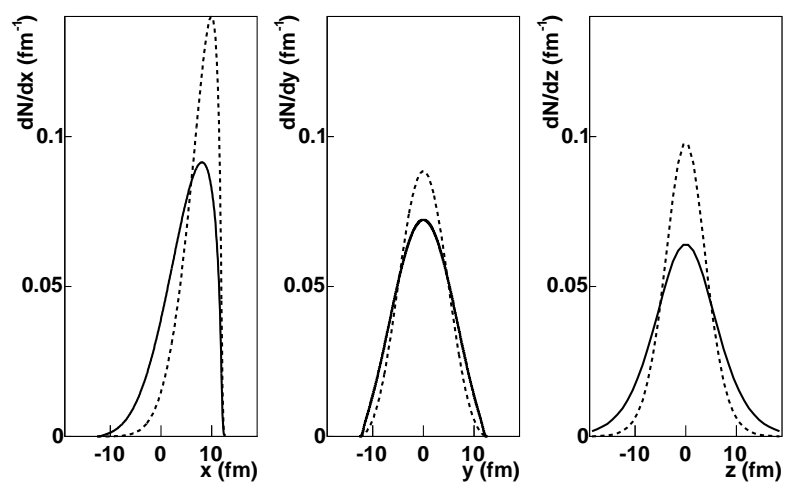

FIG. 19: Distribution of the pion spatial distribution in the direction $\mathrm{x}$ (left), $\mathrm{y}$ (middle) and $\mathrm{z}$ (right). Plain line: pion momentum $p_{x}=0.25 \mathrm{GeV} / \mathrm{c}$ and $p_{y}=0$. Dash line: pion momentum $p_{x}=0.5 \mathrm{GeV} / \mathrm{c}$ and $p_{y}=0$. Round source parameters of Table $\llbracket$ were used, with the exception of $a_{s}$ that was set to 0.01 .

comparison is strictly correct only if the homogeneity regions are Gaussian distributions. Figure [19 shows an example of spatial distributions along the 3 Cartesian directions for pions with two different momenta, $\vec{p}=(0.25$ $\mathrm{GeV} / \mathrm{c}, 0,0)$ and $\vec{p}=(0.5 \mathrm{GeV} / \mathrm{c}, 0,0)$; in this case, the $x-, y-$ and $z-$ directions correspond to "out," "side" and "long," respectively The $z$ distribution is nearly Gaussian; the difference between the $\sigma$ extracted from a Gaussian fit and the second order moment is on the order of a few percent. On the other hand, the $x$ and $y$ distributions are clearly not Gaussian. To estimate the level of distortion introduced by the non-Gaussian shape, we compare the second order moments with the Gaussian $\sigma$ extracted by fitting the peak of the spatial distributons. Indeed, it has been shown in [60] that calculating correlation function from models and fitting them as experimental data yields radii that are close to the Gaussian $\sigma$ extracted by fitting the peak of the spatial distributons. In both the $x$ - and $y$ - directions, we find that the Gaussian $\sigma$ are systematically larger than the second order moments by up to $20 \%$ (depending on the fit range) for pions at $p_{T}=0.25 \mathrm{GeV} / \mathrm{c}$. This discrepancy diminishes when increasing the transverse momentum or the particle mass. E.g. it is on the order of $10 \%$ for pions at $p_{T}=0.5 \mathrm{GeV} / \mathrm{c}$ or for kaons at $p_{T}=0.25 \mathrm{GeV} / \mathrm{c}$. Thus, comparing second order moments with the radii extracted from two-pion correlation functions may involve significant systematic errors at low transverse mass. This issue may be overcome by calculating correlation functions from the Blast Wave space-time distributions or by relying on imaging method to extract space-time distributions from the data [61]. Applying these methods is beyond the scope of this paper and we will thus carry on keeping in mind that systematic errors are associated with comparing HBT radii with second order moment at low transverse momentum.

On the other hand, resonance decay may introduce a core-halo pattern in the distribution of particle space- time emission points 62]. Indeed, some resonances decay sufficiently far away from the bulk of the system that their decay products emerge beyond the main homogeneity region. Such particles form a halo around the core of the source. However, to affect the extracted radii, the resonance lifetime needs to be short enough for the correlation to take place at a relative momentum accesible to the experiment. This lifetime is typically between 10 and $100 \mathrm{fm}$. The effect of resonance feed-down on radii measured by two-pion interferometry has been studied within a early version of the blast wave parameterization [63]. It was found that the $\omega$ meson leads to a halo effect, its $c \tau$ being $23.4 \mathrm{fm}$. The other resonances have lifetimes that are either too short (e.g. $\rho, \Delta)$ or too long (e.g. $\left.\eta, K_{s}^{0}\right)$. At RHIC energy, thermal models 64] show that about $10 \%$ of the pions come from $\omega$, which means that this effect should be rather small. The effect of the very long-lived $(c \tau>100 \mathrm{fm})$ resonances is usually assumed to reduce the so-called $\lambda$ parameter [65], which we do not discuss here.

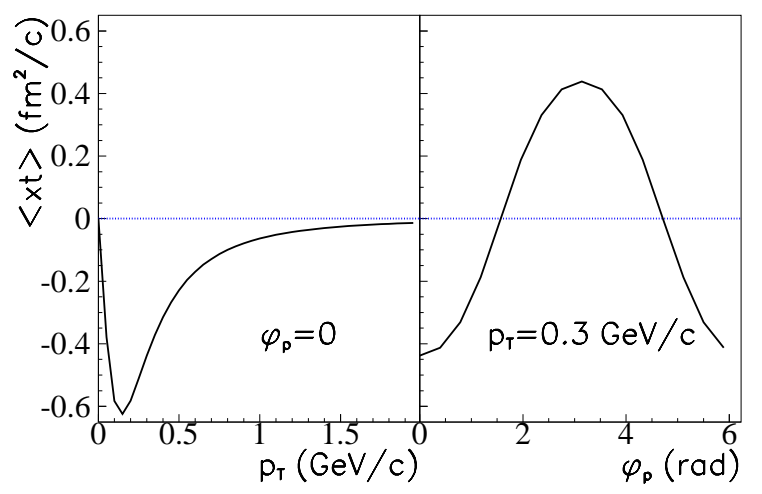

FIG. 20: (Color online) Space-time correlation $\langle\tilde{x} \tilde{t}\rangle$ as a function of $p_{T}$ for $\phi_{p}=0$ (left panel), and as a function of $\phi_{p}$ for $p_{T}=0.3 \mathrm{GeV} /$ c. "Non-round" source parameters of Table $\llbracket$ were used.

Also important are temporal effects, including spacetime correlations. The average $x-t$ correlation, quantified by $\langle\tilde{x} \tilde{t}\rangle$, for the same anisotropic source, is plotted in Figure 20] as a function of $p_{T}$ and $\phi_{p}$. In the left panel, $\phi_{p}=0^{\circ}$, and thus $x$ is the "out" direction. We first note that the correlation is negative, tending to increase $R_{o}^{2}$ for finite $\beta_{\perp}$ (see Equation 22); large and negative "out"- $t$ correlations at freezeout in some hydrodynamical models 66] have been a significant component of predicted large $R_{o}^{2} / R_{s}^{2}$ ratios. As required by symmetry [48], $\langle\tilde{x} \tilde{t}\rangle$ displays an odd-order cosine dependence on $\phi_{p}$; clearly the first-order component dominates here, as shown in the right panel of Figure 20. This $\cos \left(\phi_{p}\right)$-dependence is driven mostly by the $\cos \left(\phi_{s}\right)$ dependence of the $x$ coordinate, coupled with the radial flow which tends to make $\phi_{s} \approx \phi_{p}$ (c.f. Figure 18). However, we also note from the figure that the scale of these correlations is $\lesssim 1 \mathrm{fm}^{2} / \mathrm{c}$, which, as we shall see, is much smaller than the typical 
scale of $R_{o}^{2}$; hence, space-time correlations do not dominate in the blast-wave.

More important are the correlation coefficients $\langle\tilde{x} \tilde{y}\rangle$, $\left\langle\tilde{x}^{2}\right\rangle$ and $\left\langle\tilde{y}^{2}\right\rangle$, and their $p$-dependence. $\langle\tilde{x} \tilde{y}\rangle$ quantifies the transverse "tilt" of the emission zone with respect to the reaction plane. As required by symmetry [48], this tilt vanishes at $\phi_{p}=0$, while, in the present case with $R_{y}>R_{x}$, it is positive for $\phi_{p}=135^{\circ}$ (see Figure 18). That $\left\langle\tilde{x}^{2}\right\rangle$ and $\left\langle\tilde{y}^{2}\right\rangle$ also depend on $\phi_{p}$ is likewise clear from Figure 18 As we shall see, the $\phi_{p}$-dependence of $\langle\tilde{x} \tilde{y}\rangle,\left\langle\tilde{x}^{2}\right\rangle$, and $\left\langle\tilde{y}^{2}\right\rangle$, combined with the explicit $\phi_{p^{-}}$ dependences in Equation 22. drive the oscillations in HBT radii which we will study.

At this point, it is worthwhile to mention that the emission zone ("homogeneity region") and the correlation coefficients $\left(\left\langle\tilde{x}^{2}\right\rangle,\left\langle\tilde{y}^{2}\right\rangle,\langle\tilde{x} \tilde{y}\rangle,\langle\tilde{x} \tilde{t}\rangle,\langle\tilde{y} \tilde{t}\rangle\right)$ will vary with $\phi_{p}$ also for an azimuthally isotropic source $\left(R_{x}=R_{y}, \rho_{2}=\right.$ $0)$. However, of course, the measured HBT radii will be $\phi_{p}$-independent. This arises due to cancellation and combination of terms in Equations 22 For $R_{s}^{2}$, the first term becomes $\phi_{p}$-independent, and the second and third terms combined are $\phi_{p}$-independent $\left(\left(\left\langle\tilde{x}^{2}\right\rangle-\left\langle\tilde{y}^{2}\right\rangle\right) \propto \cos \left(2 \phi_{p}\right)\right.$ and $\left.\langle\tilde{x} \tilde{y}\rangle \propto \sin \left(2 \phi_{p}\right)\right)$. For $R_{o}^{2}$, the story is the same for the first three terms, while the fourth term becomes $\phi_{p^{-}}$ independent as $\langle\tilde{x} \tilde{t}\rangle \propto \cos \left(\phi_{p}\right)$ and $\langle\tilde{y} \tilde{t}\rangle \propto \sin \left(\phi_{p}\right)$. Meanwhile, for $R_{o s}^{2}$, the first and second terms cancel each other, as do the two components of the third term; hence $R_{o s}^{2}=0$ for azimuthally-symmetric sources. We mention these points here because several of the exact cancellations and combinations which hold for an isotropic source, continue to hold approximately even when the geometry or flow field is anisotropic.

We turn now to a detailed study of the observable HBT radius parameters, and the effects of varying blast-wave parameters. For a boost-invariant system, the symmetryallowed $\phi_{p}$ oscillations of the (squared) HBT radii are 48

$$
\begin{aligned}
R_{s}^{2}\left(p_{T}, \phi_{p}\right) & =R_{s, 0}^{2}\left(p_{T}\right)+2 \sum_{n=2,4,6, \ldots} R_{s, n}^{2}\left(p_{T}\right) \cos \left(n \phi_{p}\right), \\
R_{o}^{2}\left(p_{T}, \phi_{p}\right) & =R_{o, 0}^{2}\left(p_{T}\right)+2 \sum_{n=2,4,6, \ldots} R_{o, n}^{2}\left(p_{T}\right) \cos \left(n \phi_{p}\right), \\
R_{o s}^{2}\left(p_{T}, \phi_{p}\right) & =2 \sum_{n=2,4,6, \ldots} R_{o s, n}^{2}\left(p_{T}\right) \sin \left(n \phi_{p}\right), \\
R_{l}^{2}\left(p_{T}, \phi_{p}\right) & =R_{l, 0}^{2}\left(p_{T}\right)+2 \sum_{n=2,4,6, \ldots} R_{l, n}^{2}\left(p_{T}\right) \cos \left(n \phi_{p}\right),
\end{aligned}
$$

where the HBT radii Fourier coefficients $R_{\mu, n}^{2}(\mu=$ $o, s, o s, l$ and $n=0,2,4, \ldots)$ are $\phi_{p}$-independent.

Figure 21] shows the $p_{T}$ and $\phi_{p}$ dependence of HBT radii calculated for a blast-wave source with a slightly anisotropic flow field and shape. In addition to an overall decrease in the average value of the HBT radii with increasing $p_{T}$, we observe significant oscillations in the transverse radii $R_{o}^{2}, R_{s}^{2}, R_{o s}^{2}$, and smaller oscillations in $R_{l}^{2}$.

The Fourier coefficients may be calculated as

$$
R_{\mu, n}^{2}\left(p_{T}\right)=\left\{\begin{array}{ll}
\left\langle R_{\mu}^{2}\left(p_{T}, \phi_{p}\right) \cos \left(n \phi_{p}\right)\right\rangle & (\mu=o, s, l) \\
\left\langle R_{\mu}^{2}\left(p_{T}, \phi_{p}\right) \sin \left(n \phi_{p}\right)\right\rangle & (\mu=o s)
\end{array} .\right.
$$

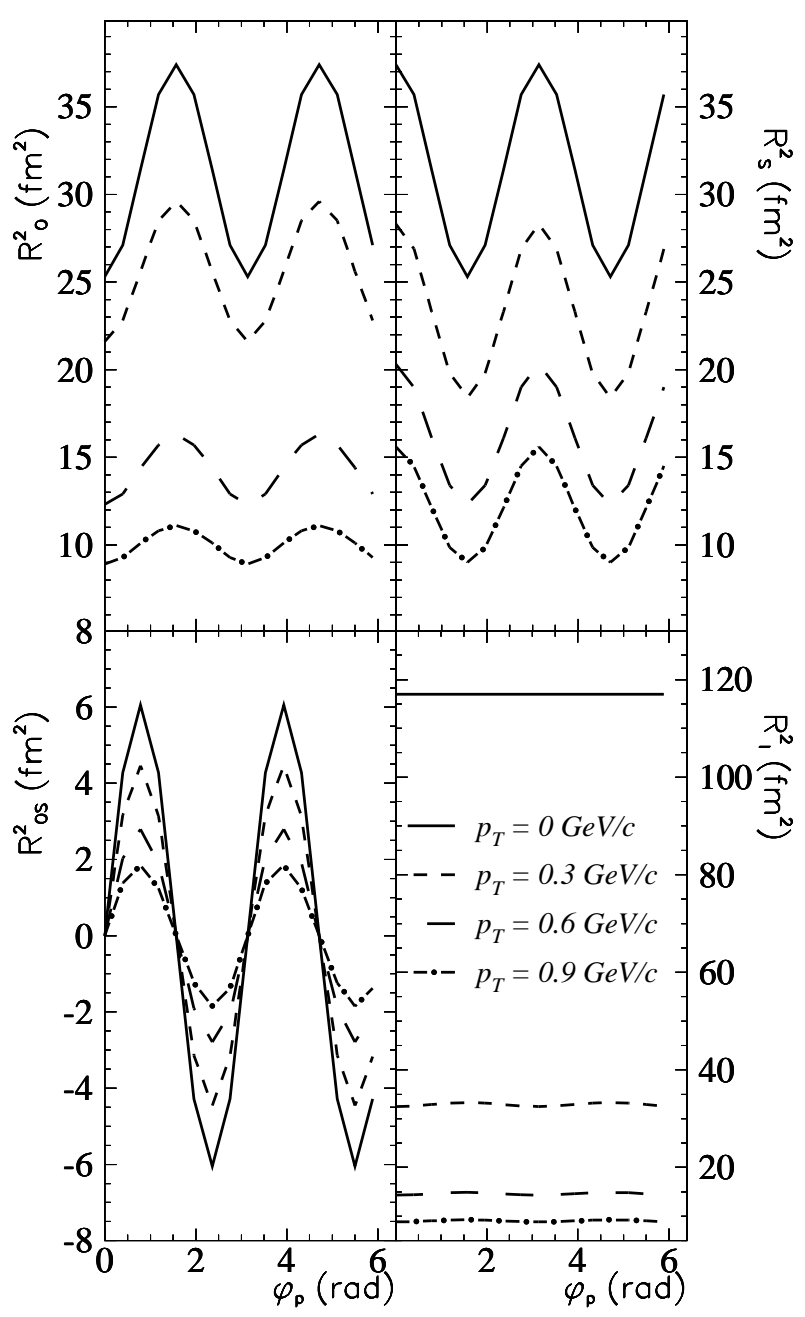

FIG. 21: Squared HBT radius parameters calculated with Equations 26 and 22 from a blast-wave source with the "nonround" default parameters of Table \ Squared radii for various cuts in $p_{T}$ are plotted versus $\phi_{p}$, the emission angle which respect to the event plane.

In the present model, we find that oscillation amplitudes above $2^{\text {nd }}$ order are very small in all cases considered $\left(\left|R_{\mu, 4}^{2} / R_{\mu, 2}^{2}\right| \lesssim 0.01\right)$. Therefore, the $\phi_{p}$-dependence of the HBT radii at a given $p_{T}$ is essentially encapsulated in seven numbers: the $0^{\text {th }}$ - and $2^{\text {nd }}$-order Fourier coefficients of $R_{o}^{2}, R_{s}^{2}$, and $R_{l}^{2}$, and the $2^{\text {nd }}$-order Fourier coefficient of $R_{o s}^{2}$. We henceforth explore the evolution of the $p_{T^{-}}$ dependence of these seven numbers, as model parameters are varied.

Figure 22 shows the Fourier coefficients for $n=0,2$, corresponding to a blast-wave source with $T=0.1 \mathrm{GeV}$, an isotropic flow field $\left(\rho_{0}=0.9, \rho_{2}=0\right)$, a box profile $\left(a_{s}=0\right)$, and time parameters $\tau_{0}=9 \mathrm{fm} / \mathrm{c}, \Delta \tau=2 \mathrm{fm} / \mathrm{c}$. Here, the average transverse size of the source $\left(R_{x}^{2}+R_{y}^{2}\right)$ was held fixed, while the shape $\left(R_{y} / R_{x}\right)$ was varied.

The $0^{\text {th }}$-order Fourier coefficients (corresponding to the 


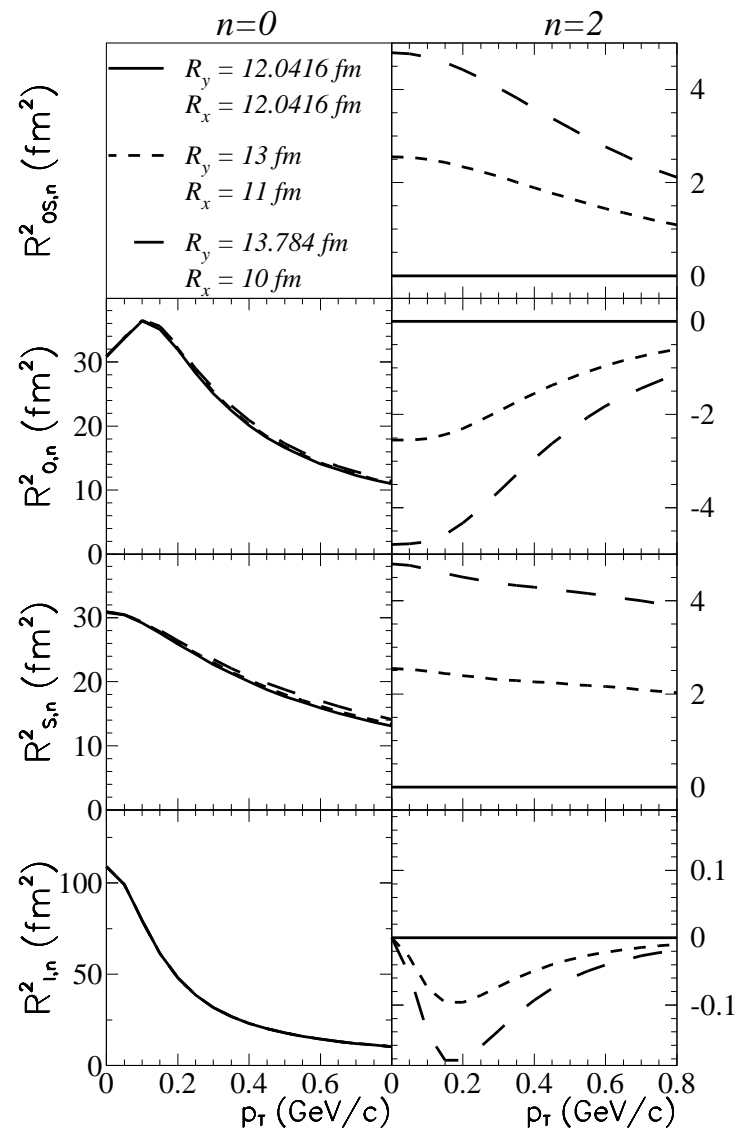

FIG. 22: Fourier coefficients of the $\phi_{p}$-dependence of the squared HBT radii, as calculated by Equation 29$] 0^{\text {th }}$ and $2^{\text {nd }}$-order Fourier coefficients are plotted in the left and right panels, respectively. The transverse shape of the source (i.e. $R_{y} / R_{x}$ ) was varied, while $R_{y}^{2}+R_{x}^{2}$ was held fixed. "Round" source values from Table $\llbracket$ are used for the other parameters.

radii $R_{o}, R_{s}$, and $R_{l}$ usually measured by experimentalists) are sensitive only to the average scale, not the shape, of the source. The average values of the transverse radii $R_{\rho 0}^{2}$ and $R_{s, 0}^{2}$ fall with increasing $p_{T}$ due to radial flow 6, 55] (c.f. Figure 31). At intermediate values of $p_{T}, R_{o, 0}^{2}>R_{s, 0}^{2}$ due to finite timescale effects (cf Figures 29] and [30), but at high $p_{T}, R_{o, 0}^{2}<R_{s, 0}^{2}$ (i.e. $R_{o} / R_{s}<1$ ), in qualitative agreement with experimental data 51, 67. The boost-invariant longitudinal flow produces the strong decrease of $R_{l, 0}^{2}$ with $p_{T}[\underline{6}, 52,53,54,55$.

Richer detail is seen in the oscillations of the HBT radii, quantified by Fourier coefficients $R_{\mu, 2}^{2}$ in the righthand panels of Figure 22 Here, the elliptical shape of the source is explicitly clear. The signs of the $2^{\text {nd }}$ order Fourier coefficients of the transverse radii directly reflect the out-of-plane-extended source geometry when $R_{y}>R_{x} . R_{o s, 2}^{2}$ has a similar geometric interpretation, in terms of the $\phi_{p}$-evolution of the "tilt" of the homo-

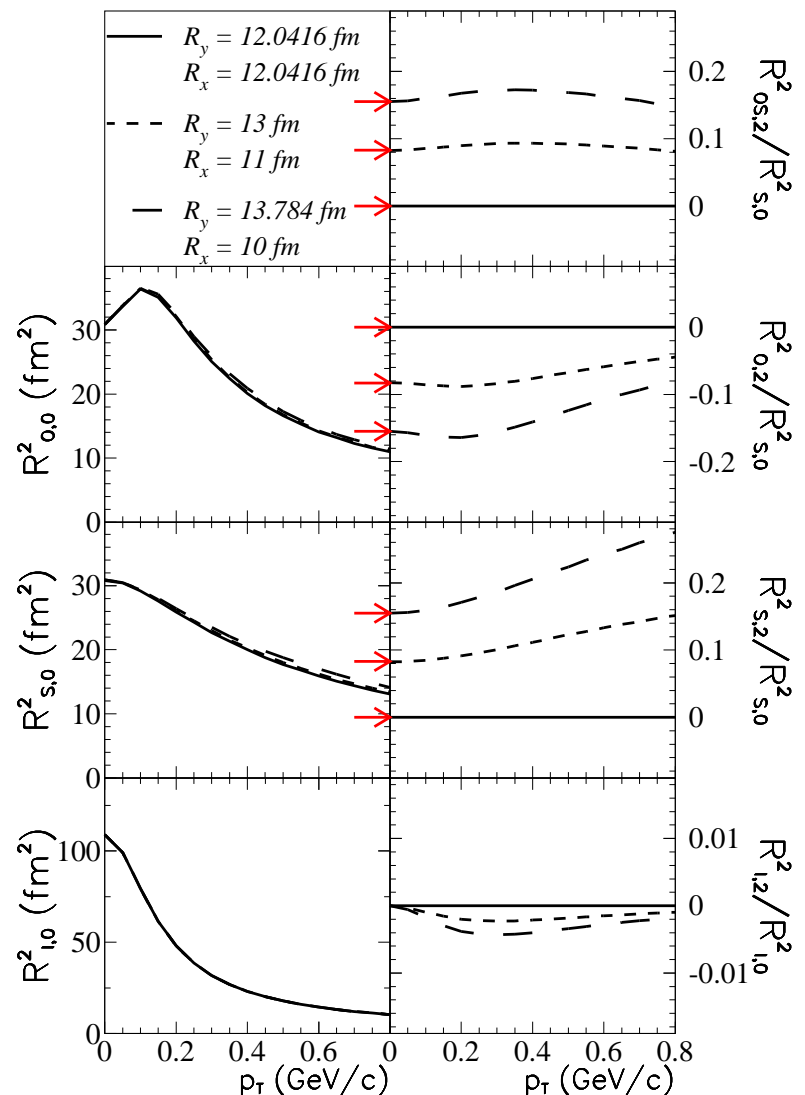

FIG. 23: (Color online) Identical data as in Figure 22 except the right panels show ratios of $2^{\text {nd }}$-order and $0^{\text {th }}$ order Fourier coefficients of the $\phi_{p}$-dependence of the squared HBT radii. Arrows indicate values calculated from Equation 32 see text for details.

geneity region [23]. The relatively small oscillations in $R_{l}^{2}$ arise not directly from geometry, but instead from transverse flow gradients, which slightly reduce $R_{l}^{2}$ [55]. In the present example, the transverse flow increases linearly from 0 (at the center) to $\rho_{0}=0.9$ (at the edge of the source), independent of boost angle $\phi_{b}$. However, when $R_{y}>R_{x}$, the flow gradient is larger for source elements boosted in-plane, leading to slightly greater reduction of $R_{l}^{2}$ when $\phi_{p}=0$; hence $R_{l, 2}^{2}<0$.

Finally, we recall that the flow field is isotropic $\left(\rho_{2}=0\right)$ and so all $\phi_{p}$-dependence arises from geometry here. Thus, if the values of $R_{y}$ and $R_{x}$ are interchanged (corresponding to in-plane-extended sources), $R_{\mu, 0}^{2}$ would remain unchanged, and $R_{\mu, 2}^{2}$ would simply change in sign in Figure 22

As discussed above, transverse flow-induced spacemomentum correlations tend to decrease homogeneity lengths as $p_{T}$ increases. When combined with other effects (e.g. temporal effects), non-trivial $p_{T}$ dependences of the HBT radii result. The $p_{T}$-dependences of the $\phi_{p}$-averaged values $R_{\mu, 0}^{2}$ have been discussed exten- 
sively [6, [5]. Meanwhile, the $p_{T}$-dependences of the oscillation amplitudes $\left(R_{\mu, 2}^{2}\right)$ shown in the right panels of Figure 22 have not been explored previously and may be non-trivial in principle.

It was suggested [68] that the $p_{T}$-dependence of $\left|R_{\mu, 2}^{2}\right|$ might be driven largely by the same effects which generate the $p_{T}$-dependence of $R_{\mu, 0}^{2}$, and hence the most efficient and direct way to study the source is to plot $R_{\mu, 0}^{2}$, which encode scale information, and then the $r a$ tio of $2^{\text {nd }}$ - to $0^{\text {th }}$-order Fourier coefficients, which would encode geometric and dynamic anisotropy. This is an excellent suggestion, though consideration must be given to the appropriate scaling. First, we consider the transverse radii $R_{o}^{2}, R_{s}^{2}$ and $R_{o s}^{2}$. The radii $R_{o}^{2}$ and $R_{o s}^{2}$ encode both transverse geometry and temporal information. As discussed above, space-time (e.g. $x-t$ ) correlations are small in magnitude, and furthermore affect the HBT radii in combinations which tend to cancel any $\phi_{p}$-dependence. Therefore, we expect $R_{o, 0}^{2}, R_{s, 0}^{2}, R_{s, 2}^{2}, R_{o, 2}^{2}$ and $R_{o s, 2}^{2}$ to contain geometric contributions, while temporal contributions are significant only for $R_{o, 0}^{2}$. In this case, the appropriate ratios to study are $R_{o, 2}^{2} / R_{s, 0}^{2}, R_{s, 2}^{2} / R_{s, 0}^{2}$ and $R_{o s, 2}^{2} / R_{s, 0}^{2}$. Indeed, we find numerically that these are the ratios least affected by the overall scale of the homogeneity region, which varies both with $p_{T}$ and with model parameter. The oscillation strength $R_{l, 2}^{2}$ of the longitudinal radius, on the other hand, is entirely due to implicit $\phi_{p}$ dependences driven by space-momentum correlations; these same correlations affect $R_{l, 0}^{2}$. Hence, the appropriate ratio to study in this case is $R_{l, 2}^{2} / R_{l, 0}^{2}$.

In Figure 23 we show these ratios for the same sources as were plotted in Figure 22 The $p_{T}$-dependence of the ratios is significantly less than that of the oscillation strengths $R_{\mu, 2}^{2}$, as anticipated, due to the fact that the latter is driven largely by space-momentum correlations reducing the spatial scale of the homogeneity region.

Going further, we may recall that in the special case of vanishing space-momentum correlations $(\rho=0$ or $T=\infty)$, the transvserse radii oscillate with identical strengths $\left(R_{o s, 2}^{2}=R_{s, 2}^{2}=-R_{o, 2}^{2}\right)$, and the in-plane and out-of-plane extents of the source may be directly extracted [44, 47, 48, 49], as the "whole source" is viewed from every angle. In that special case, independent of $p_{T}$

$$
\begin{aligned}
R_{s, 0}^{2}=\frac{1}{2}\left(\left\langle\tilde{y}^{2}\right\rangle+\left\langle\tilde{x}^{2}\right\rangle\right) & =\frac{1}{8} \cdot\left(R_{y}^{2}+R_{x}^{2}\right) \\
R_{o s, 2}^{2}=R_{s, 2}^{2}=-R_{o, 2}^{2} & =\frac{1}{4}\left(\left\langle\tilde{y}^{2}\right\rangle-\left\langle\tilde{x}^{2}\right\rangle\right) \\
& =\frac{1}{16}\left(R_{y}^{2}-R_{x}^{2}\right)
\end{aligned}
$$

so that

$$
\frac{R_{o s, 2}^{2}}{R_{s, 0}^{2}}=\frac{R_{s, 2}^{2}}{R_{s, 0}^{2}}=-\frac{R_{o, 2}^{2}}{R_{s, 0}^{2}}=\frac{1}{2} \cdot \frac{R_{y}^{2}-R_{x}^{2}}{R_{y}^{2}+R_{x}^{2}} \equiv \frac{\epsilon}{2}
$$

In the presence of flow, however, HBT radii measured at momentum $\vec{p}$ reflect homogeneity lengths which in principle may vary nontrivially both with $p_{T}$ and $\phi_{p}$. While we find that non-vanishing flow violates the $p_{T^{-}}$ independence of $R_{s, 0}^{2}, R_{o, 2}^{2}, R_{s, 2}^{2}$ and $R_{o s, 2}^{2}$ (and thus Equations [30 and 31), Equation 32] remains remarkably robust. As seen in the next several Figures, the ratios $R_{o, 2}^{2} / R_{s, 0}^{2}, R_{s, 2}^{2} / R_{s, 0}^{2}$ and $R_{o s, 2}^{2} / R_{s, 0}^{2}$, largely independent of $p_{T}$, provide an estimate of the source ellipticity $\epsilon$. Arrows to the left of the panels for $R_{o, 2}^{2} / R_{s, 0}^{2}, R_{s, 2}^{2} / R_{s, 0}^{2}$ and $R_{o s, 2}^{2} / R_{s, 0}^{2}$ in Figures 233 34 indicate $\epsilon / 2$ for the sources used.

Now that we have established the quantities to be examined in this Section, we briefly check the importance of using quantum, rather than classical, statistics in the source function of Equation 8 Setting the parameter values to correspond to the "non-round" source of Table \ we plot in Figure 24 the Fourier coefficients corresponding to different values of $N$, where the summation in Equation 8 (and Equations [2] and 18) is over $n=1 \ldots N$. Once again, we find a small difference between the curves for $N=1$ and $N>1$, while inclusion of higher terms in the summation have essentially no effect. Blast-wave calculations in this Section correspond to $N=2$.

Recently, Heinz and Kolb, in a hydrodynamic model, calculated HBT radii as a function of $\phi_{p}$ for non-central collisions 23]. They used two different Equations of State and initial conditions: one ("RHIC") is appropriate for soft physics at RHIC energies, and has successfully reproduced momentum-space observables [9]; the other ("IPES") assumes extremely high initial energy densities, perhaps appropriate for collisions at LHC energies.

It is worthwhile to point out that even the "RHIC" hydrodynamic calculations fail to reproduce azimuthallyintegrated HBT data 9]; here, however, we simply investigate the connection between the freeze-out geometry and oscillations of the HBT radii. Both calculations result in a freeze-out configuration, integrated over $p_{T}$, which is rather sharp-edged in transverse coordinatespace; thus, we may extract surface radii $R_{x}$ and $R_{y}$ to calculate $\epsilon$.

Figure 25] shows the same quantities as plotted in Figure 23. but extracted from these hydrodynamic calculations. The "RHIC" source, which is geometrically extended out-of-plane $\left(R_{y}>R_{x}\right.$, resulting in a positive $\epsilon)$ generates oscillations in the transverse radii with the same phase as out-of-plane sources in blast-wave calculations. For this source, the connection between $\epsilon$ and the radius oscillations (Equation 32) is most robust for $R_{s, 2}^{2}$ and least well-satisfied for $R_{o, 2}^{2}$, an effect not observed in the blast-wave. However, our blast-wave parameterization does not include explicit $\phi_{p}$ dependence of the temporal scale, which would affect $R_{o, 2}^{2}$, and, to a lesser degree, $R_{o s, 2}^{2}$. Instead of attempting a more sophisticated parameterization, we simply note this fact, and would recommend that an experimental estimation of the source deformation $\epsilon$ is probably best extracted from $R_{s, 2}^{2} / R_{s, 0}^{2}$, which should be unaffected by azimuthal structure of the temporal scale. From the study (below) of parameter variations in the blast-wave, the approxi- 


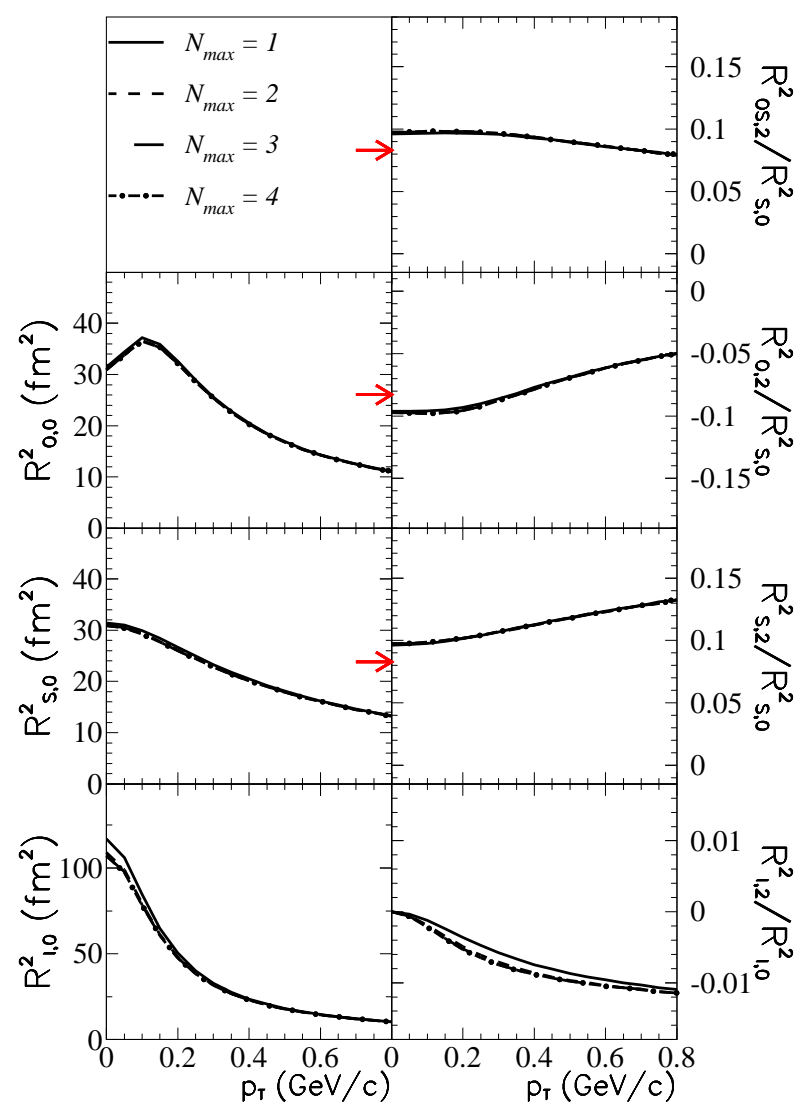

FIG. 24: (Color online) Fourier coefficients of the $\phi_{p^{-}}$ dependence of the squared HBT radii, as calculated by Equation 29] using several values of $N$, the maximum value of $n$ taken in the summation of Equation 8 Values of the blastwave parameters are for a "non-round" source, as listed in Table【 Arrows indicate values calculated from Equation 32 See text for details.

mation $\epsilon \approx 2 R_{s, 2}^{2} / R_{s, 0}^{2}$ is good to $\sim 30 \%$, for RHIC-type sources.

Figure 25] also shows results from the "IPES" hydrodynamic calculation. Here, the freeze-out shape is extended in-plane 23], but dynamical effects are so strong in this extreme case, that even the sign of the transverse radius oscillations changes with $p_{T}$. The relationships in Equation 32 work only at low $p_{T}$, and even there only very approximately. According to this model, then, geometrical considerations dominate the HBT radius oscillations, while dynamical effects begin to dominate at much higher energies.

In Figure [26] the source geometry is azimuthally isotropic $\left(R_{x}=R_{y}=12.042 \mathrm{fm}\right)$, while the flow field is varied from having a stronger boost in-plane $\left(\rho_{2}>0\right)$ to a stronger boost out-of-plane $\left(\rho_{2}<0\right)$. We notice again that the average HBT radius values $R_{\mu, 0}^{2}$ are unaffected by the anisotropy. The oscillations $\left(R_{\mu, 2}^{2}\right)$ are driven by flow gradients. Naively, one would expect that

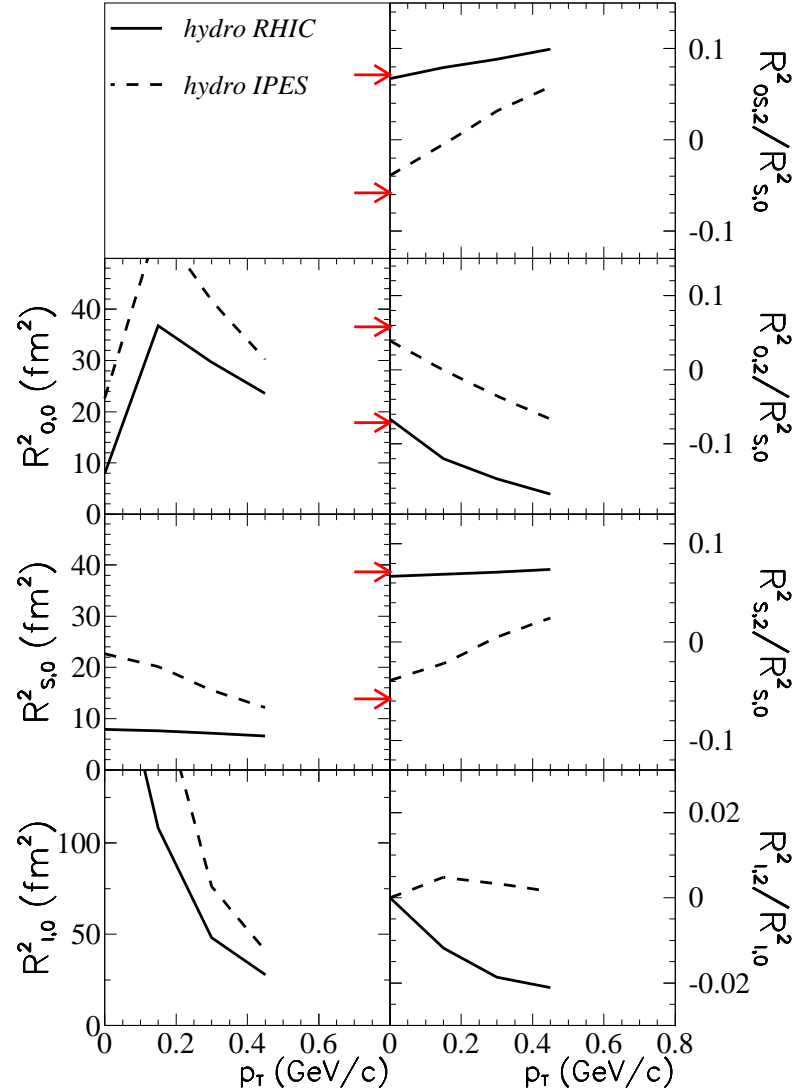

FIG. 25: (Color online) Fourier coefficients of the $\phi_{p^{-}}$ dependence of the squared HBT radii, as calculated by Equation [29] The data are not from blast-wave calculations, but from hydrodynamic calculations of Heinz and Kolb [23], for two different equations of state and initial conditions. Arrows indicate values calculated from Equation 32 and extracted values of freezeout edge radii; see text for details.

all HBT radii $R_{o}, R_{s}, R_{l}$ would be smaller when the emission angle $\phi_{p}$ is in the direction of the strongest boost. For $\rho_{2}=0.05$, for example, the radii would be smaller at $\phi_{p}=0$, the direction of stronger transverse boost; this would correspond to $R_{\mu, 2}^{2}<0(\mu=o, s, l)$. For $\mu=o$ and $\mu=l$, this is indeed observed, at all $p_{T} . R_{s, 2}^{2}$, however, changes sign from positive at low $p_{T}$, to negative at high $p_{T}$. This behaviour at low $p_{T}$ is due to an effect similar to that which led to negative proton $v_{2}$ at low $p_{T}$, even when $\rho_{2}>0$. (See discussion surrounding Figure 12]) In the present case, the particles with $p_{T} \approx 0$ are more likely to be emitted by source elements positioned along the $y$-axis, due to the strong in-plane boost for source elements with large spatial coordinate $x$. The homogeneity region for $p_{T}=0$ particles is independent of $\phi_{p}$, and has larger extent out-of-plane than in-plane. Thus we find the counter-intuitive result that $R_{s}^{2}\left(\phi_{p}=0\right)>R_{s}^{2}\left(\phi_{p}=\frac{\pi}{2}\right) \rightarrow R_{s, 2}^{2}>0$ at $p_{T} \approx 0$. We note that a similar argument holds for $R_{o}^{2}$, except that it 


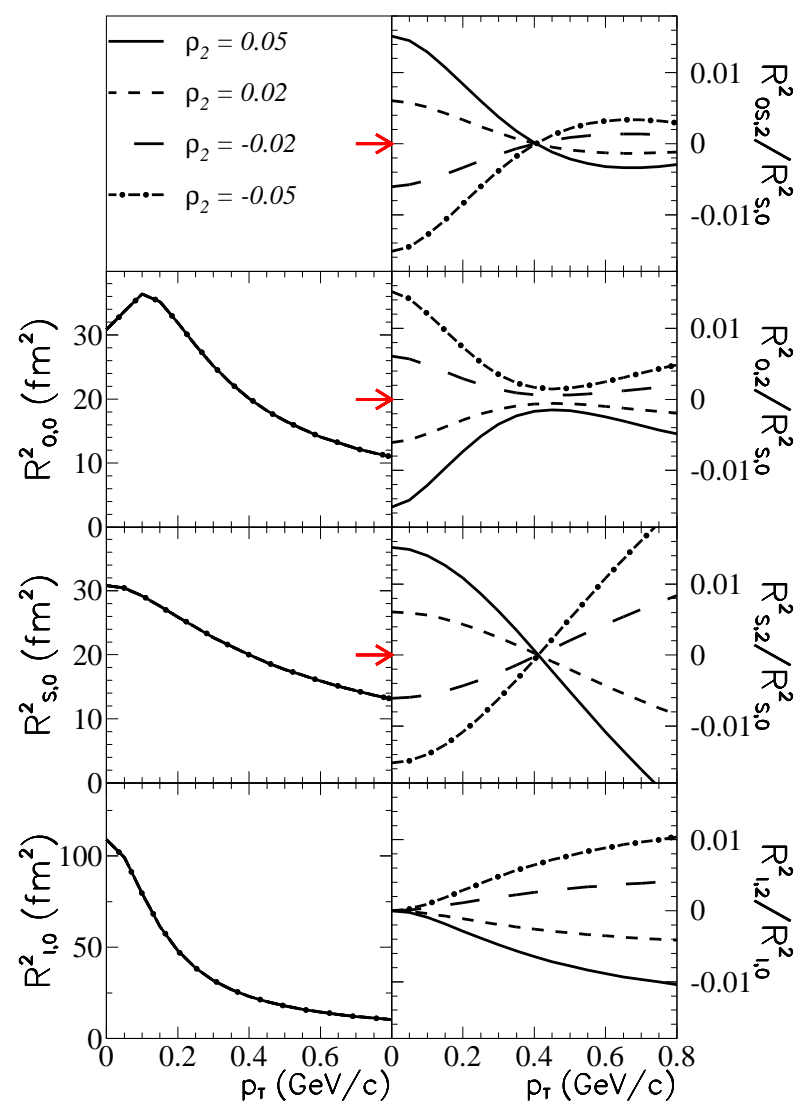

FIG. 26: (Color online) Fourier coefficients of the $\phi_{p^{-}}$ dependence of the squared HBT radii, as calculated by Equation 29] The anisotropy of the flow field $\left(\rho_{2}\right)$ is varied. Values of the other parameters are for a "round" source, as listed in Table【 Arrows indicate values calculated from Equation 32 see text for details.

leads to the conclusion that $R_{o, 2}^{2}<0$ at $p_{T} \approx 0$, and so goes in the same direction as flow-gradient effects. It is only for $R_{s}^{2}$ that the two effects compete.

Finally, comparing the scales on the right-hand panels of Figures 23] and 26] it is clear that, while the $2^{\text {nd }}$-order coefficients are driven by both anisotropic geometry and flow field, a variation in geometry $\left(R_{y} / R_{x}\right)$ has a stronger effect on $R_{\mu, 2}^{2}$ than a variation in $\rho_{2}$, when these parameters are varied by amounts which would generate a similar effect on elliptic flow $v_{2}$ (cf Figures 14 and 16). Thus, measurement of both $v_{2}$ and HBT radius oscillations would allow independent determination of both anisotropic flow strength $\rho_{2}$ and shape $R_{y} / R_{x}$.

In Figure 27 we consider anisotropy simultaneously in both the source geometry and the flow field. The source is extended out-of-plane $\left(R_{y}=13 \mathrm{fm}\right.$ and $\left.R_{x}=11 \mathrm{fm}\right)$, and the flow anisotropy $\left(\rho_{2}\right)$ varied. The $0^{\text {th }}$-order coefficients remain unaffected by the anisotropies, while the $R_{\mu, 2}^{2}$ reflect essentially the cumulative effects of the geometric anisotropy (shown in Figure 231) and the flow field

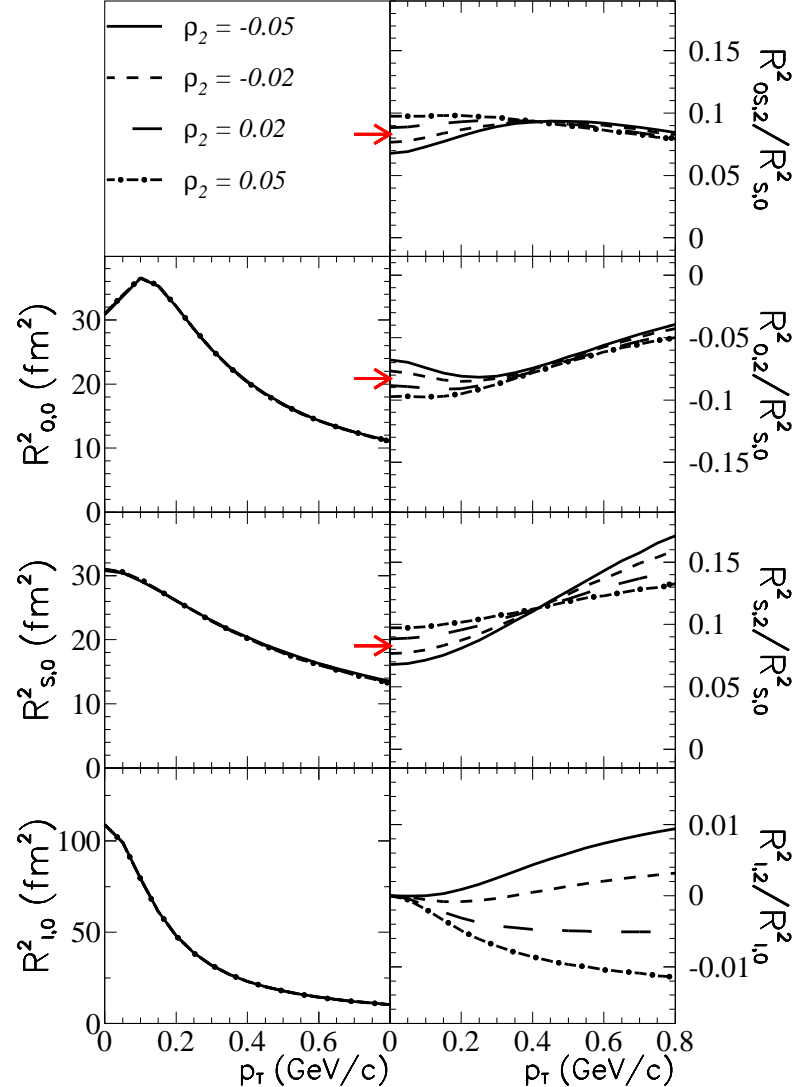

FIG. 27: (Color online) Fourier coefficients of the $\phi_{p^{-}}$ dependence of the squared HBT radii, as calculated by Equation 29] The anisotropy of the flow field $\left(\rho_{2}\right)$ is varied. Values of the other parameters are for a "non-round" source, as listed in Table I Arrows indicate values calculated from Equation 32 see text for details.

anisotropy (shown in Figure 26), with no strong nonlinear coupling between them. Thus, while in principle anisotropic flow effects may mask or dominate geometric anisotropy [9], flow field anisotropies represent small perturbations on the dominant geometric effects in the blast-wave, using "realistic" (cf Section 4) parameters.

In Figure 28 we show the effect of increasing the transverse size of the source $\left(\sqrt{R_{y}^{2}+R_{x}^{2}}\right)$ while keeping the shape $\left(R_{y} / R_{x}\right)$ and other source parameters fixed. As expected, the purely-spatial transverse radius $R_{s}^{2}$ (average and oscillation amplitude) increases proportionally with $R_{y}^{2}+R_{x}^{2}$. The squared "outward" radius parameter, $R_{o}^{2}$, contains both spatial components (which increase with $R_{y}^{2}+R_{x}^{2}$ ) and temporal components (which do not). Thus, its average value, $R_{o, 0}^{2}$, increases almost proportional to $R_{y}^{2}+R_{x}^{2}$ at low $p_{T}\left(\beta_{\perp} \sim 0\right)$ and less so at higher $p_{T}$. Due to the near-cancellation of $\phi_{p}$-dependence of temporal terms, the increase in oscillation amplitudes $R_{o s, 2}^{2}$ and $R_{o s, 2}^{2}$ is driven mainly by the spatial terms, so 


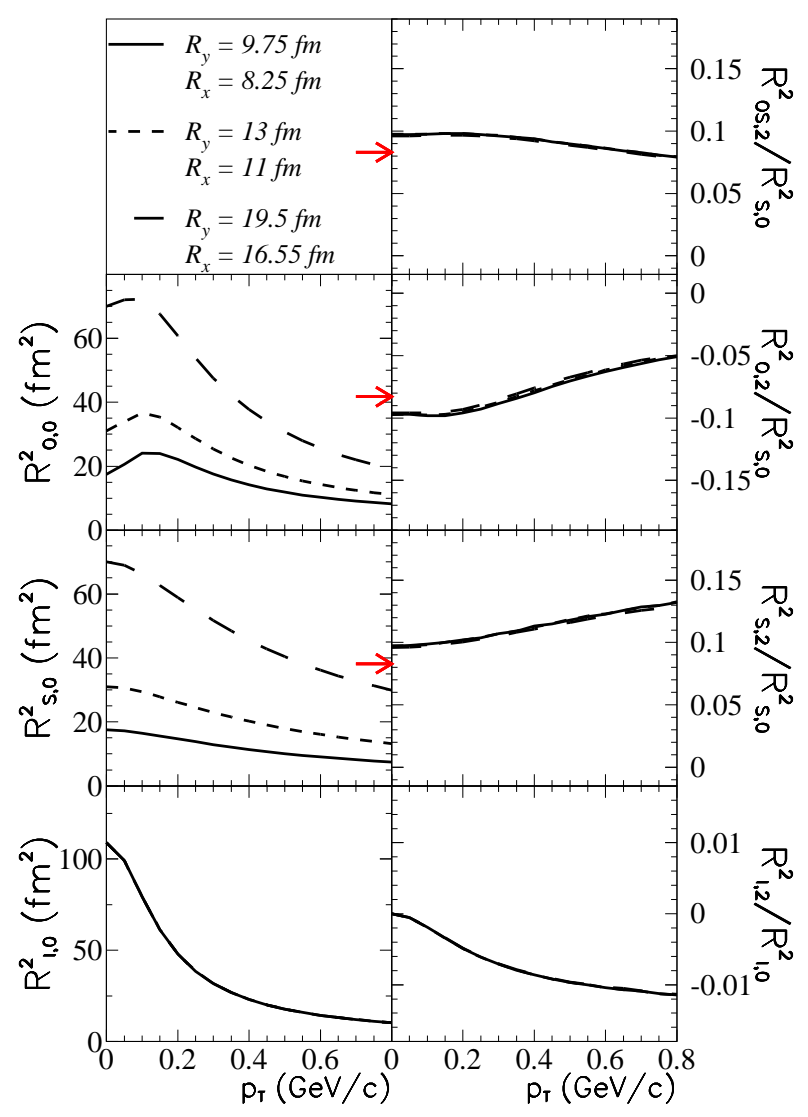

FIG. 28: (Color online) Fourier coefficients of the $\phi_{p^{-}}$ dependence of the squared HBT radii, as calculated by Equation 29] The overall transverse scale $\left(\sqrt{R_{y}^{2}+R_{x}^{2}}\right)$ is varied, while keeping $R_{y} / R_{x}=13 / 11$. Values of the other parameters are for a "non-round" source, as listed in Table【 Arrows indicate values calculated from Equation 32 see text for details.

that $R_{o, 2}^{2} / R_{s, 0}^{2}$ and $R_{o s, 2}^{2} / R_{s, 0}^{2}$ display almost no sensitivity to $R_{y}^{2}+R_{x}^{2}$ at any $p_{T}$. The longitudinal radius $R_{l}^{2}$ is unaffected by variation in the transverse scale.

In Figures [29] and [30] we vary the timescale parameters $\tau_{0}$ and $\Delta \tau$, respectively. All dependence of $R_{\mu, n}^{2}$ on these parameters come directly through the dependence of $\left\langle\tilde{x}_{\mu} \tilde{x}_{\nu}\right\rangle$, which are listed explicitly in Equations 26 After an inspection of those equations, it is unsurprising that the effects of varying these parameters are similar. The space-time correlation coefficients which depend on the timescale parameters are $\langle\tilde{x} \tilde{t}\rangle,\langle\tilde{y} \tilde{t}\rangle,\left\langle\tilde{t}^{2}\right\rangle$, and $\left\langle\tilde{z}^{2}\right\rangle$. According to Equation 22 then, $R_{s, 0}^{2}$ and $R_{s, 2}^{2}$ are unaffected by variations in $\tau_{0}$ and $\Delta \tau . R_{l}^{2}=\left\langle\tilde{t}^{2}\right\rangle$ is directly proportional to $\left(3 \Delta \tau^{2}+\tau_{0}^{2}\right)$, so $R_{l, 0}^{2}$ and $R_{l, 2}^{2}$ both scale with that quantity. Turning to the HBT radii with both spatial and temporal contributions, we again find that the $\phi_{p}$-dependence of the temporal terms is negligible, so that $R_{o, 2}^{2} / R_{s, 0}^{2}$ and $R_{o s, 2}^{2} / R_{s, 0}^{2}$ are independent of the timescales, while $R_{o, 0}^{2}$ displays the well-known [6] sensi-

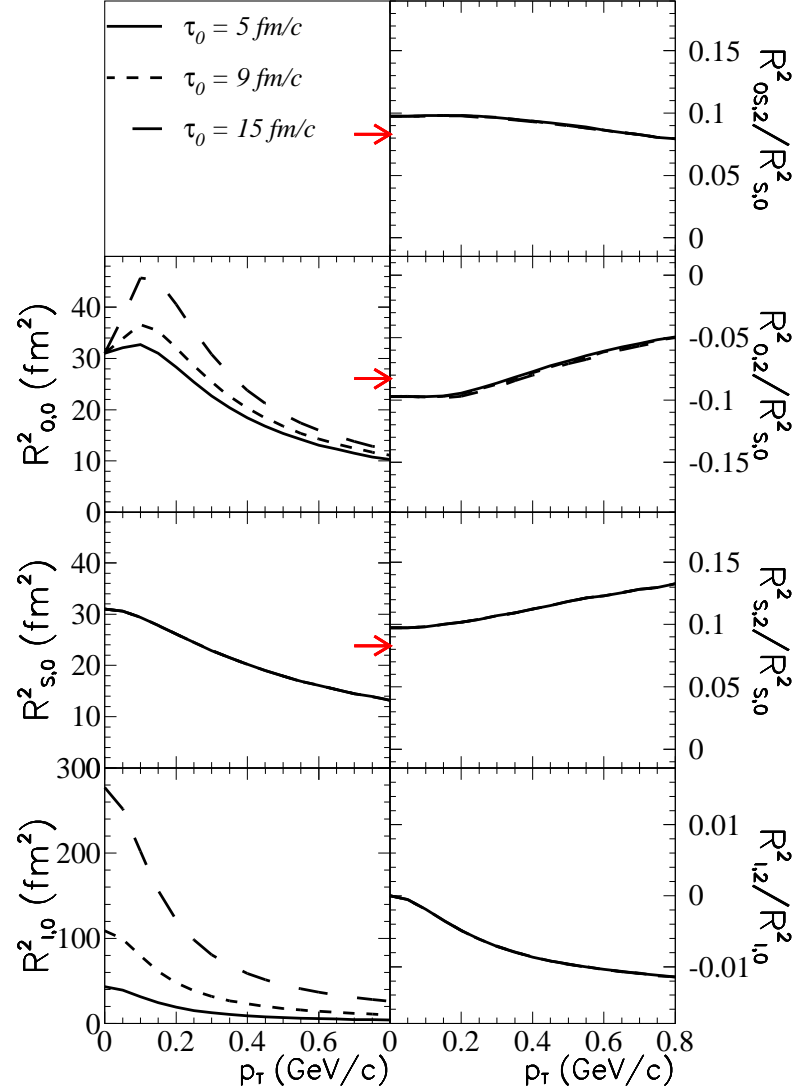

FIG. 29: (Color online) Fourier coefficients of the $\phi_{p^{-}}$ dependence of the squared HBT radii, as calculated by Equation 29] The evolution duration $\tau_{0}$ is varied. Values of the other parameters are for a "non-round" source, as listed in Table【 Arrows indicate values calculated from Equation 32 see text for details.

tivity to timescale.

Thus, we find that, in the blast-wave parameterization, essentially all sensitivity to timescales comes through the $\phi_{p}$-independent quantities $R_{o, 0}^{2}$ and $R_{l, 0}^{2}$. However, it is important to point out that an experimental estimate of the freeze-out geometric anisotropy, defined in Equation 32 from measurements of the $R_{\mu, n}^{2}$ would place an additional constraint on the evolution timescale $\tau_{0}$. In particular, the large initial-state anisotropy in coordinate space $\left(R_{y}>R_{x}\right)$ in a non-central collision will be reduced due to stronger flow in-plane than out-of-plane $\left(\rho_{2}>0\right.$ in the present parameterization). If the source lives for a long time (large $\tau_{0}$ ), the system may become round $\left(R_{y}=R_{x}\right)$ or even in-plane extended $\left(R_{y}<R_{x}\right)$ [24]. A quantitative constraint on $\tau_{0}$ from the relationship of the initial to freeze-out anisotropies, however, must be made in the context of a realistic dynamical model and is beyond the scope of the blast-wave parameterization.

In Figure 31 the effect of variations in the $\phi$-averaged ("radial") flow on the HBT radius parameters is shown. 


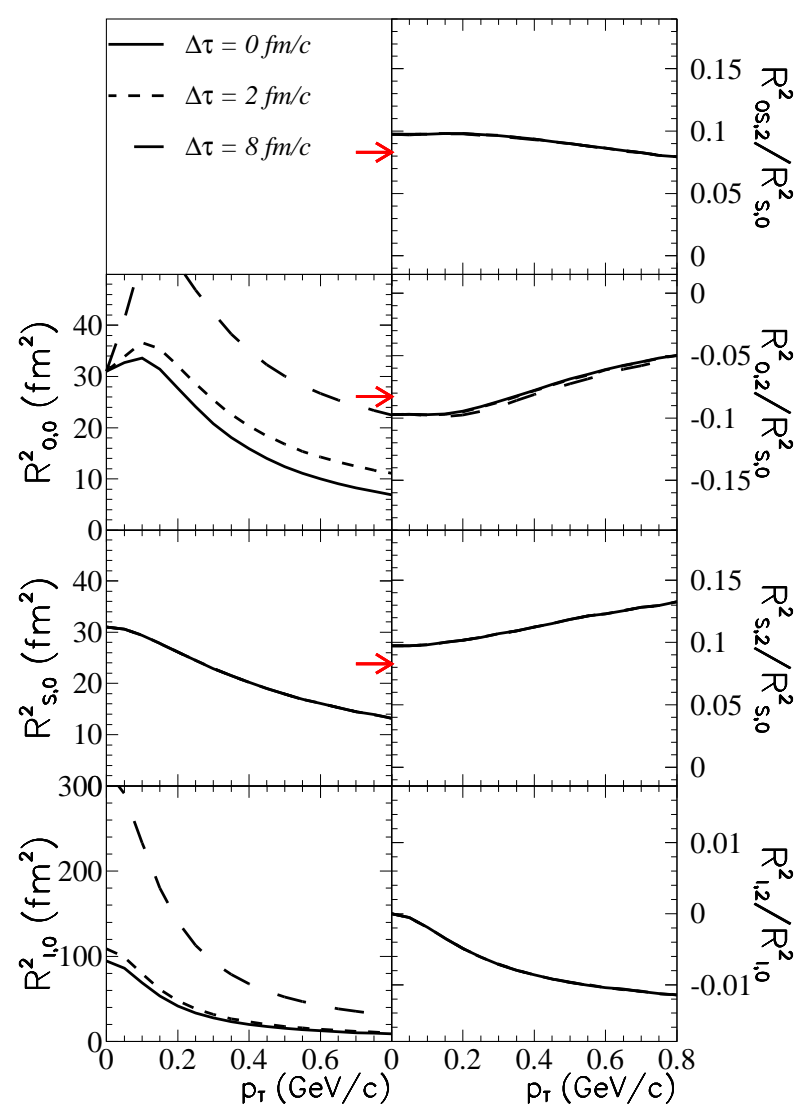

FIG. 30: (Color online) Fourier coefficients of the $\phi_{p^{-}}$ dependence of the squared HBT radii, as calculated by Equation 29] The evolution duration $\Delta \tau$ is varied. Values of the other parameters are for a "non-round" source, as listed in Table【 Arrows indicate values calculated from Equation 32 see text for details.

As is well-known [] , stronger flow reduces the homogeneity lengths, and, indeed, almost all of the $\left|R_{\mu, n}^{2}\right|$ fall with increasing $\rho_{0}$. The one interesting exception is $R_{l, 2}^{2}$; while the average scale of the longitudinal radius $\left(R_{l, 0}^{2}\right)$ decreases as the flow is increased, its $\phi_{p}$-dependence $\left(\left|R_{l, 2}^{2}\right|\right)$ increases (and so, then, does $\left.\left|R_{l, 2}^{2} / R_{l, 0}^{2}\right|\right)$. This is because there is no explicit $\phi_{p}$ dependence in $R_{l}^{2}=\left\langle\tilde{z}^{2}\right\rangle$; any $\phi_{p}$ dependence is implicit, and thus is generated by spacemomentum correlations 44, which, in this model, arise solely from flow. In the no-flow limit for a boost-invariant source, $\left\langle\tilde{t}^{2}\right\rangle,\left\langle\tilde{x}^{2}\right\rangle,\left\langle\tilde{y}^{2}\right\rangle$, and $\left\langle\tilde{z}^{2}\right\rangle$ are all $\phi_{p}$-independent constants [44, 47, 48].

Indeed, it is for a similar reason that $R_{l, 2}^{2}$ vanishes for $p_{T}=0$, independent of model parameter in Figures 23 . [32 At $p_{T}=0$, symmetry demands that none of the spatial correlation coefficients $\left\langle\tilde{x}_{\mu} \tilde{x}_{\nu}\right\rangle$ may depend on $\phi_{p}$. Hence, only HBT radii with explicit $\phi_{p}$-dependence may exhibit an oscillation in that limit.

Finally, we note in Figure 31 that the oscillation

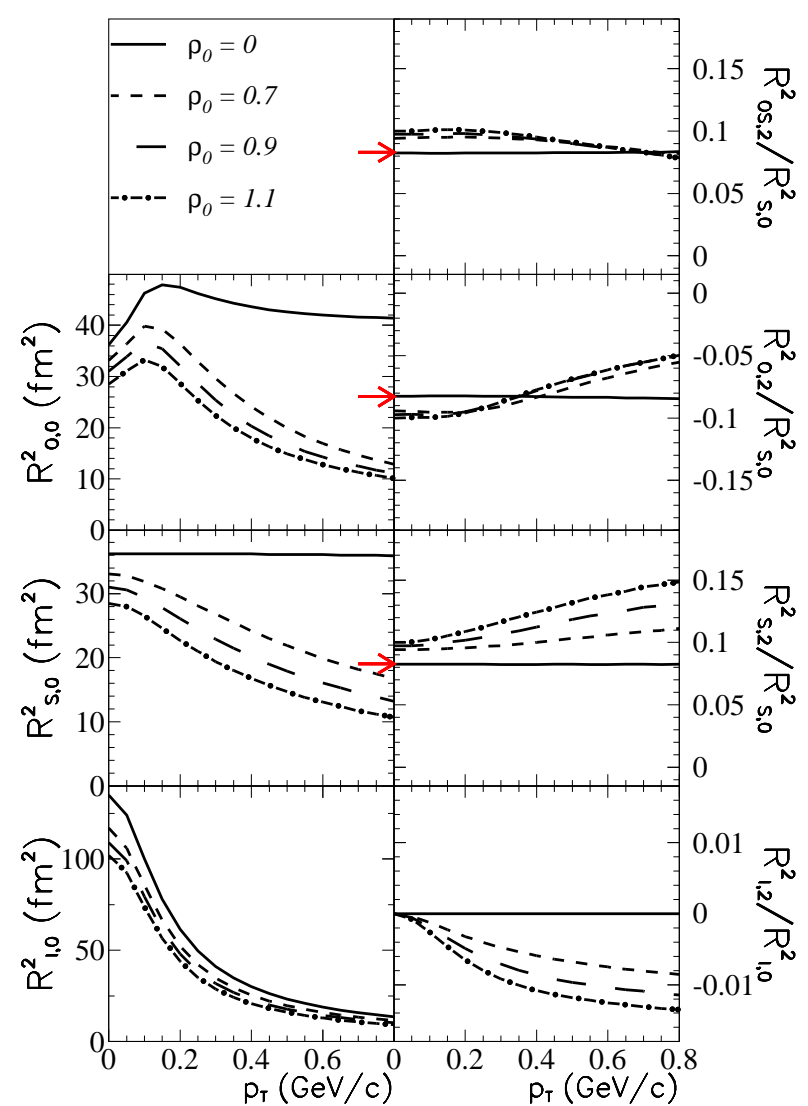

FIG. 31: (Color online) Fourier coefficients of the $\phi_{p^{-}}$ dependence of the squared HBT radii, as calculated by Equation 29] The average radial flow magnitude $\rho_{0}$ is varied. Values of the other parameters are for a "non-round" source, as listed in Table I Arrows indicate values calculated from Equation 32 see text for details.

strengths $\left|R_{o, 2}^{2}\right|$ and $\left|R_{s, 2}^{2}\right|$ are somewhat less diminished (at low $p_{T}$ ) by increasing radial flow, than is $R_{s, 0}^{2}$, which measures the overall spatial scale of the homogeneity region. Here, we offer no simple insights on the interplay between the increasing deformation of the homogeneity region, and its decreasing scale, but simply note that the dependence of the scaled oscillation strengths on $\rho_{0}$ is rather small, especially at low $p_{T}$, even for the unrealistic case of no average transverse flow $\left(\rho_{0}=0\right)$.

Figure 32 explores the effect on HBT radii of varying the temperature parameter $T$. Increasing $T$ increases "thermal smearing", reducing the flow-induced spacemomentum correlations. It is well known that this leads to increased homogeneity lengths and HBT radii [36], as reflected in the left panels of the Figure. In the present model, we find a small residual dependence of $R_{\mu, 2}^{2}$ on $T$ beyond the scaling of $R_{\mu, 0}^{2}$ (right panels).

In the limit of very high temperature, all spacemomentum correlations are eliminated, and all oscillations of HBT radii are again due to the explicit $\phi_{p^{-}}$ 


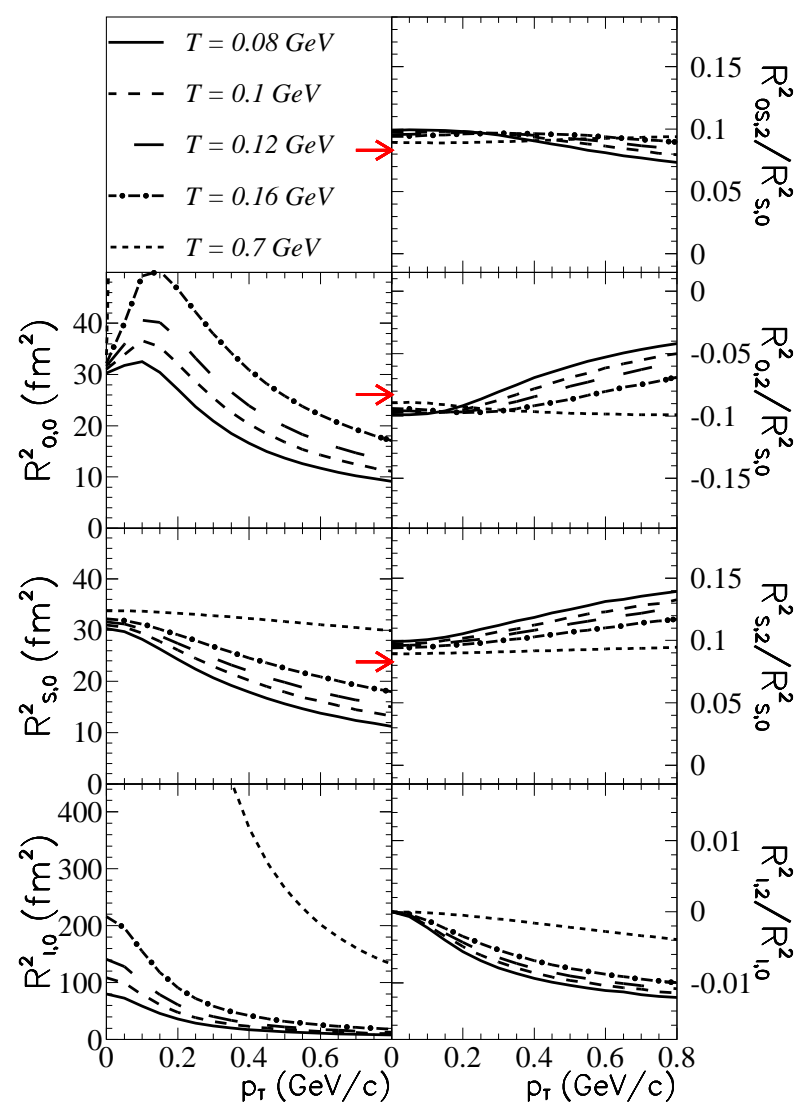

FIG. 32: (Color online) Fourier coefficients of the $\phi_{p^{-}}$ dependence of the squared HBT radii, as calculated by Equation 29 For $T=0.7 \mathrm{GeV} / \mathrm{c}, R_{o, 0}^{2}$ and $R_{l, 0}^{2}$ curves partially exceed the displayed scale. Values of the other parameters are for a "non-round" source, as listed in Table【 Arrows indicate values calculated from Equation 32 see text for details.

dependence in Equations 22. Then, we find that Equations 30,32 hold, independent of $p_{T}$. For the source of Figure 32 according to Equation $32\left|R_{\mu, 2}^{2} / R_{s, 0}^{2}\right|=0.083$. Finally, $R_{l, 2}^{2}$ decreases with increasing $T$, and must vanish at $T \rightarrow \infty$, when all space-momentum correlations are destroyed.

Finally, we consider the effect of a finite "skin thickness" $a_{s}$. As in the discussions surrounding Figures $\mathbf{7}$ and 17 it is appropriate to scale the flow parameters according to $F\left(a_{s}\right)$ given in Equation 20] Moreover, it is clearly appropriate to scale the geometric size parameters $R_{y}$ and $R_{x}$, as the overall scale of the source will increase with $a_{s}$, if these parameters remain fixed. Less clear is the exact scaling which would keep, e.g. $R_{s, 0}^{2}$, independent of $a_{s}$, especially in the presence of finite flow. For illustrative purposes, we scale $R_{y}$ and $R_{x}$ also by $F\left(a_{s}\right)$, so that $R_{y}=13.0,12.21$, and $8.84 \mathrm{fm}$, for $a_{s}=0,0.1$, and 0.3 , respectively; $R_{y} / R_{x}=13 / 11$ in all cases. This scaling keeps $\langle|r|\rangle$, as well as the flow gradient, the same in each case.

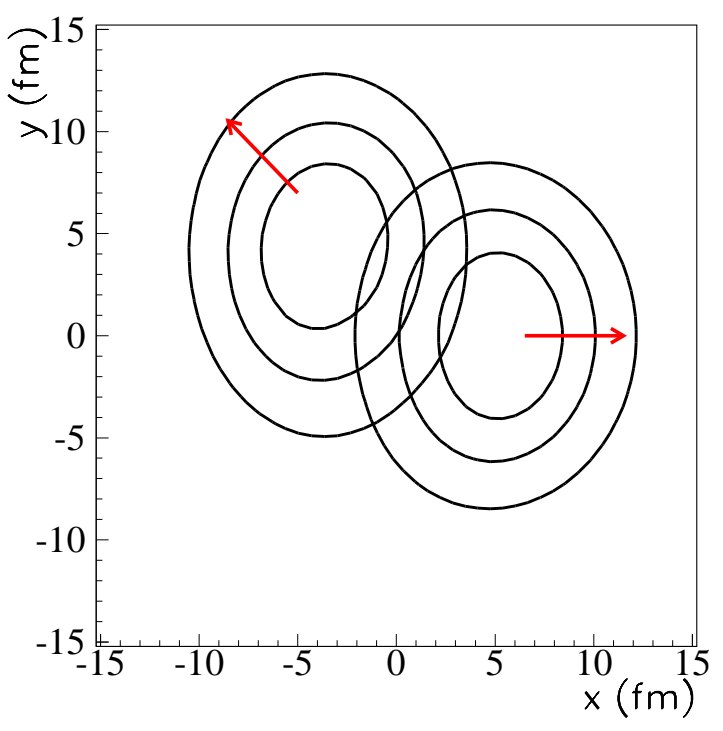

FIG. 33: (Color online) Emission probability contours, plotted on a linear scale, indicate emission zones for pions with $p_{T}=0.3 \mathrm{GeV} / \mathrm{c}$ at $\phi_{p}=0^{\circ}$ and $\phi_{p}=135^{\circ}$ (indicated by arrows), from a blast-wave source with $a_{s}=0.3$. Other parameter values correspond to the "non-round" values listed in Table 凹

Figure 33 shows homogeneity regions projected onto the $x-y$ plane, for a blast-wave source with $a_{s}=0.3$, corresponding to a pseudo-Gaussian geometrical distribution in $\tilde{r}$ (see Figure 11). Comparing Figures 18 and 33. we observe that the lack of a "hard cut-off" in coordinate space in the latter case leads to less reduction in the "out" direction (i.e. along the direction of motion). Thus, the important ratio $R_{o, 0}^{2} / R_{s, 0}^{2}$ will be larger at finite $p_{T}$, when $a_{s}=0.3$.

We also note that the shape and size of the homogeneity region itself depends much less on $\phi_{p}$, when $a_{s}=0.3$. The homogeneity region for pions emitted to $\phi_{p}=135^{\circ}$ is essentially a spatially-translated (and unrotated) replica of that for pions emitted to $\phi_{p}=0^{\circ}$. Since HBT correlations are insensitive to a spatial translation of the source, the situation for $a_{s}=0.3$ is rather similar to the situation in which no flow is present. In the no-flow case, the same homogeneity region is measured at all angles, and all oscillations of the HBT radii arise from the explicit $\phi_{p}$ dependence in Equations 22 [44, 47]; for the source in Figure 33 not the same region, but a (virtually) identical one, is being measured at all angles $\phi_{p}$.

This has implications for the oscillations of the transverse radii. Focusing on the homogeneity regions for $\phi_{p}=135^{\circ}$, we observe that while $\langle\tilde{x} \tilde{y}\rangle$ (which quantifies the "tilt" of the homogeneity region relative to the $x$ and $y$ axes) is greater when $a_{s}=0, R_{o s}^{2}$ (which quantifies the tilt of the homogeneity region relative to the "out" and "side" directions 23 ) is larger for $a_{s}=0.3$ Therefore, we expect larger values of $R_{o s, 2}^{2}$ for larger $a_{s}$. 


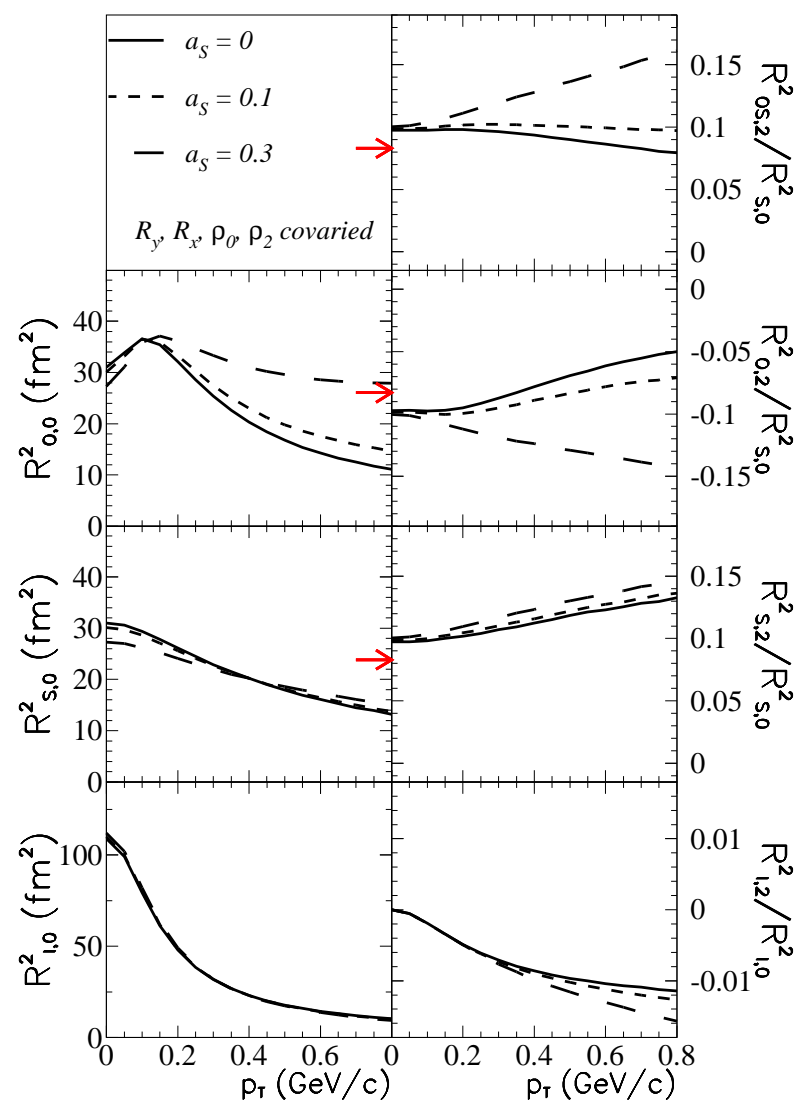

FIG. 34: (Color online) Fourier coefficients of the $\phi_{p^{-}}$ dependence of the squared HBT radii, as calculated by Equation 29 The parameters $T, \tau_{0}$ and $\Delta \tau$ are set to values listed in Table \ while the parameters $\rho_{0}, \rho_{2}, R_{y}$ and $R_{x}$ are covaried with the surface diffuseness $a_{s}$; see text for details.

Figure 34 shows quantitatively the effects on the HBT radii, when $a_{s}$ is varied. As intended by the scaling of flow and size parameters with $F\left(a_{s}\right), R_{s, 0}^{2}$ remains approximately invariant when the parameters are covaried. As discussed above, $R_{o, 0}^{2} / R_{s, 0}^{2}$ grows with $a_{s}$ at finite $p_{T}$, as do the magnitudes of the oscillation strengths $\left|R_{o, 2}^{2} / R_{s, 0}^{2}\right|,\left|R_{o s, 2}^{2} / R_{s, 0}^{2}\right|$ and $\left|R_{s, 2}^{2} / R_{s, 0}^{2}\right|$. The average value of $R_{l}^{2}$ remains roughly constant, while its oscillation amplitude increases slightly with $a_{s}$, due to $x-z$ and $y-z$ correlations.

\section{Non-identical particle correlations}

Final-state interactions between pairs of non-identical particles (e.g. $K-\pi$ ) are sensitive to the space-time structure (size, shape, emission duration) of the emitting source, analogously to HBT correlations between identical particles. Most importantly, the authors of Ref. [69, 70, 71] show that studying correlations between non-identical particles reveals new information about the average relative space-time separation between the emission of the two particles, in the rest frame of the pair. This unique information may be extremely valuable to determine the interplay between partonic and hadronic effects. For example, the lower hadronic cross-section of kaons compared to pions may cause them to be emitted earlier and closer to the center of the source than pions. However, if most of the system evolution takes place at the parton level, the space-time emission pattern of pions and kaons would be similar. This example is far from unique as the same kind of argument can be made for protons recalling that pion-proton hadronic cross section are very large, or conversely to $\Xi$ and $\Omega$ whose hadronic cross section is expected to be small. Furthermore, in terms of temporal effects, strangeness distillation 72, 73] or other unique physics may cause some particles to be emitted later than others. Preliminary analyses of the $\pi^{ \pm}-K^{ \pm}$, $\pi^{ \pm}-p^{ \pm}, K^{ \pm}-p^{ \pm}$correlation functions have been reported by the STAR collaboration in Au-Au collisions at $\sqrt{s_{N N}}=130 \mathrm{GeV}$ and $200 \mathrm{GeV}[74,75]$. These analyses show that pions, kaons and protons are not emitted at the same average space-time point.

The blast-wave parameterization implicitly assumes that the particle freeze-out conditions (temperature, flow profile, freeze-out time and position) are the same for all particle species. However, as shown earlier, the transverse momentum spectra and elliptic flow or different particle species do not look alike. Indeed, the relative contribution of the random emission (quantified by temperature) and collective expansion depends directly on particle masses. The same phenomena is likely to affect the particle freeze-out space-time emission distribution. In this section, we will show that collective flow effects implicit in the blast-wave parameterization induce a shift between the average freeze-out space-time point of different particle species. We will then study how changing the blast-wave parameters affects these average freezeout separations.

\section{Non-identical particle and blast-wave formalism}

Two particles interact when they are close to each other in phase space in the local pair rest frame. Thus, particle pairs may be correlated when their relative momentum in the pair rest frame is small. For particles with different masses, a small relative momentum in the pair rest frame means that both particles have similar velocities in the laboratory frame and not similar momentum. This point is particularly important to realize when studying correlation involving pions. Due to the low pion mass, the pion velocity is the same as heavier particles (e.g. kaons or protons) when its momentum is much lower than the particle momentum it is associated with. For example, a proton with a momentum of 1 $\mathrm{GeV} / \mathrm{c}$ has the same velocity as a pion with a momentum of $\approx 0.15 \mathrm{GeV} / \mathrm{c}$.

As described in Ref. 76], the spatial separation be- 
tween particles in the pair rest frame can be projected along three axes, $\left\langle r_{\text {out }}^{*}\right\rangle$, along the pair transverse momentum, $\left\langle r_{\text {side }}^{*}\right\rangle$, perpendicular to the pair transverse momentum and $\left\langle r_{\text {long }}^{*}\right\rangle$, along the beam axis. To study the blast-wave prediction, we focus on the limiting case where the relative momentum between both particles in the pair rest frame is zero, which means that both particles have the same velocity. Thus we calculate the separation between particle 1 and 2 in the pair rest frame $\left\langle\Delta r_{\text {out }}^{*}\right\rangle$, $\left\langle\Delta r_{\text {side }}^{*}\right\rangle$ and $\left\langle\Delta r_{\text {long }}^{*}\right\rangle$, at a given pair transverse velocity $\beta_{T}$, azimuthal angle, $\phi_{p}$, and longitudinal velocity, $\beta_{L}$ :

$$
\begin{aligned}
\left\langle\Delta r_{\text {out }}^{*}\right\rangle & =\left\langle r_{\text {out }}^{*}(1)\right\rangle-\left\langle r_{\text {out }}^{*}(2)\right\rangle \\
\left\langle\Delta r_{\text {side }}^{*}\right\rangle & =\left\langle r_{\text {side }}^{*}(1)\right\rangle-\left\langle r_{\text {side }}^{*}(2)\right\rangle \\
\left\langle\Delta r_{\text {long }}^{*}\right\rangle & =\left\langle r_{\text {long }}^{*}(1)\right\rangle-\left\langle r_{\text {long }}^{*}(2)\right\rangle
\end{aligned}
$$

With the particle emission points defined as $(x, y, z, t)$ :

$$
\begin{aligned}
\left\langle r_{\text {out }}^{*}\right\rangle & =\gamma_{T}\left(\langle x\rangle \cos \left(\phi_{p}\right)+\langle y\rangle \sin \left(\phi_{p}\right)-\beta_{T}\langle t\rangle\right) \\
\left\langle r_{\text {side }}^{*}\right\rangle & =\langle y\rangle \cos \left(\phi_{p}\right)-\langle x\rangle \sin \left(\phi_{p}\right) \\
\left\langle r_{\text {long }}^{*}\right\rangle & =\gamma_{L}\left\langle(z)-\beta_{L}(t)\right\rangle
\end{aligned}
$$

Recalling that $\left\langle\beta_{L}\right\rangle=\langle z / t\rangle,\left\langle r_{\text {long }}^{*}(i)\right\rangle=0$. Following the notation of Equation 25 the variables $\langle x\rangle,\langle y\rangle$ and $\langle t\rangle$ depend on $\beta_{T}$ and on the particle mass (m) as:

$$
\begin{aligned}
\langle x\rangle & =\overline{\{x\}_{0,0}}\left(m, \beta_{T}\right) \\
\langle y\rangle & =\overline{\{y\}_{0,0}}\left(m, \beta_{T}\right) \\
\langle t\rangle & =\frac{\Delta \tau^{2}+\tau_{0}^{2}}{\tau_{0}} \overline{\{1\}_{0,1}}\left(m, \beta_{T}\right)
\end{aligned}
$$

The average values of $x$ and $y$ will vary with particle mass and particle transverse velocity, which yields separations between pions and kaons, pions and protons, and protons and kaons in the pair rest frame shown as in Figure [35. The dashed and dotted lines show the contribution of the spatial $\left(\Delta r_{\text {out }}\right)$ and time $(\Delta t)$ separation boosted to the pair rest frame along the pair transverse momentum, respectively. i.e. the dash line shows $\left\langle\gamma_{T} \Delta r_{\text {out }}\right\rangle$ and the dot line represents $-\left\langle\gamma_{T} \beta_{T} \Delta t\right\rangle$. The plain line shows $\left\langle\Delta r_{\text {out }}^{*}\right\rangle=\left\langle\gamma_{T}\left(\Delta r_{\text {out }}-\beta_{T} \Delta t\right)\right\rangle$, the spatial separation in the pair rest frame. When boosting to the pair rest frame, time and spatial shifts in the laboratory frame add up due to their opposite signs. The largest (smallest) shift is obtained when the mass ratio between both species is the largest (smallest), i.e. for pion-proton (kaon-proton) pairs.

In order to understand the behavior of $\Delta r_{\text {out }}^{*}$, it is instructive to investigate how the average emission points in the laboratory frame $\left(\left\langle r_{\text {out }}\right\rangle\right)$ of pions and kaons behave in various conditions as shown in Figure 36] This figure shows the average emission points of pions and kaons in four different configurations: (1) The thin plain line shows the flow profile, (Equation 4), which sets the
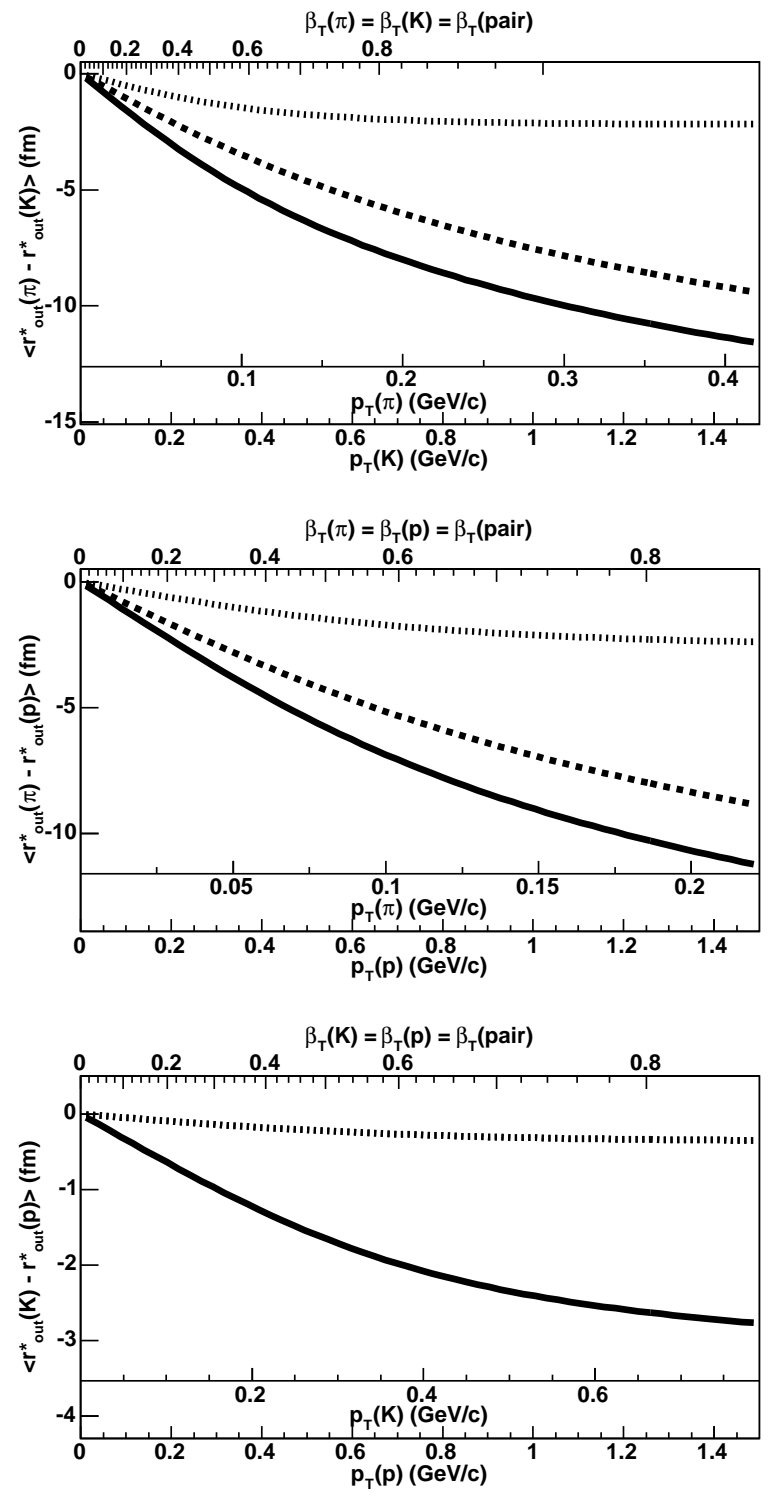

FIG. 35: Separation between the average emission point of pions, kaons and protons in the pair rest frame along the pair transverse velocity. The values of the blast-wave parameters are for a round source, as listed in Table \ Dot line: time shift in the laboratory boosted to the pair rest frame, dash line: spatial shift in the laboratory boosted to the pair rest frame, plain line: total spatial shift in the pair rest frame.

emission point of particles when $\mathrm{T}=0 \mathrm{GeV}$. (2) the dot line is calculated assuming an infinite system, i.e. $\Omega\left(r, \phi_{s}\right)=1$ and with $T=0.1 \mathrm{GeV}$. (3) the dash line corresponds to a finite system as in Equation 11 with $a_{s}=0$, and (for illustration) an extremely low temperature, $T=0.01 \mathrm{GeV}$. (4) the thick plain line is calculated using the standard parameters used in Figure 36. Only in case (1) are pions and kaons emitted exactly at the same point. Since $T=0 \mathrm{GeV}$, all particles are always emitted at the same point as set by the flow profile. In case (2), particle emission points spread around the average emis- 


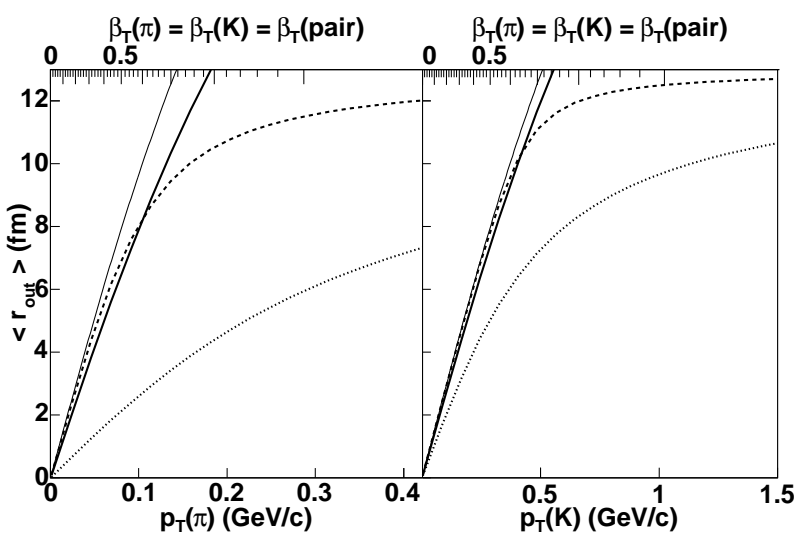

FIG. 36: Average emission points of pions (left) and kaons (right) as a function of the pion and kaon velocity and momentum. All curve are calculated with $\rho_{0}=0.9$ and $\rho_{a}=0$. The parameters $\tau$ and $\Delta t$ are irrelevant. Thin plain line: $R_{x}=R_{y}=13 \mathrm{fm}, \mathrm{T}=0 \mathrm{GeV}$. Thick plain line: infinite system (i.e. $\left.\Omega\left(r, \phi_{s}\right)=1\right)$, $\mathrm{T}=0.1 \mathrm{GeV}$. Dash line: $R_{x}=R_{y}$ $=13 \mathrm{fm}, \mathrm{T}=0.01 \mathrm{GeV}$. Dot line: $R_{x}=R_{y}=13 \mathrm{fm}, \mathrm{T}=0.1$ $\mathrm{GeV}$.

sion defined by the flow profile. At small $\eta$ there are as many particles emitted at large $r$, i.e. large $\rho$ as at small $r$. But when $|\eta|$ is large, the term $e^{-m_{T} / T \cosh \rho\left(r, \phi_{s}\right) \cosh (\eta)}$ in Equation 12 favors small $\rho$, i.e. small $r$. Thus, the average emission point $\left\langle r_{\text {out }}\right\rangle$ is smaller than the one defined by the flow profile. In case (3), the average emission points of pions and kaons follow closely the flow profile when the particle rapidity is significantly smaller than $\rho_{0}$. Close or beyond $\rho_{0}$, a certain fraction of the particle emission function is truncated due to the system finite size. The particle emission points are not allowed to spread beyond the system boundary, hence breaking the balance between particle emitted at small radii and particle emitted at large radii. Thus, the particle average emission radius is smaller than the emission radius given by the flow profile. Because the temperature is rather small, the average emission points of both species converge rapidly toward the radius of the system. The larger temperature in case (4) makes the average emission radii converge slowly toward the system limit. This phenomenon, with the addition of the phenomenon described in case (2) reduces very significantly the average particle emission radius compared to the flow profile limit.

The effect of temperature depends on particle mass and momentum. Random smearing is maximal for particles with low mass and momentum such as the low $p_{T}$ pions that are usually associated with kaons or protons in nonidentical particle correlation analyses. This effect is illustrated in Figure 37 It shows the probability of emitting pions at a given point in the transverse plane for two different pion momenta $\vec{p}=(0.15,0,0)$ and $\vec{p}=(0.3,0,0)$. Kaon and proton momenta are calculated so that they have the same velocities as pions. The region of the system that emits particles of a given momentum shrinks

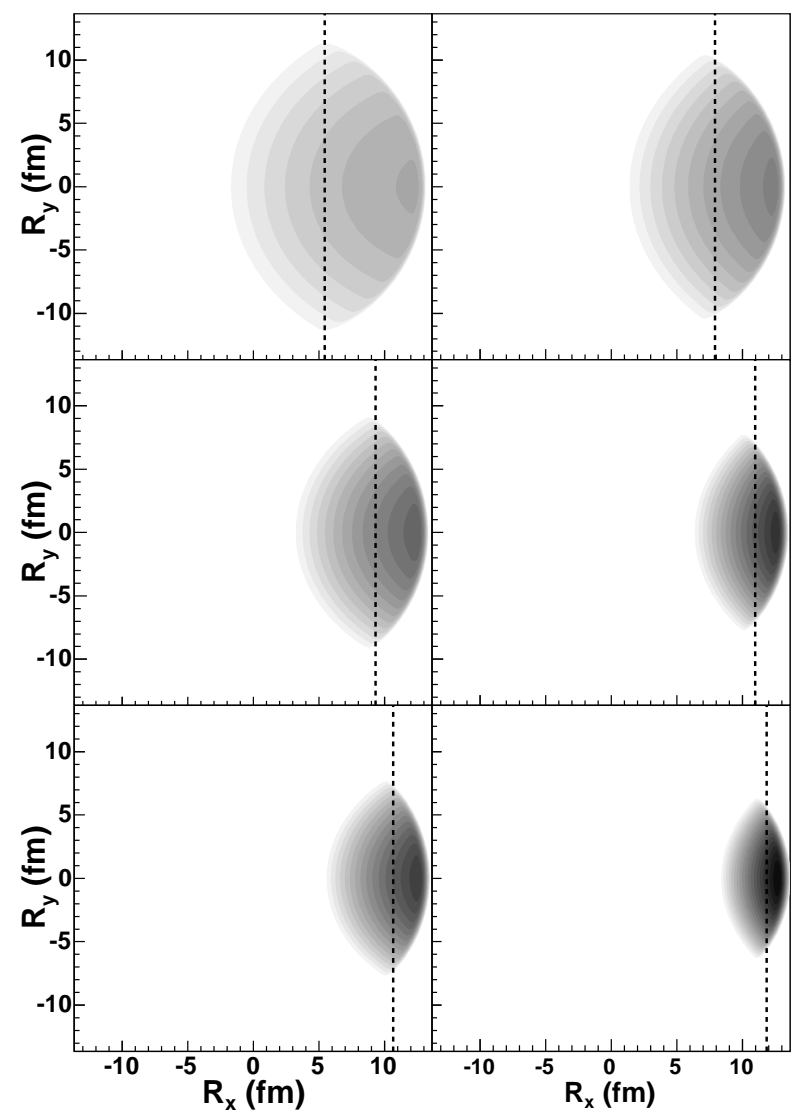

FIG. 37: Distribution of the emission points in the transverse plane, for different particle species emitted at the same velocity, $\beta_{y}=0$ and $\beta_{x}=0.907$ on the left-hand side and $\beta_{x}=$ 0.974 on the right hand side. Top panels are for pions, middle for kaons and bottom for protons. The same logarithmic color scale is used for all six panels. The blast-wave parameters are the same as in Figure 36] except that $a_{s}=0.01$ The dashed lines show $\langle x\rangle$.

and moves towards the edge of the system as the particle mass, and/or momentum increases. The magnitude of the inward radius shift depends on the fraction of the source distribution that is truncated due to the system finite size. Thus, the inward shift of the average emission radius scales with the source size. This effect yields the systematic shift between the average emission points of pions, kaons and protons as shown in Figure 35] since pion source size is the largest and proton the smallest. Light particles are emitted the closer to the center of the source than heavier ones.

In addition to a spatial separation, the blast-wave parameterization induces a time shift between different particle species emitted at the same velocity as shown by the dot line in Figure 35] Due to random motion, the space-time rapidity $(\eta)$ spreads around the momentum rapidity (Y). Because the relationship $t=\tau \cosh (\eta)$ is positive definite, the larger dispersion of $\eta$ for pions than for kaons or protons leads to a delay of the emission of pions with respect to kaons or protons. Protons are emitted 
first, then kaons and then pions. The spatial and time shifts have opposite signs in the laboratory frame but they sum up when boosting to the pair rest frame. The plain line in Figure 35 shows the added contributions of both shifts. The pion-kaon and pion-proton separation in the pair rest frame ranges from 5 to $15 \mathrm{fm}$, while the separation between kaons and protons is on the order of 2-4 fm. These shifts are large enough to be measured.

The curves on Figure 35 have been obtained by setting the blast-wave parameters to arbitrary values. We will now investigate how changing these parameters affects the shift between pions and kaons. The results obtained studying pion-kaon separation can be easily extrapolated to pion-proton and kaon-proton separations. Since experimental analyses of non-identical two-particle correlations performed to date do not investigate the azimuthal dependences with respect to the reaction plane, we will focus our study on central collisions. We will then show that the shift between the average emission points of different particle species oscillates with respect to the reaction plane without investigating the effect of varying the parameters in detail.

\section{Non-identical particle correlations in central collisions}

In central collisions, azimuthal symmetry implies that the particle emission pattern depends only on the relative angle between the position and momentum $\phi_{p}-\phi_{s}$. Thus, setting for convenience $\phi_{p}=0$, yields:

$$
\begin{aligned}
\left\langle r_{\text {out }}^{*}(i)\right\rangle & =\left\langle\gamma_{T}\left(x_{i}-\beta_{T} \Delta t_{i}\right)\right\rangle \\
\left\langle r_{\text {side }}^{*}(i)\right\rangle & =0
\end{aligned}
$$

Furthermore, we consider emission from an azimuthally-isotropic source $\left(R_{x}=R_{y}\right.$ and $\left.\rho_{2}=0\right)$ only. Hence, the only quantity of interest is $\left\langle\Delta r_{\text {out }}^{*}\right\rangle$.

Figure 38 shows the dependence of the average separation between $\pi$ and $\mathrm{K}$ in the pair rest frame as a function of the pion and kaon momentum using several values of $N$, the maximum value of $n$ taken in the summation of Equation 8 Pions and kaons are of course treated as bosons. The difference between the average separation calculated either by using a Boltzman function $(\mathrm{N}=1)$ or by approximating the Bose-Einstein distribution at the $4^{\text {th }}$ order is smaller than $0.5 \mathrm{fm}$. The maximum relative difference is on the order of $8 \%$ at small transverse momentum. The Bose-Einstein distribution already converges when $\mathrm{N}=2$.

Figure 39] shows the dependence of the spatial shift between pions and kaons in the pair rest frame as a function of the pair velocity for different temperature. The shift increases as the temperature increases all the way from $0.01 \mathrm{GeV}$ to $0.12 \mathrm{GeV}$. When the temperature is very low (e.g. $0.01 \mathrm{GeV}$ ), pion and kaon emission patterns are dominated by space-momentum correlation independent of particle masses. In the limit of zero temperature, there

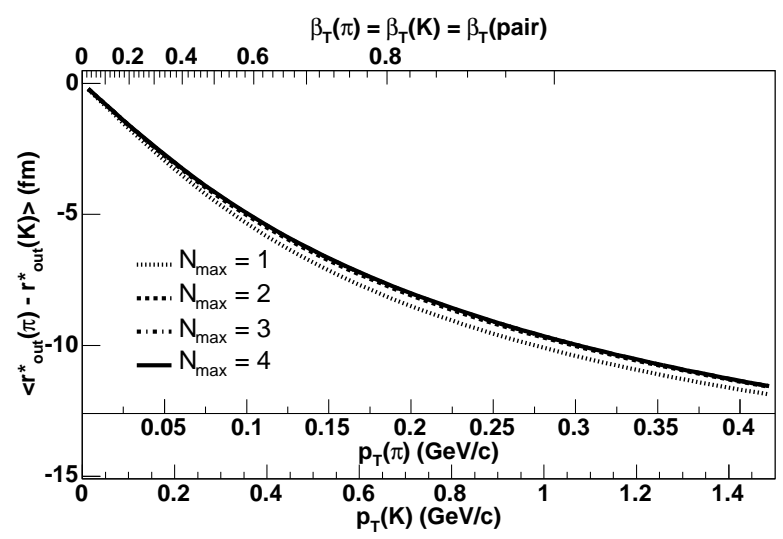

FIG. 38: Average separation between $\pi$ and $\mathrm{K}$ in the pair rest frame as a function of the pion and kaon momentum using several values of $N$, the maximum value of $n$ taken in the summation of Equation 8 Values of the blast-wave parameters are for a round source, as listed in Table $\square$

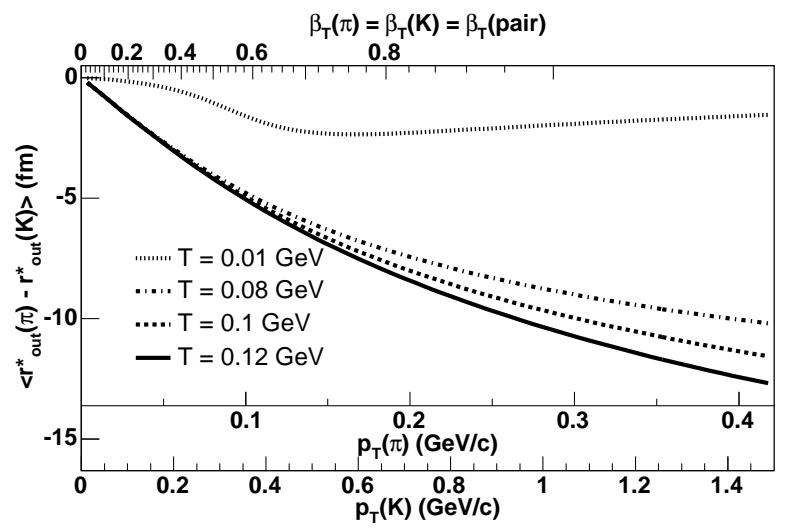

FIG. 39: Average separation between $\pi$ and $\mathrm{K}$ in the pair rest frame as a function of the pion and kaon momentum for different temperature. The values of the other blast-wave parameters are for a round source, as listed in Table

is a unique correspondence between particle velocity and emission point. In that case since we consider particles with the same velocity, pions and kaons are emitted from the same point. On the other hand when the temperature is non zero, a fraction of the pions and kaons that would be emitted in a infinite system are truncated, which shifts their average emission points inward. Because the pion source size is significantly larger than the kaon source size due to the pion lower mass and momentum, the pion average emission point is more shifted inward than kaon's, which is illustrated in Figure 40. This figure shows the contribution of the spatial shift in the average separation between the pion and kaon average emission points in the pair rest frame. When the temperature is low $(0.01 \mathrm{GeV})$, both spatial and time separation are small as shown by the dash lines in figures 40 and 41 As the temperature increases, the pion emission time increases faster than the kaon emission time; the higher the temperature the 


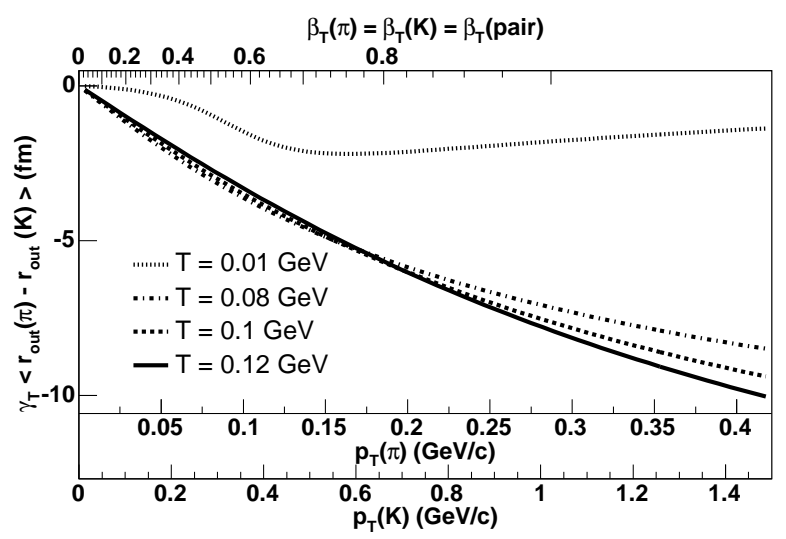

FIG. 40: Contribution of the spatial shift to the average separaration between pions and kaons in the pair rest frame as a function of the pion and kaon momentum for different temperature. The values of the other blast-wave parameters are for a round source, as listed in Table \

larger the shift between pion and kaon average emission time (after boosting into the pair rest frame). On the other hand the spatial shift varies little within the temperature range expected in relativistics heavy-ion collisions (0.08-0.12 GeV). At temperature above $10 \mathrm{MeV} / \mathrm{c}$, a fraction of the pion and kaon sources is truncated even at low particle velocity. The fraction of the source that is truncated, which leads to an inward shift of the average emission radius, varies with transverse momentum and particle mass but it is relatively insensitive to temperature variation between 0.08 and $0.12 \mathrm{GeV}$. It is interesting to note that the average spatial separation between pion and kaon in the laboratory frame actually decreases as the particle velocity rises above $0.8 \mathrm{c}$. However, this decrease is not visible in Figure 39 and 40 becayse the $\gamma_{T}$ factor applied when boosting to the pair rest frame rises faster with velocity than the separation between pions and kaons in the laboratory frame decreases.

Changing the maximum flow rapidity $\left(\rho_{0}\right)$ also affects the separation between pions and kaons in the pair rest frame as shown in Figure 42 When $\rho_{0}=0$, pions and kaons are emitted from the same space point as shown on Figure 43 and only the time shift remains (Figure 44). Indeed, the time shift depends weakly on $\rho_{0}$. Figure 44 shows that the contribution of the time shift to the separaration between pions and kaons in the pair rest frame reaches a plateau when the pion momentum reaches 0.25 $\mathrm{GeV} / \mathrm{c}$. The magnitude of the time shift starts decreasing in the laboratory frame upon reaching this pion momentum but it is compensated by an increase of the boost factor $\gamma_{T} \beta_{T}$. On the other hand, when $\rho_{0}$ is large enough, a significant spatial separation appears in the pair rest frame, which is sensitive to the value of $\rho_{0}$. The main effect of increasing the flow strength is to decrease the pion (and kaon) transverse source size, as shown in Figure 31] In the laboratory frame, the spatial shift between pion and kaon average emission point switches from decreas-

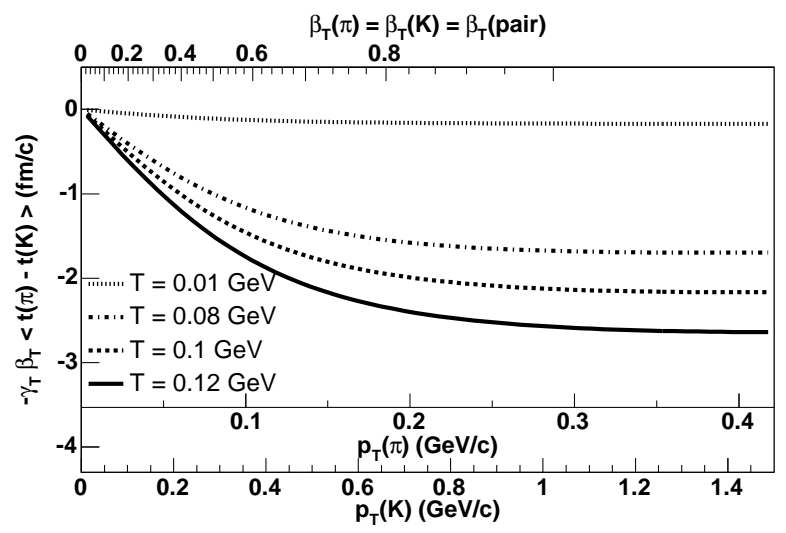

FIG. 41: Contribution of the time shift to the average separaration between pions and kaons in the pair rest frame as a function of the pion and kaon momentum for different temperature. The values of the other blast-wave parameters are for a round source, as listed in Table 【

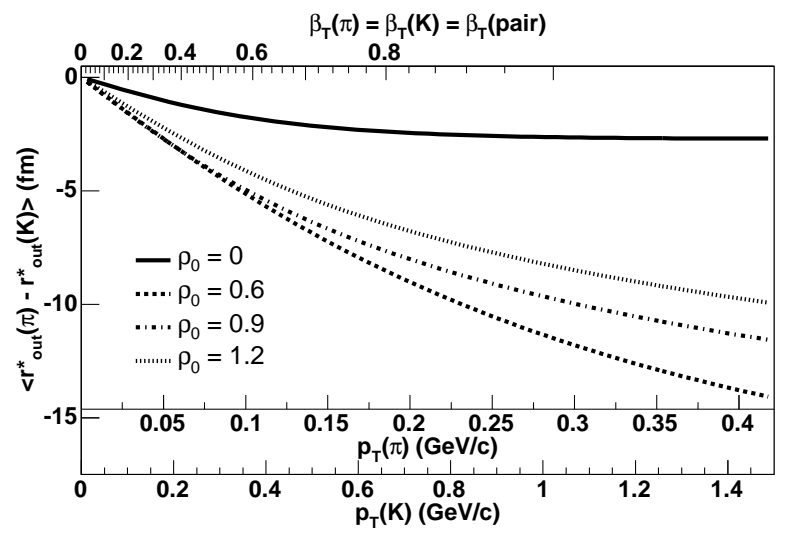

FIG. 42: Average shift between the pion and kaon emission point in the pair rest frame as a function of the pion and kaon momentum for different flow rapidity $\left(\rho_{0}\right)$ The values of the other blast-wave parameters are for a round source, as listed in Table 【

ing to increasing as the particle velocity increase. The value of the velocity where this switch occurs, depends on the value of $\rho_{0}$. The increase of the spatial shift between pions and kaons arises from two effects: the first is the expected shift of the average emission point of both particles due to the flow profile; the second is that the kaon source size drops more rapidly than the pion source size. Then, above a velocity that depends on $\rho_{0}$, the fraction of pion that would be emitted beyond the system limit starts dropping faster than the corresponding fraction of kaons, thus the separation between the average emission points of pions and kaons decreases. However, this turn over, which takes place in the laboratory frame is not directly visible in Figure 43 because the boost factor $\gamma_{T}$ compensates it.

Figure 45 shows the sensitivity of the average separation between pion and kaon emission point in the pair 


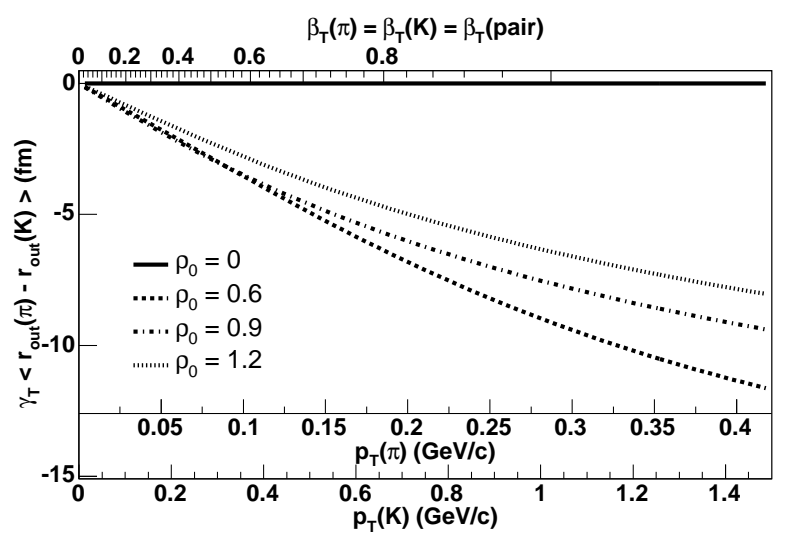

FIG. 43: Contribution of the spatial shift to the average separaration between pions and kaons in the pair rest frame as a function of the pion and kaon momentum for different flow rapidity $\left(\rho_{0}\right)$ The values of the other blast-wave parameters are for a round source, as listed in Table I

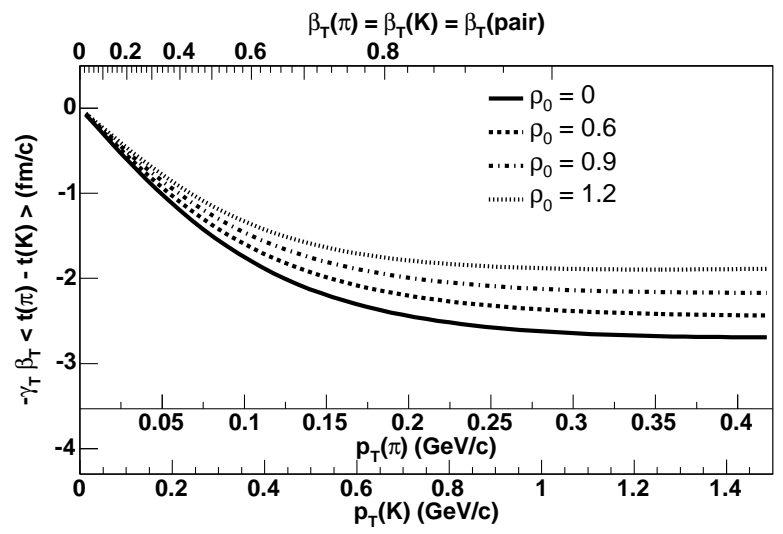

FIG. 44: Contribution of the time shift to the average separaration between pions and kaons in the pair rest frame as a function of the pion and kaon momentum for different flow rapidity $\left(\rho_{0}\right)$ The values of the other blast-wave parameters are for a round source, as listed in Table

rest frame to varying the system radius. This spatial separation scales directly with the system radius because it does not modify the fraction of pions or kaons that are truncated due to the system finite size.

Like the system radius, the proper life time $\tau_{0}$ acts as a scale driving the shift between pion and kaon emission time. Figure 46] shows the effect of varying $\tau_{0}$ on the separation between pion and kaon in the pair rest frame. The effect of varying $\tau_{0}$ is small because the contribution of the time shift to the separation in the pair rest frame is significantly smaller than the contribution of the spatial separation.

Figure 47 shows the effect of varying $a_{s}$ on the separation between pion and kaon in the pair rest frame. Unlike in Figure 34 $\rho_{0}$ was kept constant. Indeed varying $\rho_{0}$ significantly affects the average emission radii, which hides the effect of changing $a_{s}$ at low velocity. However,

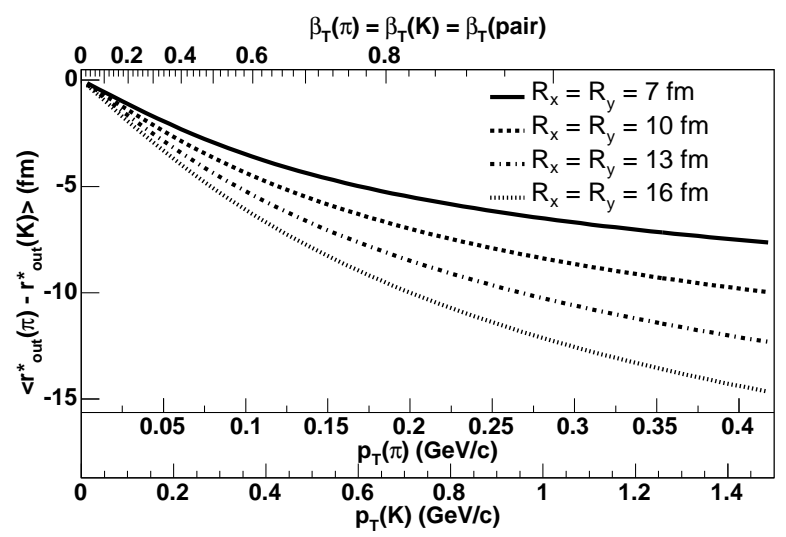

FIG. 45: Average shift between the pion and kaon emission point in the pair rest frame as a function of the pion and kaon momentum for different system radius $\left(R_{x}=R_{y}\right)$. The values of the other blast-wave parameters are for a round source, as listed in Table \

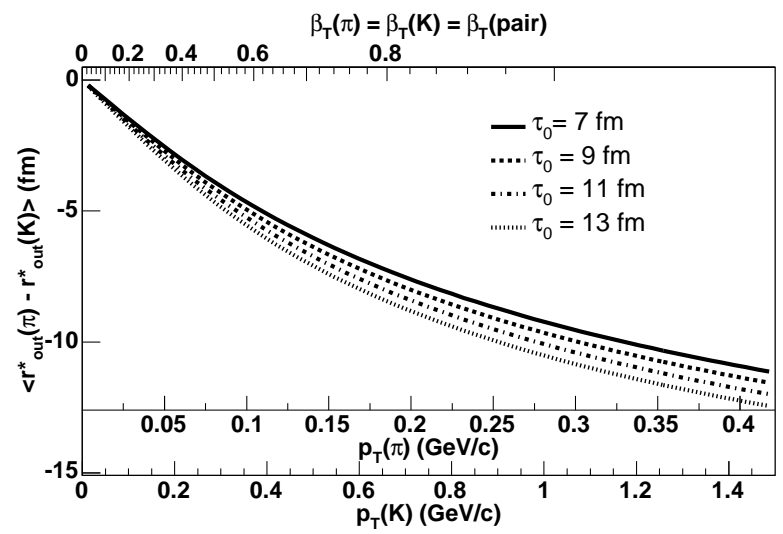

FIG. 46: Average shift between the pion and kaon emission point in the pair rest frame as a function of the pion and kaon momentum for different system proper life time $\left(\tau_{0}\right)$. The values of the other blast-wave parameters are for a round source, as listed in Table 【

when the velocity is larger than $\tanh \left(\rho_{0}\right)=0.716 c$, the amount of boost and space-momentum correlation than particle acquire depends on $a_{s}$; the larger $a_{s}$ the larger the boost. Thus, when the pair velocity is lower than $0.716 c$, the separation decreases with increasing $a_{s}$ because the fraction of truncated particles decrease. When the pair velocity is larger than $0.716 c$, increasing $a_{s}$ has the same consequence as increasing $\rho_{0}$.

\section{Non-identical particle correlations in non-central collisions}

As reported in ref [71], the average space-time separation between different particle species may depend on the particle emission angle with respect to the reaction plane. The effect in the blast-wave parameterization is 


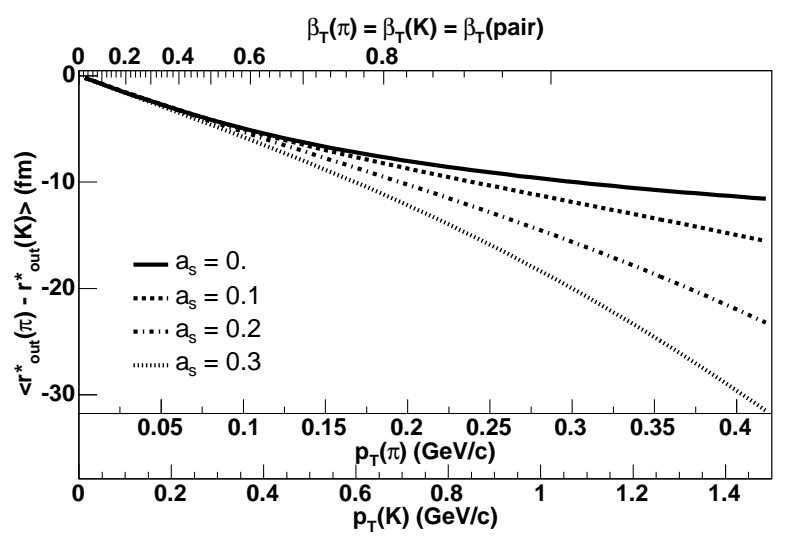

FIG. 47: Average shift between the pion and kaon emission point in the pair rest frame as a function of the pion and kaon momentum for surface smoothness $\left(a_{s}\right)$. The values of the other blast-wave parameters are for a round source, as listed in Table \

shown in Figure 48 The blast-wave parameters are the same as in Figure 35] with the exception of $R_{x}$ and $\rho_{2}$ which are varied. Clear oscillations of $\left\langle r_{\text {out }}^{*}(\pi)-r_{\text {out }}^{*}(K)\right\rangle$ are found when $R_{x}$ is set to $11 \mathrm{fm}$, i.e. when the source is out-of-plane extended $\left(R_{y}=13 \mathrm{fm}\right)$. Small oscillations of $\left\langle r_{\text {side }}^{*}(\pi)-r_{\text {side }}^{*}(K)\right\rangle$ appear as well. The oscillations in both directions are on the order of 1-2 fm, which may be measurable. On the other hand, keeping the source cylindrical but setting the flow modulation parameter $\rho_{2}$ to 0.05 yields very small oscillations, which will be very challenging to probe experimentally. Thus, non-identical two particle correlation analyses with respect to the reaction plane, as pion HBT may provide a good handle on the source shape but not on the flow modulation.

\section{E. Summary of effects of parameters}

Above, we have explored the sensitivity of several experimental observables on various freezeout parameters. Here, we summarize in an orthogonal manner- describing briefly the main observable effects due to an increase in each parameter, if the other parameters are left fixed.

The temperature parameter $T$ quantifies the randomly-oriented kinetic energy component of the freezeout scenario. Increasing this energy component leads to decreased slopes of $p_{T}$ spectra, especially for light-mass particles. Since random motion destroys space-momentum correlations, increasing $T$ reduces measured elliptic flow $\left(v_{2}\right)$ and increases homogeneity scales, i.e. $R_{\mu, 0}^{2}$. On the other hand, for the range of values considered, increasing temperature increases the average separation in the pair rest frame between pions and heavier particles, e.g. $\left\langle r_{\text {out }}^{*}(K)-r_{\text {out }}^{*}(\pi)\right\rangle$.

The $\phi$-averaged transverse flow strength is quantified in this model by $\rho_{0}$. Increasing this directed energy component decreases slopes of $p_{T}$ spectra, especially

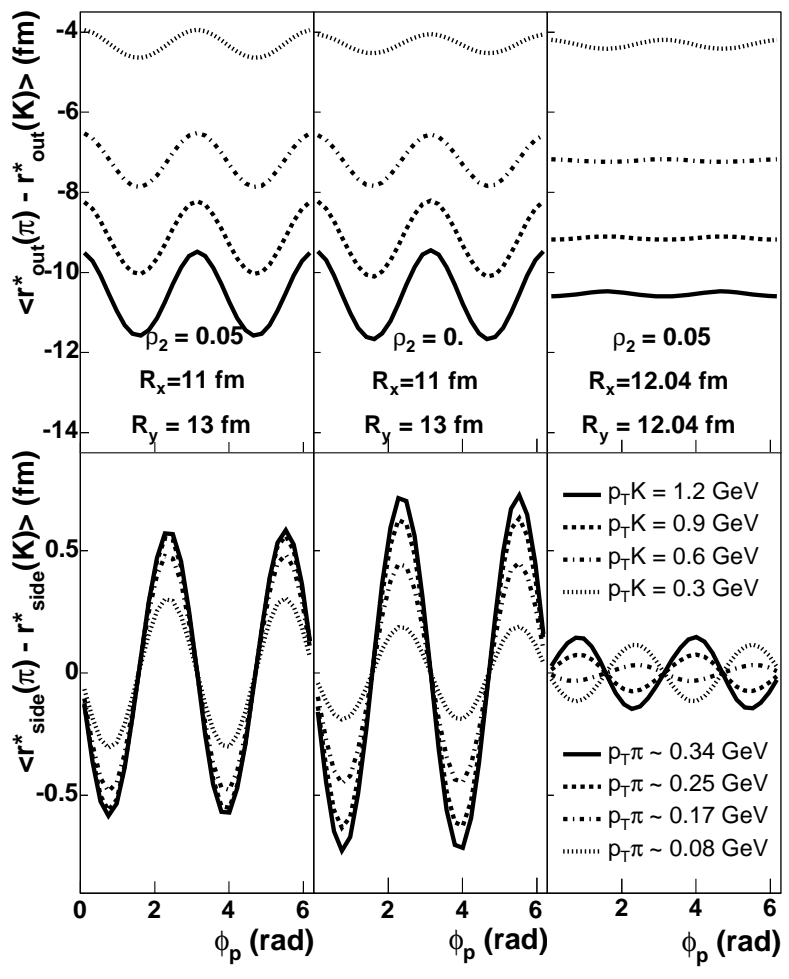

FIG. 48: Average separation between pions and kaons in the pair rest frame as a function of their momentum azimuthal angle with respect to the reaction plane. The four different lines are calculated for 4 different kaon momenta and the corresponding 4 different pion momenta required so that pions and kaons have the same velocity. The values of the blastwave parameters used in the left panel are for a "non-round" source, as listed in Table $\amalg$. The same parameter were used in the middle panel except that $\rho_{2}=0$. The round source parameters are used in the right panel except that $\rho_{2}=0.05$. The separation along the out and side axes are shown on the upper and lower panel respectively.

for heavier particles. Increasing $\rho_{0}$ leads to increasing space-momentum correlations, which reduce $R_{\mu, 0}^{2}$ and $\left\langle r_{\text {out }}^{*}(K)-r_{\text {out }}^{*}(\pi)\right\rangle$, and at constant $\rho_{2}$ reduces $v_{2}$ at high $m_{T}$.

The parameter $a_{s}$ quantifies the "surface diffuseness" of the spatial density profile. Taking care to keep the average transverse flow fixed, variation in $a_{s}$ has little effect on purely momentum-space observables: $p_{T}$ spectra and $v_{2}\left(m, p_{T}\right)$. On the other hand, going from a "box profile" $\left(a_{s}=0\right)$ to a pseudo-Gaussian profile $\left(a_{s}=0.3\right)$ increases the "out-to-side" ratio $R_{o, 0}^{2} / R_{s, 0}^{2}$ at higher $p_{T}$, as the homogeneity region is not constrained by hard geometric "emission boundaries." Furthermore, for a fixed average transverse flow (and flow gradient), increasing $a_{s}$ leads to stronger oscillations in the HBT radius parameters. Increasing $a_{s}$ also increases the $\left\langle r_{\text {out }}^{*}(K)-r_{\text {out }}^{*}(\pi)\right\rangle$ when the temperature and particle velocity are such that the pion source size is significantly larger than the kaon's.

Considering variations in the geometric transverse 
scale of the source $\sqrt{R_{x}^{2}+R_{y}^{2}}$, while keeping all else (including $R_{y} / R_{x}$ ) constant, we conclude that the sensitivity is in the HBT radii and the average separation between different particle species. In HBT, the sensitivity to this parameter is through $R_{o, 0}^{2}$ and $R_{s, 0}^{2}$. The spatial shifts that contribute to the separation $\left\langle r_{\text {out }}^{*}(K)-\right.$ $\left.r_{\text {out }}^{*}(\pi)\right\rangle$ in the pair rest frame scales directly with this parameter. Momentum-space observables such as spectral shapes or $v_{2}$ are unaffected.

We turn now to variations in the timescale parametersthe evolution duration $\left(\tau_{0}\right)$ and emission duration $(\Delta \tau)$. The only significant variations are in $R_{l, 0}^{2}$ and $R_{o, 0}^{2}$, and these depend differently on $\tau_{0}$ and $\Delta \tau$, allowing independent probes of the two parameters. The time shifts that contribute to the separation $\left\langle r_{\text {out }}^{*}(K)-r_{\text {out }}^{*}(\pi)\right\rangle$ in the pair rest frame scales directly with these parameters $\left(\tau_{0}\right.$ and/or $\Delta \tau)$.

Non-central collisions may exhibit azimuthally anisotropic geometry and/or dynamics in the freeze-out configuration. These two effects were studied by varying $R_{y} / R_{x}$, and $\rho_{2}$, respectively. Azimuthally-integrated $p_{T}$ spectra were negligibly affected, as were the $\phi_{p}$-averaged values of $\mathrm{HBT}$ radii $\left(R_{\mu, 0}^{2}\right)$. Observables designed to probe anisotropy, $v_{2}$ and $R_{\mu, 2}^{2}$ of course showed strong sensitivity to geometric or dynamical anisotropy, increasing strongly in magnitude as $R_{y} / R_{x}$ deviates from unity and/or $\rho_{2}$ from zero.

\section{COMPARISON WITH EXISTING DATA}

In the previous section, we reviewed the various experimental observables that the blast-wave parameterization may be able to reproduce. The effect of changing blastwave parameters has been examined. In this section, we investigate how well those parameters can be constrained by the experimental data.

Before comparing the blast-wave parameterization with data, we highlight its limitations and possibly not valid assumptions:

- Freeze-out from a thermalized system at a constant temperature. In the blast wave framework, freezeout is assumed to take place at the same temperature for every particle. In principle, this condition may be relaxed by fitting separately the data for each type of particle. (We do not attempt this here.) Perhaps a more important assumption, however, is made in discussing "temperature" at all; it requires system thermalization. If particles are emitted in a non-thermal way our framework may fail to describe the data.

- Longitudinal boost invariance. The blast wave parameterization relies on longitudinal boost invariance. Elliptic flow data $\left(v_{2}\right)$ published by the PHOBOS collaboration clearly show that longitudinal boost invariance is broken for rapidity larger than
1 [77]. Thus the blast wave parameterization can only be applied within $|Y|<1$. This assumption will be validated or ruled out by high precision data.

- No resonances. The observables that constrain the blast wave are calculated for each particle species using their own masses, ignoring the fact that some particle may be decay products of other particles. In fact a large fraction of pions and protons produced at RHIC energy originate from resonance decays. As mentioned in section 3 , pions from $\omega$ meson decay distort the pion source space-time distribution but this effect is limited because the fraction of pions coming from $\omega$ is on the order of $10 \%$. On the other hand, pion spectra and to a lesser extent proton spectra may be significantly distorted by resonance feed-down. Within the blast wave framework however, it was found in [30, 78] that accounting for resonances affects the Blast Wave parameters extracted fit to spectra by no more than $20 \%$. While $20 \%$ is not negligeable, we feel that the gross features of the parameterization are preserved. On the other hand, an investigation of the effect of resonance feed-down on $v_{2}$ remains to be performed.

We focus on data from Au-Au collisions at $\sqrt{s_{N N}}=$ $130 \mathrm{GeV}$ published by the PHENIX and STAR collaborations at RHIC. The measurements used are listed on top of Table To avoid uncertainties between different experiments and to optimize the overlap between centrality bins, we do not use the full set of data published at RHIC. Three centrality bins are available. The approximate overlap between the centrality bins for the different measures may affect the quality of the fits.

The blast-wave parameters are constrained by simultaneously fitting transverse momentum spectra, transverse momentum azimuthal anisotropy $\left(v_{2}\right)$, and pion HBT radii. Bose-Einstein and Fermi-Dirac distribution are used when appropriate using the approximation to the $4^{t} h$ order as in Equation 8 i.e. the value of $n=4$ in the summation of Equation 8 The quality of the fit to each measure is shown by the $\chi^{2}$ per data points listed in Table III. The number of degrees of freedom is not shown because the number of parameters cannot be subtracted from the number of data points independently for each measure. In addition to the 7 blast-wave parameters, there are 8 scaling parameters used to normalize $\pi^{+}, \pi^{-}$, $K^{+}, K^{-}, p, \bar{p}, \Lambda$, and $\bar{\Lambda}$ spectra. These scaling parameters $(A)$ are not minimized by the fitting function but calculated directly. The $\chi^{2}$ for a given spectra is given by:

$$
\chi^{2}=\sum_{i}\left(\frac{d_{i}-A c_{i}}{e_{i}}\right)^{2}
$$

$d_{i}$ is the measured invariant yield in the bin $i . e_{i}$ is the experimental error on $d_{i}$, and $c_{i}$ is the calculated 


\begin{tabular}{|c|c|c|c|}
\hline & Central & Mid-central & Peripheral \\
\hline \multicolumn{4}{|l|}{ data } \\
\hline$\pi, \mathrm{K}, \mathrm{p}$ spectra [79] & $0-5 \%$ & $15-30 \%$ & $60-92 \%$ \\
\hline$\Lambda$ spectra $[80]$ & $0-5 \%$ & $20-35 \%$ & $35-75 \%$ \\
\hline pion radii [67] & $0-12 \%$ & $12-32 \%$ & $32-72 \%$ \\
\hline Elliptic flow [38] & $0-11 \%$ & $11-45 \%$ & $45-85 \%$ \\
\hline \multicolumn{4}{|l|}{$\overline{\chi^{2} /(\# \text { data points })}$} \\
\hline$\pi^{+} \& \pi^{-}$spectra & $7.2 / 10$ & $26.5 / 10$ & $13.0 / 9$ \\
\hline$K^{+} \& K^{-}$spectra & $24.2 / 22$ & $21.4 / 22$ & $10.1 / 10$ \\
\hline$p \& \bar{p}$ spectra & $10.6 / 18$ & $23.2 / 18$ & $28.0 / 12$ \\
\hline$\Lambda \& \bar{\Lambda}$ spectra & $9.5 / 16$ & $12.8 / 16$ & $11.0 / 16$ \\
\hline$\pi v_{2}$ & $14.6 / 12$ & $29.8 / 12$ & $5.2 / 12$ \\
\hline$p v_{2}$ & $1.6 / 3$ & $9.2 / 6$ & $0.8 / 3$ \\
\hline$\pi r_{\text {out }}$ & $1.9 / 6$ & $0.4 / 2$ & $0.4 / 2$ \\
\hline$\pi r_{\text {side }}$ & $2.7 / 6$ & $0.07 / 2$ & $0.06 / 2$ \\
\hline$\pi r_{\text {long }}$ & $5.3 / 6$ & $0.003 / 2$ & $0.1 / 2$ \\
\hline Total & $77.6 / 99$ & $107.7 / 90$ & $68.7 / 68$ \\
\hline \multicolumn{4}{|l|}{ parameters } \\
\hline $\mathrm{T}(\mathrm{MeV})$ & $106 \pm 3$ & $107 \pm 2$ & $100 \pm 5$ \\
\hline$\rho_{0}$ & $0.89 \pm 0.02$ & $0.85 \pm 0.01$ & $0.79 \pm 0.02$ \\
\hline$\left\langle\beta_{T}\right\rangle$ & $0.52 \pm 0.01$ & $0.50 \pm 0.01$ & $0.47 \pm 0.01$ \\
\hline$\rho_{2}$ & $0.060 \pm 0.008$ & $0.058 \pm 0.005$ & $0.05 \pm 0.01$ \\
\hline$R_{x}(f m)$ & $13.2 \pm 0.3$ & $10.4 \pm 0.4$ & $8.00 \pm 0.4$ \\
\hline$R_{y}(f m)$ & $13.0 \pm 0.3$ & $11.8 \pm 0.4$ & $10.1 \pm 0.4$ \\
\hline$\tau(f m / c)$ & $9.2 \pm 0.4$ & $7.7 \pm 0.9$ & $6.5 \pm 0.6$ \\
\hline$\Delta t(f m / c)$ & $0.003 \pm 1.3$ & $0.06 \pm 1.3$ & $0.6 \pm 1.8$ \\
\hline
\end{tabular}

TABLE II: Upper section: data used in the fit. Middle section: number of $\chi^{2} /$ data points for each measure. Lower section: best fit parameters. Note that $\left\langle\beta_{T}\right\rangle$ is not a fit parameter, but it is calculated from $\rho_{0}$.

invariant yield. The $\chi^{2}$ minimum occurs when $d \chi^{2} / d A$ $=0$, hence:

$$
A=\frac{\sum_{i} d_{i} c_{i} / e_{i}^{2}}{\sum_{i} c_{i} / e_{i}^{2}}
$$

To avoid the region where resonance feed-down may be large and where the Boltzmann approximation may not be valid, fits to transverse momentum spectra are restricted to pions with $p_{T}>0.4 \mathrm{GeV} / \mathrm{c}$. We further restrict the fit range to $p_{T}<1 \mathrm{GeV} / \mathrm{c}$ for pions, $p_{T}<1.5$ $\mathrm{GeV} / \mathrm{c}$ for kaons, $p_{T}<2 \mathrm{GeV} / \mathrm{c}$ for protons and lambdas, because hard processes may significantly contribute to the particle yield above these momenta [28].

Given the large number of data points from spectra, the temperature $(\mathrm{T})$ and the maximum flow rapidity $\left(\rho_{0}\right)$ parameters are constrained by transverse mass spectra. A comparison of data and the blast-wave calculation with the best fit parameters is shown in Figure 49 In semilogarithmic plots, the data and the model fits seem to agree very well. The $\chi^{2} /$ (\# data points) in Table II show, however, that the fit quality varies between particle species and centrality bins. The correlation between the

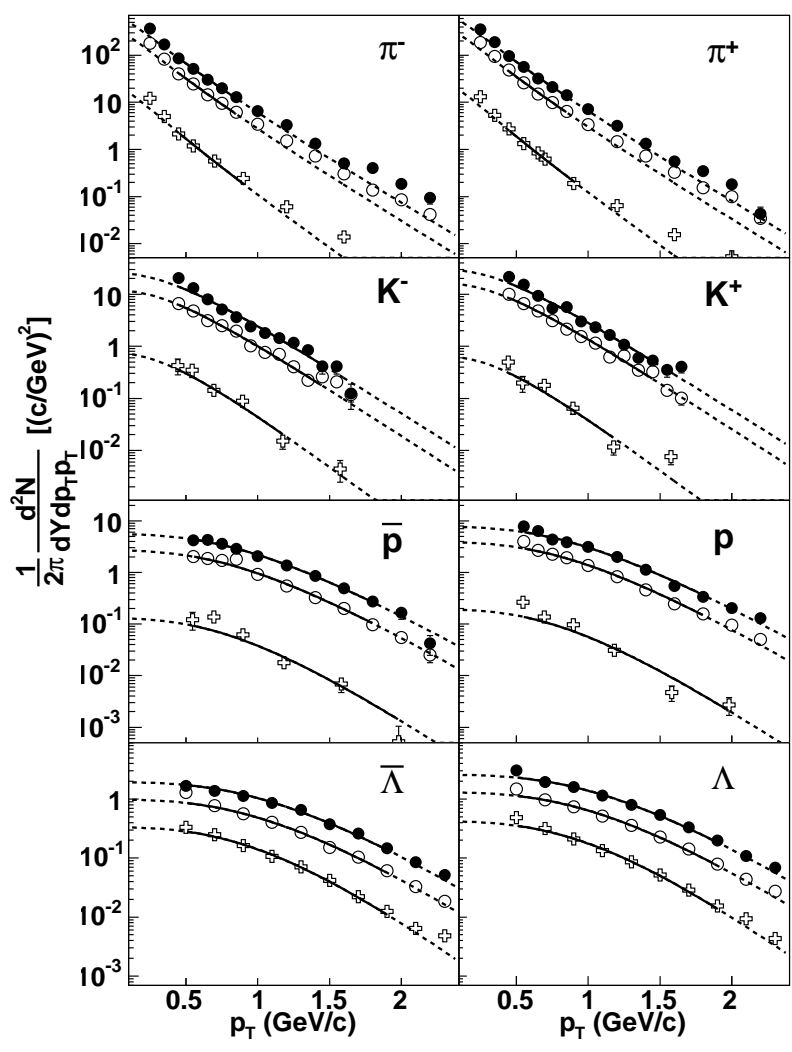

FIG. 49: Comparison of the data with the blast-wave calculations performed with the best fit parameters in three centrality bins. The closed circles are central data, the open circles are mid-central data and the crosses are peripheral data. The plain lines show the blast wave calculation within the fit range while the dash lines show the extrapolation over the whole range.

flow rapidity and temperature is shown in Figure 50, The extracted temperatures and flow velocities are consistent with similar studies reported in Ref. [29, 30, 32, 81]. The differences between these analyses are due to the use of different data sets, different fit ranges, and different flow and spatial profiles. The study reported in Ref. [30] shows that accounting for resonance feed-down leads to a temperature $30 \%$ higher and a transverse velocity $15 \%$ lower than in our study.

The fit of $v_{2}$ mainly constrains the flow rapidity modulation parameter, $\rho_{2}$, and the ratio between the radius inplane and out-of-plane, $R_{x} / R_{y}$. A comparison between the data and the blast-wave calculation with the best fit parameters is shown in Figure [51] The correlation between $\rho_{2}$ and $R_{x} / R_{y}$ is shown in Figure 52. The fit performed in this paper differs from the fit reported together with the data in Ref. 38|. There are three main differences: (i) in Ref. [38], the temperature and flow velocity are free parameters while in this paper they are mostly determined by spectra shapes, (ii) a filled volume and a flow profile are used instead of a shell, (iii) the fits are performed on three centrality bins instead of 


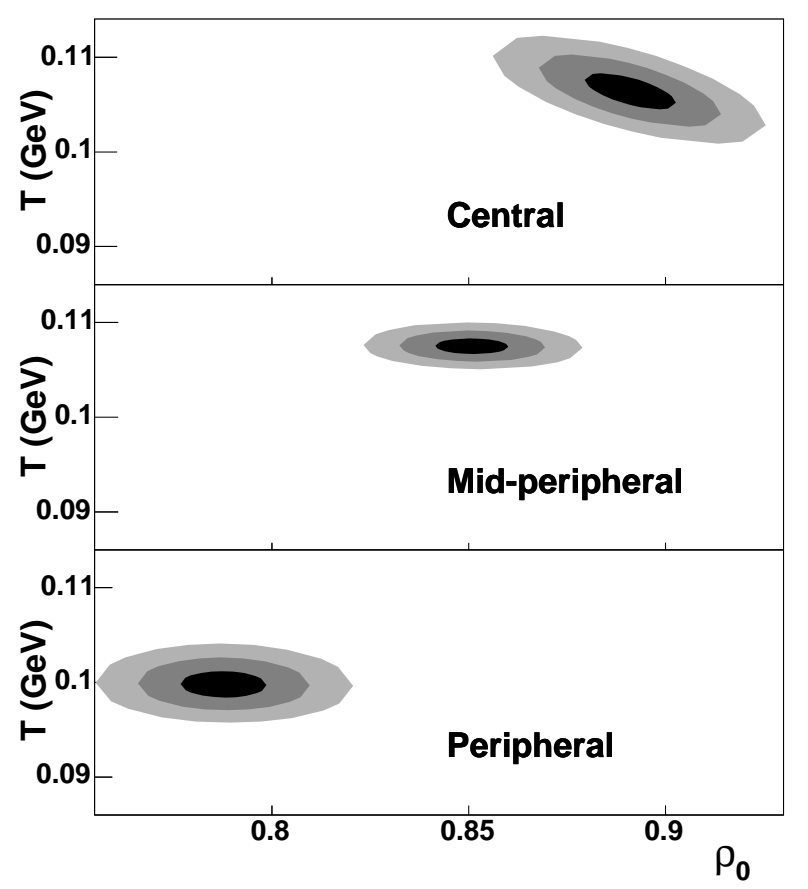

FIG. 50: Number of $\sigma$ contour of the maximum flow rapidity $\left(\rho_{0}\right)$ with respect to the temperature $(\mathrm{T})$. The shadows show the 1, 2 and $3 \sigma$ contours from darkest to lightest.

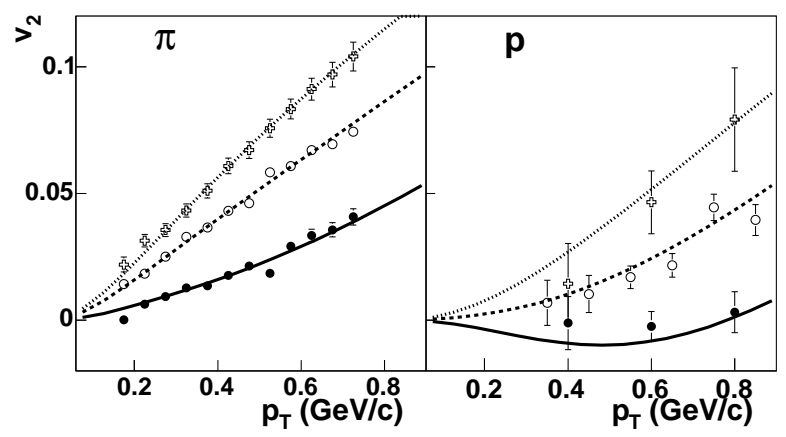

FIG. 51: Comparison of the $v_{2}$ data with the blast-wave calculations performed with the best fit parameters in three centrality bins. The closed circles are central data, the open circles are mid-central data and the crosses are peripheral data.

the minimum bias sample. Thus, a direct comparison between the fit parameters extracted in this paper and those reported in Ref. 38] is difficult. The freeze-out spatial anisotropy probed by the ratio $R_{x} / R_{y}$, increases when increasing the initial state spatial anisotropy, i.e. when going from central to peripheral events. The flow modulation decreases from central to peripheral events, following the same trend as $\rho_{0}$.

The pion source radii allow us to estimate the parameters $R_{x}$ (or $R_{y}$ ), $\tau$ and $\Delta t$. As discussed earlier, the ratio $R_{x} / R_{y}$ is constrained by the fit to $v_{2}$. In the mid-peripheral and peripheral bins, the pion source size

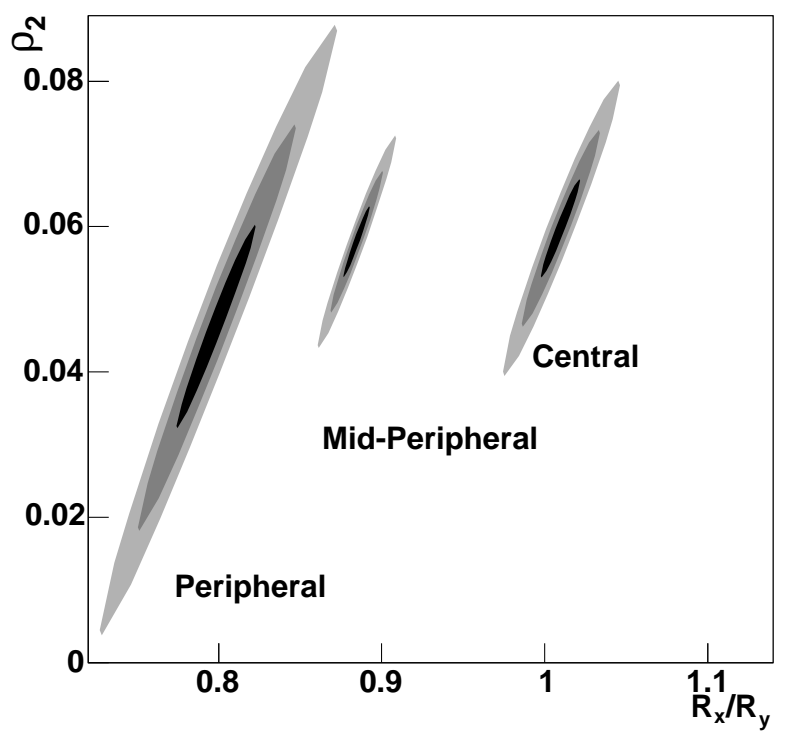

FIG. 52: Number of $\sigma$ contours of the ratio of the in-plane radius over the out-of-plane radius $\left(R_{x} / R_{y}\right)$ with respect to the flow rapidity oscillation parameter $\left(\rho_{2}\right)$. The colors show the 1,2 and $3 \sigma$ contours from darkest to lightest.

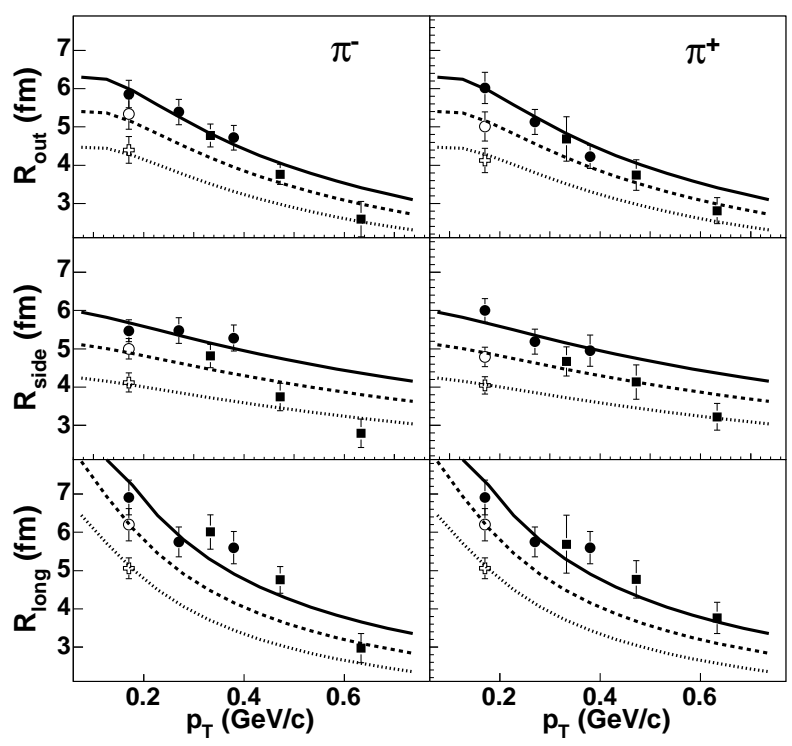

FIG. 53: Comparison of the pion source data measured by the STAR (circle) and the PHENIX (box) collaborations with the blast-wave calculations. Only the STAR data were used to constrain the blast-wave parameters. The closed circles are the central data, the open circles are the mid-central data and the crosses are peripheral data.

has been measured at only one transverse momentum, which weakly constrain the parameters. The comparison between the data and the blast-wave calculation performed with the best fit parameters is shown in Figure 53 This figure includes the pion source radii reported by the PHENIX collaboration [51] although they weren't 
used in the fit. These radii were measured over the $30 \%$ most central events, which doesn't overlap well with the STAR collaboration centrality bins (0-12\%, 12-32\% and 32-72\%). Qualitatively the PHENIX data points agree with the blast-wave fit as they oscillate between the central and mid-peripheral lines. The $\chi^{2}$ obtained from the fit is small, because the systematic errors were added in quadrature to the statistical errors. The system radius increases from peripheral events to central events following the growth of the system initial state. The final system radius is roughly twice the initial system radius, which is a clear evidence of the system expansion. The proper time $(\tau)$ is surprisingly small. Typically, hydrodynamical calculations reach kinetic freeze-out in $15 \mathrm{fm} / \mathrm{c}$, not $9 \mathrm{fm} / \mathrm{c} 82$. The hydrodynamical calculations may be over-predicting the system life-time or the assumption underlying the extraction of $\tau$ may not be valid. The assumption of a boost invariant expansion along the beam axis from a narrow (less than $1 \mathrm{fm}$ thick) pancake may be too simplistic. Indeed, it has been shown that accounting for the fluid viscosity breaks the boost invariant flow 25]. Furthermore, three dimensional hydrodynamical calculations reproduce the measured pseudo-rapidity distribution only when the initial width of the system along the beam axis extends over several units of spatial pseudo-rapidity 83]. Such initial conditions lead to a longitudinal expansion that also breaks boost invariant scaling. In these calculations, even though the system persists for $15 \mathrm{fm} / \mathrm{c}$, the $R_{\text {long }}$ radii is well reproduced. Thus, the absolute value of proper life-time, $\tau$, extracted within the blast-wave parameterization may be questionable. Along the same lines, the very short emission duration is surprising. Indeed, a scenario where particles are emitted in a flash would appear rather unlikely. (On the other hand, processes such as supercooling [84] have been discussed, and may lead to such a scenario.) Recent experimental data [85] show that the value of the $R_{\text {out }}$ radius may have been underestimated by the experiments. Thus, since the emission duration parameters $\Delta t$ strongly depends on $R_{\text {out }}$, it is not appropriate to draw definite conclusions until the experimental issues are settled.

We have shown that $\pi, \mathrm{K}, \mathrm{p}, \Lambda$ transverse momentum spectra, $\pi$, p elliptic flow, and pion source radii measured in $\mathrm{Au}-\mathrm{Au}$ collisions at $\sqrt{s_{N N}}=130 \mathrm{GeV}$ are well reproduced by the blast-wave parameterization. With the exception of the time-scale parameters (system proper life time and emission duration), which fall short of any realistic model calculations, the fit parameters are within expectations. This issue may be resolved when new data on pion source radii become available. Based on the published data in $\mathrm{Au}-\mathrm{Au}$ collisions at $\sqrt{s_{N N}}=130 \mathrm{GeV}$, we conclude that the blast-wave parameterization provides a good description of the system freeze-out stage.

This conclusion will be tested in the future, using the sensitivity of the blast-wave parameterization to observables that have been presented in the previous section: the oscillation of the pion radii with respect to the re-

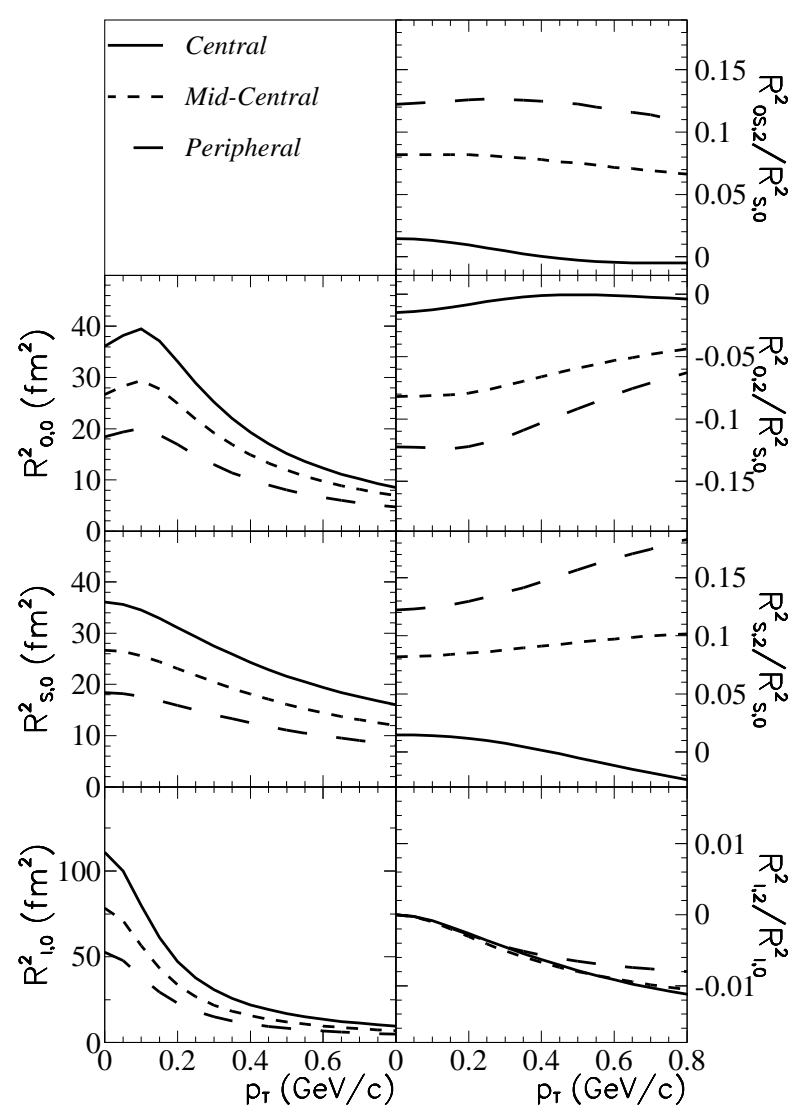

FIG. 54: Oscillations of the pion source radii obtained with the best fit parameters in the three centrality bins.

action plane, and the space-time separation between the emission points of different particle species. Using the parameters extracted from the fits we have calculated the corresponding oscillation of the pion radii with respect to the reaction plane (Figure [54) and the separations between the average space-time emission point of pions kaons and protons (Figure [55). We have also calculated the kaon source radii as shown in Figure 56 since they may become available from the RHIC experiments [85, 86]. The blast-wave parameterization faces the challenge of simultaneously reproducing a large variety of observables that will be measured in Au-Au collisions at $200 \mathrm{GeV}$, namely: (1) pion, kaon, proton and $\Lambda$ transverse momentum spectra, (2) the elliptic flow of many particle species, (3) the pion source radii including the oscillations with respect to the reaction plane, (4) the kaon source radii, (5) the space-time separation between pion, kaon and proton sources. If a satisfactory agreement between these various measurements is achieved, it would provide evidence of a collective expansion that would be very challenging to avoid. Furthermore, when high quality data becomes available that allows for separate fits to each particle species, the blast-wave parameterization may prove to be an important and handy tool 


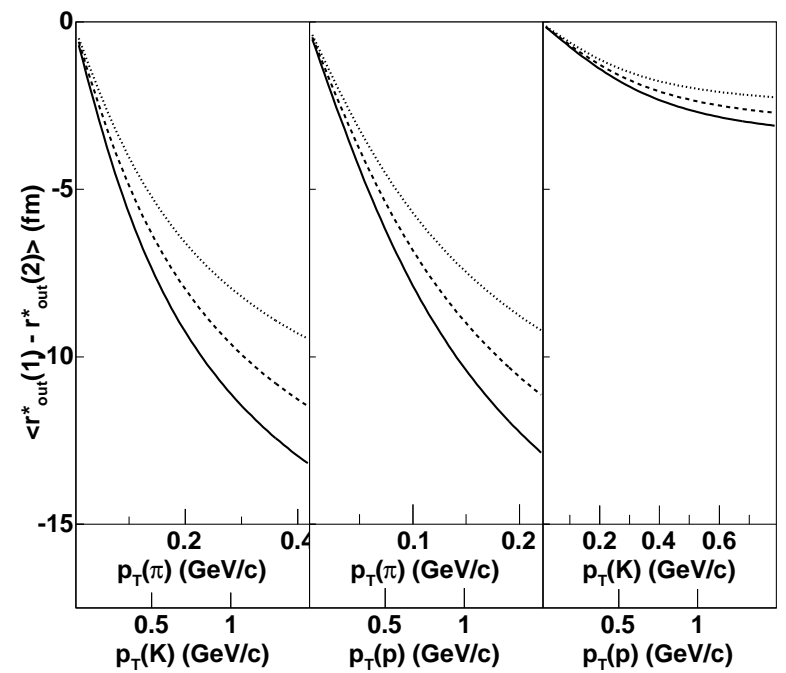

FIG. 55: Space-time separation between pions and kaons (left), pions and protons (middle) and kaons and protons (right) calculated with the best fit parameters in the three centrality bins. Plain line: parameters from fit to central data. Dash line: parameters from mid-peripheral data. Dotted line: parameters from fit to peripheral data.

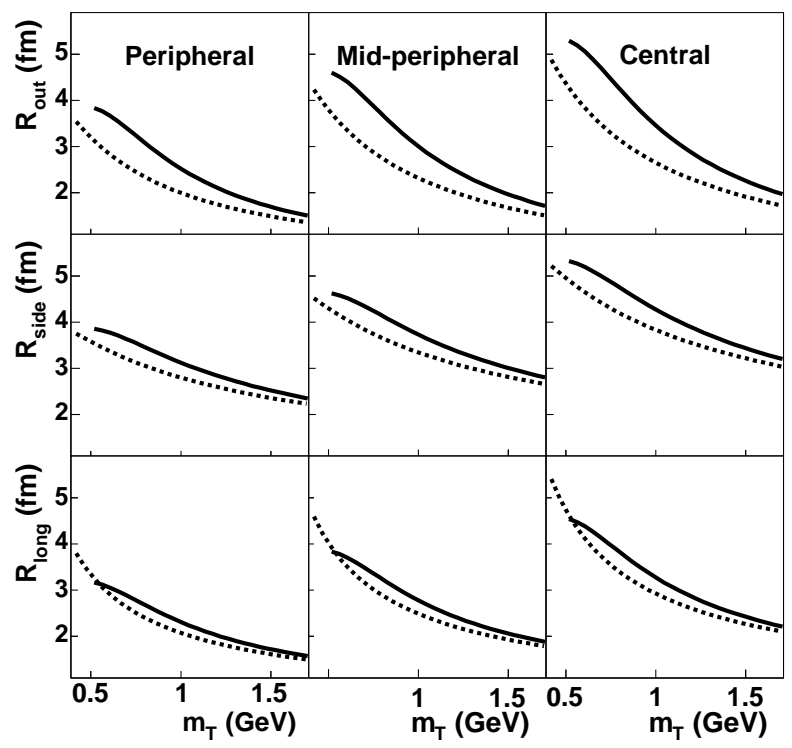

FIG. 56: Kaon source radii (plain line) compare to the pion radii (dash line). The blast-wave calculation are performed with the best fit parameters in each centrality bins.

to assess whether or not the freeze-out time depends on the hadronic cross-sections. It will be especially interesting to study particles with presumably low hadronic cross sections such as the $\phi, \Xi, \Omega$. Since these particles may not be sensitive to hadronic collectivity, they may have a higher freeze-out temperature, they may pick up less collective flow, and freeze-out earlier and from a smaller system than $\pi, \mathrm{K}, \mathrm{p}, \Lambda$. It may be possible to distinguish between the collective expansion at the partonic (if any) versus hadronic stage.

\section{SUMMARY AND OUTLOOK}

We have discussed a scenario of particle kinetic freezeout in heavy ion collisions, inspired by the results of full hydrodynamic calculations of the collision evolution. The scenario, while simplified, nevertheless includes several important features which drive experimental observables of the bulk properties of the system. This includes random ("thermal") motion superimposed upon collective flow fields, an anisotropic transverse geometry, and a freeze-out distribution in proper time. The main assumptions of the model are: longitudinal boost-invariance; the same parametric source for all particle types (e.g. $\pi, p$ ); and the invariance of source parameters over the kinetic freeze-out process (or, equivalently, that the model source parameters can represent the appropriate average of the time-evolving parameter). We discussed the interplay of the various features, such as space-momentum correlations depending on the competition between thermal and collective motion and the source geometry.

The various features (e.g. random thermal motion) were quantified with several parameters (e.g. $T$ ) in a mathematical model outlined in Section 2. The general class of integrals which relate the model emission function to the various experimental observables was identified.

The sensitivity of several observables to the underlying physics represented by the parameters was studied by systematically varying each parameter in Section 3 . In addition to momentum-space observables such as the $p_{T}$ spectra and elliptic flow, which have been discussed previously in the context of similar models, we focussed on the sensitivity of final-state correlations between nonidentical particles and azimuthally-sensitive HBT, new coordinate-space-sensitive analysis tools now becoming available with the quality high statistics datasets from RHIC.

The non-trivial interplay between competing physics effects was different for the different observables. It is clear that the model parameter-space can only be constrained by examining several observables simultaneously; e.g. $p_{T}$ spectra alone are insensitive to the source shape $\left(R_{y} / R_{x}\right)$ and only crudely distinguish between high transverse flow and high temperature. Furthermore, from Section 3, it is clear that experimental data can over-constrain the model parameter space. If consistency with $p_{T}$ spectra and HBT data demand a high temperature and small transverse boost velocity, this has inescapable consequences for $\pi-K$ correlations and elliptic flow. This allows for the "break-down" of the parametric model, and indicates the need to consider new driving physics effects.

In Section 4, the model was used as a functional form in a simultaneous fit to particle spectra $\left(\frac{d N}{d p_{T}}\left(p_{T}, m\right)\right)$, elliptic flow $\left(v_{2}\left(p_{T}, m\right)\right)$, and pion $\operatorname{HBT}\left(R\left(p_{T}\right)\right)$ for three centrality classes from published $\mathrm{RHIC}$ results for $\mathrm{Au}-\mathrm{Au}$ 
collisions at $\sqrt{s_{N N}}=130 \mathrm{GeV}$. While higher- $p_{T}$ HBT radii from PHENIX are only qualitatively described, a good fit is obtained to the $p_{T}$ spectra, elliptic flow, and low- $p_{T}$ STAR HBT radii.

Most of the physical parameter values obtained from the fits, as well as their evolution with collision centrality, fall within reasonable expectations. The exceptions are the evolution duration $\tau_{0}$ and emission duration $\Delta \tau$, which are shorter than most physical models predict. The $\Delta \tau$ discrepancy may be partially resolved by an improved experimental treatment of the Coulomb interaction in HBT analyses; this will be evaluated upon publication of the new results from RHIC. The $\tau_{0}$ value is strongly connected to the model assumption of boostinvariance; if this assumption is invalid at mid-rapidity at RHIC (e.g. if there is longitudinal acceleration in the early system evolution), we expect our fit values to be only lower limits on the evolution duration.

Finally, Section 4 discusses expectations for kaon HBT, azimuthally-sensitive pion HBT, and emission space-time separations between non-identical particles $(\pi, K, p)$, based on the fit parameters to the published data. Expectations for the azimuthally-sensitive pion HBT are driven by the elliptic flow data, which indicates $R_{y}>R_{x}$ (see Table III)- i.e. the freeze-out system shape is outof-plane extended, qualitatively similar to the geometrical overlap configuration in the entrance channel of the collision. From Section IIIC it is clear that this determines the phases (and amplitudes) of the expected oscillations in the HBT radii- i.e. the signs and magnitudes of $R_{\mu, 2}^{2}$. If azimuthally-sensitive HBT measurements confirm this out-of-plane freeze-out configuration, it might provide further evidence of short evolution times $\tau_{0}$, since it would imply that the source, which is expanding faster in the reaction plane than out of it, did not have sufficient time to overcome its initial geometric anisotropy before freeze-out. Quantifying the timescale in this way is outside the scope of the blast-wave model, which does not attempt to describe the system evolution.

Exploration of simple parameterizations such as the blast-wave is driven by the desire to connect observations to driving underlying physics. Furthermore, a quantita- tive interpretation of many measurements (perhaps especially two-particle interferometry) requires some model assumptions. Naturally, one prefers assumptions which are consistent with other measurements (e.g. elliptic flow) in the same system. Especially since first-principle model calculations have difficulty consistently explaining the range of observations in the soft sector of RHIC, it is hoped that insight may be gained from rather simple theory-inspired parameterizations such as the blast-wave.

Inasmuch as such parameterizations reproduce observations, there is hope that the driving physics effects have been approximately quantified, and one may compare the "underlying" parameters (e.g. timescales) with firstprinciples calculations in an effort to isolate the cause of the discrepancy between such calculations and observation. On the other hand, when simplistic parameterizations cannot, with any set of parameters, reproduce the main features of the data, it indicates that something additional is driving observations. In parameterizations, features may be implemented and "turned on and off," one at a time, testing for consistency with a range of experimental observations. The insight gained from such studies can then be fed back into more "theoretical" models. It is hoped that new experimental data will further constrain- or break- the simple parameterization, generating insights into the dynamics of heavy ion collisions at RHIC.

\section{Acknowledgments}

We gratefully acknowledge fruitful discussions with Drs. J. Castillo, T. Csörgo, U. Heinz, P. Jacobs, P. Kolb, R. Lednicky, D. Magestro L. Ray, R. Snellings, B. Tomášik, S. Voloshin, R. Wells, and U. Wiedemann. We thank Drs. D. Magestro, K. Schweda and P. Sorensen for a careful reading of an early version of the text. This work supported by the U.S. National Science Foundation under Grant No. PHY-0099476 and by the U.S. Department of Energy under Contract No. DE-AC03$76 \mathrm{SF} 00098$.
[1] For reviews and recent developments, see proceedings of the Quark Matter conferences: QM99 [Nucl. Phys. A661 (1999)]; QM01 [Nucl. Phys. A698 (2002)]; QM02 [Nucl. Phys. A715 (2003)].

[2] E.V. Shuryak, Phys. Lett. B78 150 (1978).

[3] E.V. Shuryak, Sov. J. Nucl. Phys. 28408 (1978); J. Kapusta, P. Lichard, and D. Seibert, Phys. Rev. D44 2774 (1991).

[4] BRAHMS Collaboration, Phys. Rev. Lett. 91072305 (2003); PHENIX Collaboration, Phys. Rev. Lett. 87 052301 (2001); ibid, nucl-ex/0207009; ibid, Phys. Rev. Lett 91072301 (2003); ibid, Phys. Rev. Lett 91072303 (2003); ibid, nucl-ex/0308006; PHOBOS Collaboration,
Phys. Rev. Lett. 91072302 (2003); STAR Collaboration, Phys. Rev. Lett. 89202301 (2002); ibid, Phys. Rev. Lett. 90032301 (2003); ibid, Phys. Rev. Lett. 90082302 (2003); ibid, Phys. Rev. Lett. 91172302 (2003); ibid, Phys. Rev. Lett. 91072304 (2003).

[5] R. Baier, D. Schiff, and B.G. Zakharov, Ann. Rev. Nucl. Part. Sci. 5037 (2000); M. Gyulassy, I. Vitev, X.N. Wang, and B. Zhang, nucl-th/0302077.

[6] U.A. Wiedemann and U. Heinz, Phys. Rept. 319, 145 (1999); U. Heinz and B.V. Jacak, Ann. Rev. Nucl. Part. Sci. 49, 529 (1999).

[7] R. Haburry-Brown and R.Q. Twiss, Nature 17727 (1956) and 1781046 (1960). 
[8] S. Soff, S. Bass, D. Hardtke, S. Panitkin, Nucl. Phys. A715 801 (2003).

[9] U. Heinz and P.F. Kolb, Nucl. Phys. A702 269 (2002); hep-ph/0111075.

[10] K. Morita, S. Muroya, C. Nonaka, and T. Hirano, Phys. Rev. C66 054904 (2002).

[11] T. Hirano and K. Tsuda, Phys. Rev. C66 054905 (2002).

[12] B. Zhang, C.M. Ko, B.A. Li, and Z.W. Lin, Phys. Rev. C61 067901 (2000); Z.W. Lin, S. Pal, C.M. Ko, B.A. Li, and B. Zhang, Phys. Rev. C64 011902 (2001).

[13] Especially after so-called "non-flow" contributions 14] are removed from the experimental data, AMPT requires parton cross-sections $\sigma \lesssim 3 \mathrm{mb}$ to reproduce STAR elliptic flow data [15] and $\sigma \approx 10 \mathrm{mb}$ to reproduce HBT data. We thank Zi-wei Lin for bringing this to our attention.

[14] STAR Collaboration, C. Adler, et al, Phys. Rev. C66 034904 (2002).

[15] Z.W. Lin and C.M. Ko, Phys. Rev. C65 034904 (2002).

[16] Z.W. Lin, C.M. Ko, and S. Pal, Phys. Rev. Lett. 89 152301 (2002).

[17] M. Csanád, T. Csörgö, and B. Lörstad, nucl-th/0310040; M. Csanád, T. Csörgö, B. Lörstad, and A. Ster, Acta. Phys. Polon. B35 191 (2004).

[18] P. Huovinen, P.F. Kolb, U. Heinz, P.V. Ruuskanen, S. Voloshin, Phys. Lett. B503 58 (2001).

[19] H. Sorge, Phys. Rev. Lett. 782309 (1997).

[20] T. Humanic, nucl-th/0203004; ibid, Nucl. Phys. A715 641c (2003).

[21] D. Molnar, M. Gyulassy, Nucl.Phys. A698 379 (2002) and nucl-th/0211017.

[22] S. Bass, B. Mueller, D. Srivastava, Phys.Lett. B551 277 (2003).

[23] U. Heinz and P.F. Kolb, Phys. Lett. B542 216 (2002).

[24] D. Teaney, J. Lauret, E. Shuryak, nucl-th/0110037 (2001).

[25] D. Teaney, nucl-th/0301099.

[26] H.G. Fischer, Nucl. Phys. A715 118c (2003).

[27] Y.V. Kovchegov and K.L. Tuchin, Nucl. Phys. A708 413 (2002). Nucl. Phys. A717 249 (2003).

[28] T. Peitzmann, nucl-th/0303046.

[29] J. M. Burward-Hoy for the PHENIX Collaboration, Nucl.Phys. A715 498 (2003).

[30] T. Peitzmann, Eur.Phys.J. C26 539 (2003).

[31] U. Heinz and S.H.M. Wong, Phys. Rev. C66 014907 (2002).

[32] B. Tomasik, proceedings of 38 Rencontres de Moriond, QCD and hadronic interactions, nucl-th/0304079.

[33] G.D. Westfall, J. Gosset, P.J. Johansen, A.M. Poskanzer, W.G. Meyer, H.H. Gutbrod, A. Sandoval, and R. Stock, Phys. Rev. Lett. 371202 (1976).

[34] J.P. Bondorf, S.I.A. Garpman, and J. Zimanyi, Nucl. Phys. A296 320 (1978).

[35] P.J. Siemens and J.O. Rasmussen, Phys. Rev. Lett. 42 880 (1979).

[36] E. Schnedermann, J. Sollfrank, U. Heinz, Phys. Rev. C48 2462 (1993).

[37] J.D. Bjorken, Phys. Rev. D27 140 (1983).

[38] STAR Collaboration, C. Adler, et al, Phys. Rev. Lett. 87 182301 (2001).

[39] S.A. Voloshin and A.M. Poskanzer, Phys. Lett. B474 27 (2000).

[40] S.A. Voloshin, Nucl. Phys. A715 379c (2003); ibid, nuclth/0202072.

[41] S.A. Voloshin, Phys. Rev. C55 1630 (1997).
[42] STAR Collaboration, F. Retière, et al, nucl-ex/0111013 (2001).

[43] S. Pratt, T. Csörgő, and J. Zimanyi, Phys. Rev. C42 2646 (1990); G. Bertsch, M. Gong, and M. Tohyama, Phys. Rev. C37 1896 (1988).

[44] U.A. Wiedemann, Phys. Rev. C57 266 (1998).

[45] B. Tomášik, U.A. Wiedemann, and U. Heinz, Nucl. Phys. A663 753 (2000); B. Tomášik and U. Heinz, Phys. Rev. C65 031902 (2002).

[46] We thank Sergei Voloshin for correcting an error we had previously made in deriving Eq. 6]

[47] M.A. Lisa, U. Heinz, U.A. Wiedemann, Phys. Lett. B489 287 (2000).

[48] U. Heinz, A. Hummel, M.A. Lisa, U.A. Wiedemann, Phys. Rev. C66 044903 (2002).

[49] E895 Collaboration, M.A. Lisa et al, Phys. Lett. B496 1 (2000).

[50] T. Csörgő, S.V. Akkelin, Y. Hama, B. Lukács, and Yu. M. Sinyukov Phys. Rev. C67 034904 (2003).

[51] Phenix Collaboration, K. Adcox, et al., Phys. Rev. Lett. 88192302 (2002).

[52] A.N. Makhlin and Yu.M. Sinyukov, Z. Phys. C39 69 (1988).

[53] S.V. Akkelin and Yu.M. Sinyukov, Phys. Lett. B356 525 (1995).

[54] M. Herrmann and G.F. Bertsch, Phys. Rev. C51 328 (1995).

[55] U.A. Wiedemann, P. Scotto, U. Heinz, Phys. Rev. C53 918 (1996).

[56] T. Csörgő and B. Lörstad, Phys. Rev. C54 1390 (1996).

[57] Y.-F. Wu, U. Heinz, B. Tomášik, and U. Wiedemann, Eur. Phys. J. C1 599 (1998).

[58] Since particle emission occurs only after the collision begins (i.e. when $\tau>0$ ), the lower limit on the $\tau$ integration in Equations [5] and 16] ought to be zero instead of $-\infty$. This distinction vanishes if $\Delta \tau \ll \tau_{0}$, and the effect is negligible for the range of parameter values explored here.

[59] Yu. M Sinyukov, In Hot Hadronic Matter: Theory and Experiment, ed. J. Rafelski. New York: Plenum, NATO ASI Series B 346:309 (1995).

[60] D. Hardtke and S. Voloshin, Phys.Rev. C61 024905 (2000).

[61] D. Brown and P. Danielewicz, Phys.Rev. C64 014902 (2001). P. Danielewicz, D. A. Brown, M. Heffner, S. Pratt and R. Soltz, nucl-th/0407022.

[62] S. Nickerson, T. Csörgö and D. Kiang, Phys.Rev. C57 3251 (1998) .

[63] U. Wiedemann and U. Heinz, Phys.Rev. C56 3265 (1997).

[64] D. Magestro private communication. Model as described in P. Braun-Munzinger, K. Redlich, J. Stachel, Invited review for Quark Gluon Plasma 3, eds. R. C. Hwa and Xin-Nian Wang, World Scientific Publishing, nuclth/0304013.

[65] T. Csörgő, B. Lörstad, and J. Zimányi, Z. Phys. C71 491 (1996).

[66] D.H. Rischke and M. Gyulassy, Nucl. Phys. A608 479 (1996); D.H. Rischke, Nucl. Phys. A610 88c (1996).

[67] STAR Collaboration, C. Adler, et al, Phys. Rev. Lett. 87 082301 (2001).

[68] P. Kolb, D. Magestro, private communications.

[69] R. Lednický, V.I. Lyuboshitz, B. Erazmus, D. Nouais, Phys. Lett. B 373 (1996) 30. 
[70] R. Lednický, nucl-th/0305027; Proc. CIPPQG'01, nuclth/0112011; Proc. XXXII ISMD, nucl-th/0212089.

[71] S. Voloshin, R. Lednický, S. Panitkin, N. Xu, Phys. Rev. Lett. 79 (1997) 4766.

[72] D. Ardouin et al., Phys.Lett. B 446 (1999) 191, nuclth/0203030.

[73] C. Greiner, P. Koch, H. Stöcker, Phys. Rev. Lett. 581825 (1987); B. Lukács, J. Zimányi and N.L. Balazs, Phys. Lett. B183 27 (1987); M. Gyulassy, Phys. Lett. B286 211 (1992).

[74] F. Retière (STAR collaboration) , Nucl Phys. A $\mathbf{7 1 5}$ (2003) 591c, nucl-ex/0212026.

[75] J. Adams et al. (STAR collaboration), submitted to Phys. Rev. Lett., nucl-ex/0307025.

[76] R. Lednický, Proc. 8th Int. Workshop on Multiparticle Production, Correlations and Fluctuations (1998) 148, nucl-th/0304063, and, R. Lednický, S. Panitkin, and N. $\mathrm{Xu}$, nucl-th/0304062.

[77] B. Back et al., Submitted to Phys. Rev. Lett., nucl- ex/0406021.

[78] J. Adams et al., Phys. Rev. Lett. 92 (2004) 112301

[79] K. Adcox et al., Phys. Rev. Lett. 88242301 (2002).

[80] C. Adler et al., Phys. Rev. Lett. 89092301 (2002).

[81] N. Xu and M. Kaneta, Nucl. Phys. A698 306c (2002).

[82] P. Kolb, Proceedings for the 19th Winter Workshop on Nuclear Dynamics, nucl-th/0304036.

[83] T.Hirano and K. Tsuda, Phys.Rev. C66 041901 (2002).

[84] T. Csörgő and L. P. Csernai, Phys.Lett. B333 494 (1994). hep-ph/9406365.

[85] STAR Collaboration, M. López-Noriega, et al, Nucl. Phys. A715 623c (2003).

[86] PHENIX Collaboration, A. Enokizono, et al, Nucl. Phys. A715 595c (2003).

[87] P. Houvinen, P.F. Kolb, and U. Heinz, Nucl. Phys. A698 475c (2002).

[88] E.V. Shuryak, Phys. Rev. C66 027902 (2002). 